\title{
Asymptotic Spectra of Large (Grid) Graphs with a Uniform Local Structure (Part I): Theory
}

\author{
Andrea Adriani, Davide Bianchi and Stefano Serra-Capizzano
}

\begin{abstract}
We are mainly concerned with sequences of graphs having a grid geometry, with a uniform local structure in a bounded domain $\Omega \subset \mathbb{R}^{d}, d \geq 1$. When $\Omega=[0,1]$, such graphs include the standard Toeplitz graphs and, for $\Omega=[0,1]^{d}$, the considered class includes $d$-level Toeplitz graphs. In the general case, the underlying sequence of adjacency matrices has a canonical eigenvalue distribution, in the Weyl sense, and we show that we can associate to it a symbol $\mathfrak{f}$. The knowledge of the symbol and of its basic analytical features provides many information on the eigenvalue structure, of localization, spectral gap, clustering, and distribution type.

Few generalizations are also considered in connection with the notion of generalized locally Toeplitz sequences and applications are discussed, stemming e.g. from the approximation of differential operators via numerical schemes. Nevertheless, more applications can be taken into account, since the results presented here can be applied as well to study the spectral properties of adjacency matrices and Laplacian operators of general large graphs and networks.
\end{abstract}

Mathematics Subject Classification (2010). 05C50; 05C22; 34B45; 65N22.

Keywords. Large graphs and networks; eigenvalues distribution; graph-Laplacian.

\section{Introduction}

Spectral properties of the adjacency matrix and the Laplacian operator of graphs provide valuable insights regarding a large number of key features such as the Shannon capacity, Chromatic number, diameter, maximum cut, just to cite few of them, see $[6,35]$, which often play a central role in many applied real-world problems e.g. in physics and chemistry problems, see as references $[15,21,34]$ and $[12$, Chapter 8$]$. In particular, graphs typically describe approximations of physical domains related

The authors are supported by INdAM-GNCS Gruppo Nazionale per il Calcolo Scientifico. 
to self-adjoint second order linear differential operators: for example, the discretization of the Laplace differential operator with Dirichlet boundary conditions over a membrane $\Omega \in \mathbb{R}^{d}$ produces the Laplacian matrix of a (possibly infinite) graph with its eigenvalues corresponding to the characteristic frequencies of the membrane, $[12$, p.256]. In the last few years there has been a rising interest over this topic, especially concerning spectral convergence of the graph-Laplacian towards the spectrum of its continuous counterpart, see the seminal work of D. Burago and coauthors [8] and applications in inverse problems regularization and machine learning, refer to $[43,44,45]$. Therefore, having a way to analytically measure the eigenvalue distribution of the adjacency matrix and the graph-Laplacian can be as precious as crucial in many applications.

In this work we are interested in defining and studying a large class of graphs enjoying few structural properties:

a. when we look at them from "far away", they should reconstruct approximately a given domain $\Omega \subset[0,1]^{d}, d \geq 1$, i.e., the larger is the number of the nodes the more accurate is the reconstruction of $\Omega$;

b. when we look at them "locally", that is from a generic internal node, we want that the structure is uniform, i.e., we should be unable to understand where we are in the graphs, except possibly when the considered node is close enough to the boundaries of $\Omega$.

Technically, we are not concerned with a single graph, but with a whole sequence of graphs, where $\Omega$ and the internal structure are fixed, independently of the index (or multi-index) of the graph uniquely related to the cardinality of nodes: thus the resulting sequence of graphs has a grid geometry, with a uniform local structure, in a bounded domain $\Omega \subset \mathbb{R}^{d}, d \geq 1$. We assume the domain $\Omega$ to be Lebesgue measurable with regular boundary, which is for us a boundary $\partial \Omega$ of zero Lebesgue measure, and contained for convenience in the cube $[0,1]^{d}$. We call regular such a domain. When $\Omega=[0,1]$, it is worth observing that such graphs include the standard Toeplitz graphs (see [28] and Definition 4.1) and for $\Omega=[0,1]^{d}$ the considered class includes $d$-level Toeplitz graphs (see Definition 4.2).

The main result is the following: given a sequence of graphs having a grid geometry with a uniform local structure in a domain $\Omega$, the underlying sequence of adjacency matrices has a canonical eigenvalue distribution, in the Weyl sense (see $[5,29]$ and references therein), and we show that we can associate to it a symbol function $\mathfrak{f}$. More precisely, when $\mathfrak{f}$ is smooth enough, if $N$ denotes the size of the adjacency matrix (i.e. the number of nodes of the graph), then the eigenvalues of the adjacency matrix are approximately values of a uniform sampling of $\mathfrak{f}$ in its definition domain, which depends on $\Omega$ (see Definition 2.3 for the formal definition of eigenvalue distribution in the Weyl sense and the results on Section 5 for the precise characterization of $\mathfrak{f}$ and of its definition domain).

The knowledge of the symbol and of some of its basic analytical features provides a lot of information on the eigenvalue structure, of localization, spectral gap, clustering, and distribution type. 
The mathematical tools are mainly taken from the field of Toeplitz (see the rich book by Böttcher and Silbermann [5] and [29, 42, 46]) and Generalized Locally Toeplitz (GLT) matrix-sequences (see [37, 38, 41]): for a recent account on the GLT theory, which is indeed quite related to the present topic, we refer to the following books and reviews $[22,23,24,26]$.

Interestingly enough, as discussed at the end of this paper, many numerical schemes (see e.g. [10, 11, 39]) for approximating partial differential equations (PDEs) and operators lead to sequences of structured matrices which can be written as linear combination of adjacency matrices, associated with the graph sequences described here. More specifically, if the physical domain of the differential operator is $[0,1]^{d}$ (or any $d$-dimensional rectangle) and the coefficients are constant, then we encounter $d$ level (weighted) Toeplitz graphs, when approximating the underlying PDE by using e.g. equispaced Finite Differences or uniform Isogeometric Analysis (IgA). On the other hand, under the same assumptions on the underlying operator, quadrangular and triangular Finite Elements lead to block $d$-level Toeplitz structures, where the size of the blocks is related to the degree of the polynomial space of approximation and to the dimensionality $d$ (see [25]). Finally, in more generality, the GLT case is encountered by using any of the above numerical techniques, also with nonequispaced nodes/triangulations, when dealing either with a general domain $\Omega$ or when the coefficients of the differential operator are not constant. The given classification of approximated PDE matrix-sequences is relevant also from a practical viewpoint since the obtained spectral information can be used for guiding the design of proper iterative solvers (in terms either of preconditioners or of ad hoc multigrid methods) for the underlying linear systems with large matrix size: see [1] for the use of the theoretical results of the current work for the design of preconditioners and of multigrid procedures.

The paper is organized as follows. In Section 2 and Section 3 we collect all the machinery we need for our derivations: we will first review basic definitions and notation from graph theory, from the field of Toeplitz and $d$-level Toeplitz matrices, and then we provide the definitions of canonical spectral distribution, spectral clustering etc. In particular, we introduce Theorem 3.2 which plays a central role for the spectral analysis in applications. In Section 4 we present the structure of $d$-level diamond Toeplitz graphs. In Section 5 we give formal definitions of sequences of graphs having a grid geometry, with a uniform local structure, in regular domains $\Omega \subset[0,1]^{d}, d \geq 1$, and we prove the main results, by identifying the related symbols. Section 6 and 7 contain specific applications, including the analysis of spectral gaps and the study of connections with the numerical approximation of differential operators by local methods, such as Finite Differences, Finite Elements, Isogeometric Analysis etc. Finally, Section 8 is devoted to draw conclusions and to present open problems. 


\section{Background notation and definitions}

In this section we present some definitions, notation, and (spectral) properties associated with graphs (see [12] and references therein) and, in particular, with Toeplitz graphs [28].

Before proceeding further, let us introduce a multi-index notation that we use hereafter. Given an integer $d \geq 1$, a $d$-index $\boldsymbol{k}$ is an element of $\mathbb{Z}^{d}$, that is, $\boldsymbol{k}=$ $\left(k_{1}, \ldots, k_{d}\right)$ with $k_{r} \in \mathbb{Z}$ for every $r=1, \ldots, d$. We intend $\mathbb{Z}$ equipped with the lexicographic ordering, that is, given two $d$-indices $\boldsymbol{i}=\left(i_{1}, \ldots, i_{d}\right), \boldsymbol{j}=\left(j_{1}, \ldots, j_{d}\right)$, we write $\boldsymbol{i} \triangleleft \boldsymbol{j}$ if $i_{r}<j_{r}$ for the first $r=1,2, \ldots, d$ such that $i_{r} \neq j_{r}$. The relations $\unlhd, \triangleright, \unrhd$ are defined accordingly.

Given two $d$-indices $\boldsymbol{i}, \boldsymbol{j}$, we write $\boldsymbol{i}<\boldsymbol{j}$ if $i_{r}<j_{r}$ for every $r=1, \ldots, d$. The relations $\leq,>, \geq$ are defined accordingly.

We use bold letters for vectors and vector/matrix-valued functions. We indicate with $\mathbf{0}, \mathbf{1}, \mathbf{2}, \ldots$, the $d$-dimensional constant vectors $(0,0, \ldots, 0),(1,1, \ldots, 1)$, $(2,2, \ldots, 2), \ldots$, respectively. With the notation $\frac{\boldsymbol{i}}{\boldsymbol{n}}$ we mean the element-wise division of vectors, i.e., $\frac{\boldsymbol{i}}{\boldsymbol{n}}=\left(\frac{i_{1}}{n_{1}}, \ldots, \frac{i_{d}}{n_{d}}\right)$. We write $|\boldsymbol{i}|$ for the vector $\left(\left|i_{1}\right|, \ldots,\left|i_{d}\right|\right)$. Finally, given a $d$-index $\boldsymbol{n}$, we write $\boldsymbol{n} \rightarrow \infty$ meaning that $\min _{r=1, \ldots, d}\left\{n_{r}\right\} \rightarrow \infty$.

\subsection{Graphs}

We call a (finite) graph the quadruple $G=(V, E, w, \kappa)$, defined by

- a set of nodes $V=\left\{v_{1}, v_{2}, \ldots, v_{n}\right\}$;

- a weight function $w: V \times V \rightarrow \mathbb{R}$;

- a set of edges $E=\left\{\left(v_{i}, v_{j}\right) \mid v_{i}, v_{j} \in V, w\left(v_{i}, v_{j}\right) \neq 0\right\}$ between the nodes;

- a potential term $\kappa: V \rightarrow \mathbb{R}$.

The non-zero values $w\left(v_{i}, v_{j}\right)$ of the weight function $w$ are called weights associated with the edge $\left(v_{i}, v_{j}\right)$. Given an edge $e=\left(v_{i}, v_{j}\right) \in E$, the nodes $v_{i}, v_{j}$ are called end-nodes for the edge $e$. An edge $e \in E$ is said to be incident to a node $v_{i} \in V$ if there exists a node $v_{j} \neq v_{i}$ such that either $e=\left(v_{i}, v_{j}\right)$ or $e=\left(v_{j}, v_{i}\right)$. A walk of length $k$ in $G$ is a set of nodes $v_{i_{1}}, v_{i_{2}}, \ldots, v_{i_{k}}, v_{i_{k+1}}$ such that, for all $1 \leq r \leq k$, $\left(v_{i_{r}}, v_{i_{r+1}}\right) \in E$. A closed walk is a walk for which $v_{i_{1}}=v_{i_{k+1}}$. A path is a walk with no repeated nodes. A graph is connected if there is a walk connecting every pair of nodes.

A graph is said to be unweighted if $w\left(v_{i}, v_{j}\right) \in\{0,1\}$ for every $v_{i}, v_{j} \in V$. In that case the weight function $w$ is uniquely determined by edges belonging to $E$.

A graph is said to be undirected if the weight function $w$ is symmetric, i.e., for every couple of nodes $v_{i}, v_{j}$ we have $w\left(v_{i}, v_{j}\right)=w\left(v_{j}, v_{i}\right)$. In this case the edges $\left(v_{i}, v_{j}\right)$ and $\left(v_{j}, v_{i}\right)$ are considered equivalent and the edges are formed by unordered pairs of vertices. Two nodes $v_{i}, v_{j}$ of an undirected graph are said to be neighbors if $\left(v_{i}, v_{j}\right) \in E$ and we write $v_{i} \sim v_{j}$. On the contrary, if $\left(v_{i}, v_{j}\right) \notin E$, we write $v_{i} \nsim v_{j}$.

An undirected graph with unweighted edges and no self-loops (edges from a node to itself) is said to be simple. When dealing with simple graphs we use the simplified notation $G=(V, E)$. 
Every graph $G=(V, E, w, \kappa)$ with $\kappa \equiv 0$ can be represented as a matrix

$$
W=\left(w_{i, j}\right)_{i, j=1}^{n} \in \mathbb{R}^{n \times n}
$$

called the adjacency matrix of the graph. In particular, there is a bijection between the set of weight functions $w: V \times V \rightarrow \mathbb{R}$ and the set of a adjacency matrices $W \in \mathbb{R}^{n \times n}$.

The entries of the adjacency matrix $W$ are

$$
(W)_{i, j}=w\left(v_{i}, v_{j}\right), \quad \forall v_{i}, v_{j} \in V .
$$

In short, the adjacency matrix tells which nodes are connected and the 'weight' of the connection. If the graph does not admit self-loops, then the diagonal elements of the adjacency matrix are all equal to zero. In the particular case of an undirected graph, the associated adjacency matrix is symmetric, and thus its eigenvalues are real [4]. Moreover, the degree of a node $v_{i}$ of an undirected graph, denoted by $\operatorname{deg}\left(v_{i}\right)$, is defined as the sum of weights associated with edges incident to $v_{i}$, that is,

$$
\operatorname{deg}\left(v_{i}\right):=\sum_{v_{j} \sim v_{i}} w\left(v_{i}, v_{j}\right) .
$$

Given two graphs $G=(V, E, w, \kappa), G^{\prime}=\left(V^{\prime}, E^{\prime}, w^{\prime}, \kappa^{\prime}\right)$ with

$$
V=\left\{v_{1}, \ldots, v_{n}\right\}, \quad V^{\prime}=\left\{v_{1}^{\prime}, \ldots, v_{m}^{\prime}\right\},
$$

we say that $G$ is isomorphic to $G^{\prime}$, and we write $G \simeq G^{\prime}$, if

- $n=m$, i.e., $|V|=\left|V^{\prime}\right|$ where $|\cdot|$ is the cardinality of a set;

- there exists a permutation $P$ over the standard set $[n]:=\{1, \ldots, n\}$ such that

$$
w\left(v_{i}, v_{j}\right)=w^{\prime}\left(v_{P(i)}^{\prime}, v_{P(j)}^{\prime}\right), \quad \kappa\left(v_{i}\right)=\kappa^{\prime}\left(v_{P(i)}^{\prime}\right) .
$$

In short, two graphs are isomorphic if they contain the same number of vertices connected in the same way. Notice that an isomorphism between graphs is characterized by the permutation matrix $P$.

As an immediate consequence of the previous definition, it holds that $G \simeq G^{\prime}$ if and only if there exists a permutation matrix $P$ such that $W=P W^{\prime} P^{-1}=P W^{\prime} P^{T}$, where $W, W^{\prime}$ are the adjacency matrices of $G$ and $G^{\prime}$, respectively.

Definition 2.1 (Linking-graph operator). Given $\nu \in \mathbb{N}$, we call linking-graph operator for the reference node set $[\nu]:=\{1, \ldots, \nu\}$ any non-zero $\mathbb{R}^{\nu \times \nu}$ matrix, and we indicate it with $L$. Namely, a linking-graph operator is the adjacency matrix for a (possibly not undirected) graph $G=([\nu], E, l)$, with $l$ a weight function. When the entries of $L$ are just in $\{0,1\}$ we call it a simple linking-graph operator.

In Section 5, we use the linking-graph operator to connect a (infinite) sequence of graphs

$$
G_{1} \simeq G_{2} \simeq \ldots \simeq G_{n} \simeq \ldots,
$$

and to define the graph $G:=\bigcup_{n=1}^{\infty} G_{n}$ which has a uniform local structure. 
Sometimes it is useful to deal with proper sub-graphs. Given a graph $\bar{G}=$ $(\bar{V}, \bar{E}, \bar{w}, \bar{\kappa})$ and a subset $V \subset \bar{V}$, then

$$
\stackrel{\circ}{V}:=\left\{v_{i} \in V \mid v_{i} \nsim \bar{v}_{j} \forall \bar{v}_{j} \in \bar{V} \backslash V\right\}
$$

is called interior of $V$ and its elements are called interior nodes. Whereas, the set of nodes

$$
\partial V:=\left\{v_{i} \in V \mid v_{i} \sim \bar{v}_{j} \text { for some } \bar{v}_{j} \in \bar{V} \backslash V\right\}
$$

is called (internal) boundary of $V$ and its elements are called boundary nodes. We say that a graph $G=(V, E, w, \kappa)$ is a (proper) sub-graph of $\bar{G}$, and we write $G \subset \bar{G}$, if

- $V \subset \bar{V}$;

- $E=\left\{\left(v_{i}, v_{j}\right) \in \bar{E} \mid v_{i}, v_{j} \in V\right\} \subset \bar{E}$;

- $w=\bar{w}_{\mid E}$;

- $\kappa=\bar{\kappa}_{\mid V^{\circ}}$.

We call $\bar{G}$ the host graph. Observe that we do not request that $\kappa=\bar{\kappa}$ on $\partial V$.

Finally, the set of real functions on $V$ is denoted as $C(V)$. Trivially, $C(V)$ is isomorphic to $\mathbb{R}^{n}$. Of great importance for Section 7 is the operator $\Delta_{G}: C(V) \rightarrow$ $C(V)$ defined below.

Definition 2.2 (Graph-Laplacian). Given an undirected graph with no loops $G=$ $(V, E, w, \kappa)$, the graph-Laplacian is the symmetric matrix $\Delta_{G}: C(V) \rightarrow C(V)$ defined as

$$
\Delta_{G}:=D+K-W
$$

where $D$ is the degree matrix and $K$ is the potential term matrix, that is,

$$
D:=\operatorname{diag}\left\{\operatorname{deg}\left(v_{1}\right), \ldots, \operatorname{deg}\left(v_{n}\right)\right\}, \quad K:=\operatorname{diag}\left\{\kappa\left(v_{1}\right), \ldots, \kappa\left(v_{n}\right)\right\},
$$

and $W$ is the adjacency matrix of the graph $G$, that is,

$$
W=\left(\begin{array}{cccc}
0 & w\left(v_{1}, v_{2}\right) & \cdots & w\left(v_{1}, v_{n}\right) \\
w\left(v_{1}, v_{2}\right) & 0 & \ddots & \vdots \\
\vdots & \ddots & \ddots & w\left(v_{n-1}, v_{n}\right) \\
w\left(v_{1}, v_{n}\right) & \cdots & w\left(v_{n-1}, v_{n}\right) & 0
\end{array}\right)
$$

Namely,

$$
\Delta_{G}=\left(\begin{array}{cccc}
\operatorname{deg}\left(v_{1}\right)+\kappa\left(v_{1}\right) & -w\left(v_{1}, v_{2}\right) & \cdots & -w\left(v_{1}, v_{n}\right) \\
-w\left(v_{1}, v_{2}\right) & \operatorname{deg}\left(v_{2}\right)+\kappa\left(v_{2}\right) & \ddots & \vdots \\
\vdots & \ddots & \ddots & -w\left(v_{n-1}, v_{n}\right) \\
-w\left(v_{1}, v_{n}\right) & \cdots & -w\left(v_{n-1}, v_{n}\right) & \operatorname{deg}\left(v_{n}\right)+\kappa\left(v_{n}\right)
\end{array}\right) .
$$




\subsection{Toeplitz matrices, $d$-level Toeplitz matrices, and symbols}

Toeplitz matrices $T_{n}$ are characterized by the fact that all their diagonals parallel to the main diagonal have constant values: $\left(T_{n}\right)_{i, j}=\mathrm{t}_{i-j}$, where $i, j=1, \ldots, n$, for given coefficients $\mathrm{t}_{k}, k=1-n, \ldots, n-1$ :

$$
T_{n}=\left(\begin{array}{cccc}
\mathrm{t}_{0} & \mathrm{t}_{-1} & \cdots & \mathrm{t}_{1-n} \\
\mathrm{t}_{1} & \mathrm{t}_{0} & \ddots & \vdots \\
\vdots & \ddots & \ddots & \mathrm{t}_{-1} \\
\mathrm{t}_{n-1} & \cdots & \mathrm{t}_{1} & \mathrm{t}_{0}
\end{array}\right) .
$$

When every term $t_{k}$ is a matrix of fixed size $\nu$, i.e., $\mathrm{t}_{k} \in \mathbb{C}^{\nu \times \nu}$, the matrix $T_{n}$ is of block Toeplitz type. Owing to its intrinsic recursive nature, the definition of $d$ level (block) Toeplitz matrices is definitely more involved. More precisely, a $d$-level Toeplitz matrix is a Toeplitz matrix where each coefficient $t_{k}$ denotes a $(d-1)$-level Toeplitz matrix and so on in a recursive manner. In a more formal detailed way, using a standard multi-index notation introduced at the beginning of Section 2, a $d$-level Toeplitz matrix is of the form

$$
T_{\boldsymbol{n}}=\left(\mathrm{t}_{\boldsymbol{i}-\boldsymbol{j}}\right)_{\boldsymbol{i}, \boldsymbol{j = 1}}^{\boldsymbol{n}} \in \mathbb{C}^{\left(n_{1} \cdots n_{d}\right) \times\left(n_{1} \cdots n_{d}\right)},
$$

with the multi-index $\boldsymbol{n}$ such that $\mathbf{0}<\boldsymbol{n}=\left(n_{1}, \ldots, n_{d}\right)$ and $\mathrm{t}_{\boldsymbol{k}} \in \mathbb{C},-(\boldsymbol{n}-\mathbf{1}) \unlhd \boldsymbol{k} \unlhd$ $\boldsymbol{n}-\mathbf{1}$. If the basic elements $\mathrm{t}_{\boldsymbol{k}}$ denote blocks of a fixed size $\nu \geq 2$, i.e. $\mathrm{t}_{\boldsymbol{k}} \in \mathbb{C}^{\nu \times \nu}$, then $T_{\boldsymbol{n}, \nu}$ is a $d$-level block Toeplitz matrix,

$$
T_{\boldsymbol{n}, \nu}=\left(\mathrm{t}_{\boldsymbol{i}-\boldsymbol{j}}\right)_{\boldsymbol{i}, \boldsymbol{j}=\mathbf{1}}^{\boldsymbol{n}} \in \mathbb{C}^{\left(n_{1} \cdots n_{d} \nu\right) \times\left(n_{1} \cdots n_{d} \nu\right)}, \quad \mathrm{t}_{\boldsymbol{k}} \in \mathbb{C}^{\nu \times \nu} .
$$

For the sake of simplicity, we write down an explicit example with $d=2$ and $\nu=3$ :

$$
\begin{gathered}
T_{\boldsymbol{n}, 3}=\left(\begin{array}{cccc}
T_{0} & T_{-1} & \cdots & T_{1-n_{1}} \\
T_{1} & T_{0} & \ddots & \vdots \\
\vdots & \ddots & \ddots & T_{-1} \\
T_{n_{1}-1} & \cdots & T_{1} & T_{0}
\end{array}\right), \quad T_{k_{1}}=\left(\begin{array}{cccc}
\mathrm{t}_{k_{1}, 0} & \mathrm{t}_{k_{1},-1} & \cdots & \mathrm{t}_{k_{1}, 1-n_{2}} \\
\mathrm{t}_{k_{1}, 1} & \mathrm{t}_{k_{1}, 0} & \ddots & \vdots \\
\vdots & \ddots & \ddots & \mathrm{t}_{k_{1},-1} \\
\mathrm{t}_{k_{1}, n_{2}-1} & \cdots & \mathrm{t}_{k_{1}, 1} & \mathrm{t}_{k_{1}, 0}
\end{array}\right), \\
\mathrm{t}_{k_{1}, k_{2}} \in \mathbb{C}^{3 \times 3}, \quad k_{1} \in\left\{1-n_{1}, \ldots, n_{1}-1\right\}, \quad k_{2} \in\left\{1-n_{2}, \ldots, n_{2}-1\right\} .
\end{gathered}
$$

Observe that each block $T_{k_{1}}$ has a (block) Toeplitz structure. When $\nu=1$, then we just write $T_{\boldsymbol{n}, \nu}=T_{\boldsymbol{n}}$.

Here we are interested in asymptotic results and thus it is important to a have a meaningful way for defining sequences of Toeplitz matrices, enjoying global common properties. A classical and successful possibility is given by the use of a fixed function, called the generating function, and by taking its Fourier coefficients as entries of all the matrices in the sequence.

More specifically, given a function $\boldsymbol{f}:[-\pi, \pi]^{d} \rightarrow \mathbb{C}^{\nu \times \nu}$ belonging to $\mathrm{L}^{1}\left([-\pi, \pi]^{d}\right)$, we denote its Fourier coefficients by

$$
\hat{\boldsymbol{f}}_{\boldsymbol{k}}=\frac{1}{(2 \pi)^{d}} \int_{[-\pi, \pi]^{d}} \boldsymbol{f}(\boldsymbol{\theta}) \mathrm{e}^{-\mathrm{i} \boldsymbol{k} \cdot \boldsymbol{\theta}} d \boldsymbol{\theta} \in \mathbb{C}^{\nu \times \nu}, \quad \boldsymbol{k} \in \mathbb{Z}^{d}, \quad \boldsymbol{k} \cdot \boldsymbol{\theta}=\sum_{r=1}^{d} k_{r} \theta_{r}
$$


(the integrals are done component-wise), and we associate to $f$ the family of $d$-level block Toeplitz matrices

$$
T_{\boldsymbol{n}, \nu}(\boldsymbol{f}):=\left(\hat{\boldsymbol{f}}_{\boldsymbol{i}-\boldsymbol{j}}\right)_{\boldsymbol{i}, \boldsymbol{j}=\mathbf{1}}^{\boldsymbol{n}}, \quad \boldsymbol{n} \in \mathbb{N}^{d}
$$

We call $\left\{T_{\boldsymbol{n}, \nu}(\boldsymbol{f})\right\}_{\boldsymbol{n}}$ the family of multilevel block Toeplitz matrices associated with the function $\boldsymbol{f}$, which is called the generating function of $\left\{T_{\boldsymbol{n}, \nu}(\boldsymbol{f})\right\}_{\boldsymbol{n}}$. If $\boldsymbol{f}$ is Hermitian matrix-valued, i.e. $\boldsymbol{f}(\boldsymbol{\theta})$ is Hermitian for almost every $\boldsymbol{\theta}$, then it is plain to see that all the matrices $T_{\boldsymbol{n}, \nu}(\boldsymbol{f})$ are Hermitian, simply because the Hermitian character of the generating function and relations (1) imply that $\hat{\boldsymbol{f}}_{-\boldsymbol{k}}=\hat{\boldsymbol{f}}_{\boldsymbol{k}}^{*}$ for all $\boldsymbol{k} \in \mathbb{Z}^{d}$, where the ${ }^{*}$-symbol indicates the complex conjugate transpose. If, in addition, $\boldsymbol{f}(\boldsymbol{\theta})=\boldsymbol{f}(|\boldsymbol{\theta}|)$ for every $\boldsymbol{\theta}$, then all the matrices $T_{\boldsymbol{n}}(\boldsymbol{f})$ are real symmetric with real symmetric blocks $\hat{\boldsymbol{f}}_{\boldsymbol{k}}, \boldsymbol{k} \in \mathbb{Z}^{d}$.

\subsection{Spectral symbol}

We say that a matrix-valued function $\mathfrak{f}: D \rightarrow \mathbb{C}^{\nu \times \nu}, \nu \geq 1$, defined on a measurable set $D \subseteq \mathbb{R}^{m}, m \in \mathbb{N}$, is measurable (resp. continuous, in $\mathrm{L}^{p}(D)$ ) if its components $\mathfrak{f}_{i, j}: D \rightarrow \mathbb{C}, i, j=1, \ldots, \nu$, are measurable (resp. continuous, in $\mathrm{L}^{p}(D)$ ). Let $\mu_{m}$ be the Lebesgue measure on $\mathbb{R}^{m}$ and let $C_{c}(\mathbb{R})$ be the set of continuous functions with bounded support defined over $\mathbb{R}$. Setting $d_{\boldsymbol{n}}$ the dimension of a square matrix $X_{\boldsymbol{n}, \nu}$, for $F \in C_{c}(\mathbb{R})$ we define

$$
\Sigma_{\sigma}\left(F, X_{\boldsymbol{n}, \nu}\right):=\frac{1}{d_{\boldsymbol{n}}} \sum_{k=1}^{d_{\boldsymbol{n}}} F\left(\sigma_{k}\left(X_{\boldsymbol{n}, \nu}\right)\right), \quad \Sigma_{\lambda}\left(F, X_{\boldsymbol{n}, \nu}\right):=\frac{1}{d_{\boldsymbol{n}}} \sum_{k=1}^{d_{\boldsymbol{n}}} F\left(\lambda_{k}\left(X_{\boldsymbol{n}, \nu}\right)\right),
$$

where $\sigma_{k}\left(X_{n, \nu}\right)$ and $\lambda_{k}\left(X_{n, \nu}\right)$ are the singular values and the (real) eigenvalues of $X_{\boldsymbol{n}, \nu}$, respectively, sorted in non-decreasing order.

Hereafter, symbols $\left\{X_{\boldsymbol{n}, \nu}\right\}_{\boldsymbol{n}},\left\{Y_{\boldsymbol{n}, \nu}\right\}_{\boldsymbol{n}},\left\{Z_{\boldsymbol{n}, \nu}\right\}_{\boldsymbol{n}}$, with $\nu$ a fixed parameter independent of $\boldsymbol{n}$, indicate sequences of square matrices of increasing dimensions, i.e., such that $d_{\boldsymbol{n}} \rightarrow \infty$ as $\boldsymbol{n} \rightarrow \infty$.

We say that a sequence $\left\{X_{\boldsymbol{n}, \nu}\right\}_{\boldsymbol{n}}$ is zero distributed if

$$
\lim _{n \rightarrow \infty} \Sigma_{\sigma}\left(F, X_{n, \nu}\right)=F(0) \quad \forall F \in C_{c}(\mathbb{R}),
$$

and we indicate it by $\left\{X_{\boldsymbol{n}, \nu}\right\}_{\boldsymbol{n}} \sim_{\sigma} 0$.

Definition 2.3 (Spectral symbol). Let $\left\{X_{n, \nu}\right\}_{n}$ be a sequence of matrices and let $\mathfrak{f}: D \rightarrow \mathbb{C}^{\nu \times \nu}$ be a measurable Hermitian matrix-valued function defined on the measurable set $D \subset \mathbb{R}^{m}$, with $0<\mu_{m}(D)<\infty$.

We say that $\left\{X_{\boldsymbol{n}, \nu}\right\}_{\boldsymbol{n}}$ is distributed like $\mathfrak{f}$ in the sense of eigenvalues, in symbols $\left\{X_{n, \nu}\right\}_{n} \sim_{\lambda} \mathfrak{f}$, if

$$
\lim _{\boldsymbol{n} \rightarrow \infty} \Sigma_{\lambda}\left(F, X_{\boldsymbol{n}, \nu}\right)=\frac{1}{\mu_{m}(D)} \int_{D} \sum_{k=1}^{\nu} F\left(\lambda_{k}(\mathfrak{f}(\boldsymbol{y}))\right) d \mu_{m}(\boldsymbol{y}), \quad \forall F \in C_{c}(\mathbb{R}),
$$


where $\lambda_{1}(\mathfrak{f}(\boldsymbol{y})), \ldots, \lambda_{\nu}(\mathfrak{f}(\boldsymbol{y}))$ are the eigenvalues of $\mathfrak{f}(\boldsymbol{y})$. Let us notice that, in the case $\nu=1$, the identity (3) reduces to

$$
\lim _{\boldsymbol{n} \rightarrow \infty} \Sigma_{\lambda}\left(F, X_{\boldsymbol{n}}\right)=\frac{1}{\mu_{m}(D)} \int_{D} F(\mathfrak{f}(\boldsymbol{y})) d \mu_{m}(\boldsymbol{y}), \quad \forall F \in C_{c}(\mathbb{R}) .
$$

We call $\mathfrak{f}$ the (spectral) symbol of $\left\{X_{\boldsymbol{n}, \nu}\right\}_{\boldsymbol{n}}$.

The following result on Toeplitz matrix-sequences linking the definition of symbol function and generating function is due to P. Tilli.

Theorem 2.1 ([42]). Given a function $\boldsymbol{f}:[-\pi, \pi]^{d} \rightarrow \mathbb{C}^{\nu \times \nu}$ belonging to $\mathrm{L}^{1}\left([-\pi, \pi]^{d}\right)$, then

$$
\left\{T_{\boldsymbol{n}, \nu}(\boldsymbol{f})\right\}_{\boldsymbol{n}} \sim_{\lambda} \mathfrak{f} \equiv \boldsymbol{f}
$$

that is the generating function of $\left\{T_{\boldsymbol{n}, \nu}(\boldsymbol{f})\right\}_{\boldsymbol{n}}$ coincides with its symbol according to Definition 2.3.

Since in this paper we work only with undirected graphs (i.e., graphs whose associated adjacency matrix is symmetric), we deal with Hermitian-valued symbol functions $\mathfrak{f}$ such that $\lambda_{k}(\mathfrak{f}(\boldsymbol{y}))$ are real-valued for every $\boldsymbol{y} \in D \subset \mathbb{R}^{m}$, and for every $k=1, \ldots, \nu$. See for example Propositions 4.2, 4.4, and Theorem 5.2.

The knowledge of the symbol function $\mathfrak{f}$ can give valuable insights on the distribution of eigenvalues of a sequence of matrices. We refer to Section 3 where a collection of theoretical results is presented, and to Section 7 and [1] where numerical experiments are provided.

Unfortunately, a generic matrix-sequence $\left\{X_{\boldsymbol{n}, \nu}\right\}_{\boldsymbol{n}}$ does not always own a Toeplitzlike structure and therefore we cannot predict beforehand whether it is distributed like a spectral symbol $\mathfrak{f}$ or not. The Generalized Locally Toeplitz (GLT) theory provides practical tools to extend the class of matrix-sequences satisfying equation (3) for a given symbol $\mathfrak{f}$.

In light of the purposes of the present work we give the main properties of block GLT sequences instead of the original formal definition, which can be found in [26], along with the properties listed below, for two main reasons. First, the original definition reported in [26] is rather involved and it requires introducing several other definitions such as "block LT operators" and "block LT sequences". Moreover, from a practical point of view, the following properties define the same set of matrixsequences as the formal definition, with the advantage of being much easier to use for practical purposes. In other words, the axioms (GLT 1) - (GLT 5) listed below represent an equivalent characterization of the whole class of block GLT matrixsequences.

Before doing so, let us introduce the definition of approximating class of sequences.

Let $\left\{X_{\boldsymbol{n}, \nu}\right\}_{\boldsymbol{n}}$ be a sequence of matrices of increasing dimension $d_{\boldsymbol{n}}$ and let $\left\{\left\{Y_{\boldsymbol{n}, \nu, m}\right\}_{\boldsymbol{n}}\right\}_{m}$ be a sequence of matrix-sequences of the same dimension $d_{\boldsymbol{n}}$. We say that $\left\{\left\{Y_{\boldsymbol{n}, \nu, m}\right\}_{\boldsymbol{n}}\right\}_{m}$ is an approximating class of sequences (a.c.s.) for $\left\{X_{\boldsymbol{n}, \nu}\right\}_{\boldsymbol{n}}$, and we write

$$
\left\{Y_{\boldsymbol{n}, \nu, m}\right\}_{\boldsymbol{n}} \rightarrow\left\{X_{\boldsymbol{n}, \nu}\right\}_{\boldsymbol{n}} \text { a.c.s. }
$$


if the following condition is met: for every $m$ there exists $\boldsymbol{n}_{m}$ such that, for $\boldsymbol{n}>\boldsymbol{n}_{m}$,

$$
\begin{gathered}
X_{\boldsymbol{n}, \nu}=Y_{\boldsymbol{n}, \nu, m}+R_{\boldsymbol{n}, \nu, m}+N_{\boldsymbol{n}, \nu, m}, \quad \operatorname{rank}\left(R_{\boldsymbol{n}, \nu, m}\right) \leq c_{1}(m) d_{\boldsymbol{n}}, \\
\left\|N_{\boldsymbol{n}, \nu, m}\right\| \leq c_{2}(m),
\end{gathered}
$$

where $\boldsymbol{n}_{m}, c_{1}(m), c_{2}(m)$ depend only on $m$, and

$$
\lim _{m \rightarrow \infty} c_{1}(m)=\lim _{m \rightarrow \infty} c_{2}(m)=0 .
$$

In what follows we write $\left\{X_{\boldsymbol{n}, \nu}\right\}_{\boldsymbol{n}} \sim_{\mathrm{GLT}} \mathfrak{f}$ to indicate that $\left\{X_{\boldsymbol{n}, \nu}\right\}_{\boldsymbol{n}}$ is a block GLT sequence with symbol $\mathfrak{f}$, where $\mathfrak{f}: \Omega \times[-\pi, \pi]^{d} \subset \mathbb{R}^{2 d} \rightarrow \mathbb{C}$ is a measurable function, with $0<\mu_{d}(\Omega)<\infty$.

\section{Properties of block GLT sequences}

(GLT 1) If $\left\{X_{\boldsymbol{n}, \nu}\right\}_{\boldsymbol{n}} \sim_{\mathrm{GLT}} \mathfrak{f}$, then $\left\{X_{\boldsymbol{n}, \nu}\right\}_{\boldsymbol{n}} \sim_{\sigma} \mathfrak{f}$. Moreover, if each $X_{\boldsymbol{n}, \nu}$ is Hermitian, then $\left\{X_{\boldsymbol{n}, \nu}\right\}_{\boldsymbol{n}} \sim_{\lambda} \mathfrak{f}$.

(GLT 2) If $\left\{X_{\boldsymbol{n}, \nu}\right\}_{\boldsymbol{n}} \sim_{\mathrm{GLT}} \mathfrak{f}$ and $X_{\boldsymbol{n}, \nu}=Y_{\boldsymbol{n}, \nu}+Z_{\boldsymbol{n}, \nu}$, where

- every $Y_{\boldsymbol{n}, \nu}$ is Hermitian,

- $\left\|Y_{\boldsymbol{n}, \nu}\right\|,\left\|Z_{\boldsymbol{n}, \nu}\right\| \leq c$ for some constant $c$ independent of $d_{\boldsymbol{n}}$,

- $d_{\boldsymbol{n}}^{-1}\left\|Z_{\boldsymbol{n}, \nu}\right\|_{1} \rightarrow 0$,

then $\left\{X_{\boldsymbol{n}, \nu}\right\}_{\boldsymbol{n}} \sim_{\lambda} \mathfrak{f}$.

(GLT 3) We have:

- $\left\{T_{\boldsymbol{n}, \nu}(\boldsymbol{f})\right\}_{\boldsymbol{n}} \sim_{\mathrm{GLT}} \mathfrak{f} \equiv \boldsymbol{f}$ if $\boldsymbol{f}:[0,1]^{d} \rightarrow \mathbb{C}^{\nu \times \nu}$ is an integrable matrixvalued function;

- $\left\{\operatorname{diag}_{\boldsymbol{n}}(\boldsymbol{a})\right\}_{\boldsymbol{n}} \sim_{\mathrm{GLT}} \mathfrak{f} \equiv \boldsymbol{a}$ if $\boldsymbol{a}:[0,1]^{d} \rightarrow \mathbb{C}^{\nu \times \nu}$ is Riemann-integrable, where

$\operatorname{diag}_{\boldsymbol{n}}(\boldsymbol{a})=\left[\begin{array}{llll}\boldsymbol{a}\left(\frac{\mathbf{1}}{\boldsymbol{n}}\right) & & & \\ & \boldsymbol{a}\left(\frac{\mathbf{2}}{\boldsymbol{n}}\right) & & \\ & & \ddots & \\ & & & \boldsymbol{a}(\mathbf{1})\end{array}\right] \in \mathbb{C}^{\nu n_{1} \cdots n_{d} \times \nu n_{1} \cdots n_{d}} ;$

- $\left\{Z_{\boldsymbol{n}, \nu}\right\}_{\boldsymbol{n}} \sim_{\mathrm{GLT}} 0$ if and only if $\left\{X_{\boldsymbol{n}, \nu}\right\}_{\boldsymbol{n}} \sim_{\sigma} 0$.

(GLT 4) If $\left\{X_{\boldsymbol{n}, \nu}\right\}_{\boldsymbol{n}} \sim_{\mathrm{GLT}} \mathfrak{f}$ and $\left\{Y_{\boldsymbol{n}, \nu}\right\}_{\boldsymbol{n}} \sim_{\mathrm{GLT}} \mathfrak{g}$, then:

- $\left\{X_{\boldsymbol{n}, \nu}^{*}\right\}_{\boldsymbol{n}} \sim{ }_{\mathrm{GLT}} \mathfrak{f}^{*}$

- $\left\{\alpha X_{\boldsymbol{n}, \nu}+\beta Y_{\boldsymbol{n}, \nu}\right\}_{\boldsymbol{n}} \sim_{\mathrm{GLT}} \alpha \mathfrak{f}+\beta \mathfrak{g}$ for all $\alpha, \beta \in \mathbb{C}$;

- $\left\{X_{\boldsymbol{n}, \nu} Y_{\boldsymbol{n}, \nu}\right\}_{\boldsymbol{n}} \sim_{\mathrm{GLT}} \mathfrak{f} \mathfrak{g}$;

- $\left\{X_{\boldsymbol{n}, \nu}^{\dagger}\right\}_{\boldsymbol{n}} \sim_{\mathrm{GLT}} \mathfrak{f}^{-1}$ provided that $\mathfrak{f}$ is invertible a.e., where $X_{\boldsymbol{n}, \nu}^{\dagger}$ denotes the Moore-Penrose pseudoinverse of $X_{\boldsymbol{n}, \nu}$;

(GLT 5) $\left\{X_{\boldsymbol{n}, \nu}\right\}_{\boldsymbol{n}} \sim_{\mathrm{GLT}} \mathfrak{f}$ if and only if there exist a block GLT sequence

$\left\{\left\{Y_{\boldsymbol{n}, \nu, m}\right\}_{\boldsymbol{n}}\right\}_{m} \sim_{\mathrm{GLT}} \mathfrak{f}_{m}$ such that $\left\{\left\{Y_{\boldsymbol{n}, \nu, m}\right\}_{\boldsymbol{n}}\right\}_{m} \rightarrow\left\{X_{\boldsymbol{n}, \nu}\right\}_{\boldsymbol{n}}$ a.c.s. and $\mathfrak{f}_{m} \rightarrow \mathfrak{f}$ in measure.

\section{Weyl eigenvalue distribution}

Fix a square matrix-sequence $\left\{X_{\boldsymbol{n}, \nu}\right\}_{\boldsymbol{n}}$ of dimension $d_{\boldsymbol{n}}$, with symbol function $\mathfrak{f}$ : $D \subset \mathbb{R}^{m} \rightarrow \mathbb{C}^{\nu \times \nu}$ as in Definition 2.3. Observe that $\mathfrak{f}$ is not unique and in general not univariate. To avoid this, we introduce the notion of monotone rearrangement 
of the symbol, see Definition 3.1. In order to simplify the notation and since all the cases we investigate in this paper can be led back to this situation, we make the following assumptions:

\section{Assumptions}

(AS1) $\bar{D}$ is compact and of the form $\bar{\Omega} \times[-\pi, \pi]^{d}$ with $\bar{\Omega} \subseteq[0,1]^{d}$, and therefore $m=2 d$;

(AS2) $\mathfrak{f}(\boldsymbol{y})=\mathfrak{f}(\boldsymbol{x}, \boldsymbol{\theta})=p(\boldsymbol{x}) \boldsymbol{f}(\boldsymbol{\theta})$, with $(\boldsymbol{x}, \boldsymbol{\theta}) \in \Omega \times(-\pi, \pi)^{d}$ and $p: \Omega \rightarrow \mathbb{R}$, $f:(-\pi, \pi)^{d} \rightarrow \mathbb{C}^{\nu \times \nu}$

(AS3) $p: \Omega \rightarrow \mathbb{R}$ is piecewise continuous;

(AS4) every component $f_{i, j}:[-\pi, \pi]^{d} \rightarrow \mathbb{C}$ of $\boldsymbol{f}$ is continuous;

(AS5) $\boldsymbol{f}$ is a Hermitian matrix-valued function.

Because of (AS5) we are assuming that all the eigenvalues are real, then for notational convenience we order the eigenvalue functions $\lambda_{k}(p(\boldsymbol{x}) \boldsymbol{f}(\boldsymbol{\theta}))$ by magnitude, namely $\lambda_{1}(p(\boldsymbol{x}) \boldsymbol{f}(\boldsymbol{\theta})) \leq \ldots \leq \lambda_{\nu}(p(\boldsymbol{x}) \boldsymbol{f}(\boldsymbol{\theta}))$. This kind of ordering could affect the global regularity of the eigenvalue functions, but it does not affect the global regularity of the monotone rearrangement of the symbol, as we see in Theorem 3.2. Nevertheless, by well-known results (see [31]), items (AS3) and (AS4) imply that $\lambda_{k}(p(\boldsymbol{x}) \boldsymbol{f}(\boldsymbol{\theta}))$ is at least piecewise continuous for every $k=1, \ldots, \nu$. We have the following result.

Lemma 3.1. Suppose that $\left\{X_{\boldsymbol{n}, \nu}\right\}_{\boldsymbol{n}} \sim_{\lambda} \mathfrak{f}(\boldsymbol{x}, \boldsymbol{\theta})=p(\boldsymbol{x}) \boldsymbol{f}(\boldsymbol{\theta})$ as in Definition 2.3, where $\mathfrak{f}: D \rightarrow \mathbb{C}^{\nu \times \nu}$ is a Hermitian matrix-valued function satisfying assumptions (AS1)-(AS5). Then

$$
\left\{X_{\boldsymbol{n}, \nu}\right\}_{\boldsymbol{n}} \sim_{\lambda} \mathfrak{f}(\boldsymbol{x}, \boldsymbol{\theta})=p(\boldsymbol{x}) \sum_{k=1}^{\nu} \lambda_{k}\left(\boldsymbol{f}_{k}(\boldsymbol{\theta})\right), \quad(\boldsymbol{x}, \boldsymbol{\theta}) \in \hat{D}
$$

where $\mathfrak{f}: \hat{D} \rightarrow \mathbb{R}$ is a real-valued function and

$$
\begin{aligned}
\hat{D}= & \Omega \times\left(\bigcup_{k=1}^{\nu} I_{k}\right), \\
I_{k}= & \underbrace{\left[\frac{(2(k-1)-\nu) \pi}{\nu}, \frac{(2 k-\nu) \pi}{\nu}\right] \times \cdots \times\left[\frac{(2(k-1)-\nu) \pi}{\nu}, \frac{(2 k-\nu) \pi}{\nu}\right]}_{\text {d-times }}, \\
& \boldsymbol{f}_{k}: I_{k} \rightarrow \mathbb{C}^{\nu \times \nu}, \quad \boldsymbol{f}_{k}(\boldsymbol{\theta})= \begin{cases}\boldsymbol{f}(\nu \boldsymbol{\theta}-(2 k-1-\nu) \boldsymbol{\pi}) & \text { if } \boldsymbol{\theta} \in I_{k}, \\
\mathbf{0} & \text { otherwise. }\end{cases}
\end{aligned}
$$

Proof. By the monotone convergence theorem, since every $F \in C_{c}(\mathbb{R})$ is limit of a monotone sequence of step functions, then to prove (4) it is sufficient to prove the validity of Definition 2.3 for $F=\mathbb{1}_{E}$, with $E$ a measurable subset of $\operatorname{supp}(F)$. We have that

$$
\frac{1}{\mu_{m}(D)} \iint_{D} \sum_{k=1}^{\nu} \mathbb{1}_{E}\left(\lambda_{k}(p(\boldsymbol{x}) \boldsymbol{f}(\boldsymbol{\theta}))\right) d \mu_{m}=\sum_{k=1}^{\nu} \frac{1}{\mu_{m}(D)} \iint_{D} \mathbb{1}_{E_{k}}(\boldsymbol{x}, \boldsymbol{\theta}) d \mu_{m},
$$


where

$$
\begin{aligned}
E_{k} & =\left\{(\boldsymbol{x}, \boldsymbol{\theta}) \in D: \lambda_{k}(p(\boldsymbol{x}) \boldsymbol{f}(\boldsymbol{\theta})) \in E\right\} \\
& =\left\{(\boldsymbol{x}, \boldsymbol{\theta}) \in D: p(\boldsymbol{x}) \lambda_{k}(\boldsymbol{f}(\boldsymbol{\theta})) \in E\right\} .
\end{aligned}
$$

For every $k=1, \ldots, \nu$, let us make the change of variables

$$
\begin{aligned}
& (\boldsymbol{x}, \boldsymbol{\theta}) \mapsto\left(\boldsymbol{x}, \nu^{-1}[\boldsymbol{\theta}+(2 k-1-\nu) \boldsymbol{\pi}]\right), \\
& \left\{\begin{array}{l}
\hat{E}_{k}=\left\{(\boldsymbol{x}, \boldsymbol{\theta}) \in \hat{D}_{k}: p(\boldsymbol{x}) \lambda_{k}\left(\boldsymbol{f}_{k}(\boldsymbol{\theta})\right) \in E\right\}, \\
\hat{D}_{k}=\Omega \times I_{k},
\end{array}\right.
\end{aligned}
$$

from which it follows that

$$
\begin{aligned}
\sum_{k=1}^{\nu} \frac{1}{\mu_{m}(D)} \iint_{D} \mathbb{1}_{E_{k}}(\boldsymbol{x}, \boldsymbol{\theta}) d \mu_{m} & =\sum_{k=1}^{\nu} \frac{1}{\nu^{d} \mu_{m}\left(\hat{D}_{k}\right)} \iint_{\hat{D}_{k}} \mathbb{1}_{\hat{E}_{k}}(\boldsymbol{x}, \boldsymbol{\theta}) d \mu_{m} \\
& =\frac{1}{\mu_{m}(\hat{D})} \sum_{k=1}^{\nu} \iint_{\hat{D}_{k}} \mathbb{1}_{\hat{E}_{k}}(\boldsymbol{x}, \boldsymbol{\theta}) d \mu_{m} \\
& =\frac{1}{\mu_{m}(\hat{D})} \iint_{\hat{D}} \sum_{k=1}^{\nu} \mathbb{1}_{I_{k}}(\boldsymbol{\theta}) \mathbb{1}_{\hat{E}_{k}}(\boldsymbol{x}, \boldsymbol{\theta}) d \mu_{m} \\
& =\frac{1}{\mu_{m}(\hat{D})} \iint_{\hat{D}} \mathbb{1}_{E}\left(\sum_{k=1}^{\nu} p(\boldsymbol{x}) \lambda_{k}\left(\boldsymbol{f}_{k}(\boldsymbol{\theta})\right)\right) d \mu_{m} .
\end{aligned}
$$

Trivially, the maps $I_{k} \ni \boldsymbol{\theta} \mapsto \nu \boldsymbol{\theta}-(2 k-1-\nu) \boldsymbol{\pi}$ are diffeomorphism between $I_{k}$ and $[-\pi, \pi]^{d}$. Therefore, the image set of $\sum_{k=1}^{\nu} \lambda_{k}\left(\boldsymbol{f}_{k}(\boldsymbol{\theta})\right)$ over $I_{k}$ is exactly the image set of $\lambda_{k}(\boldsymbol{f}(\boldsymbol{\theta}))$ over $[-\pi, \pi]^{d}$.

The next definition of monotone rearrangement is crucial for the understanding of the asymptotic distribution of eigenvalues of $\left\{X_{\boldsymbol{n}, \nu}\right\}_{\boldsymbol{n}}$.

Definition 3.1. Let $\nu \geq 1$ and using the same notation as in Lemma 3.1, define

$$
R_{\mathfrak{f}}=\left\{p(\boldsymbol{x}) \sum_{k=1}^{\nu} \lambda_{k}\left(\boldsymbol{f}_{k}(\boldsymbol{\theta})\right):(\boldsymbol{x}, \boldsymbol{\theta}) \in \bar{\Omega} \times[-\pi, \pi]^{d}\right\} .
$$

Let $\mathfrak{f}^{\dagger}:[0,1] \rightarrow\left[\min R_{\mathfrak{f}}, \max R_{\mathfrak{f}}\right]$ be such that

$$
\mathfrak{f}^{\dagger}(x)=\inf \left\{t \in\left[\min R_{\mathfrak{f}}, \max R_{\mathfrak{f}}\right]: \phi_{\mathfrak{f}}(t) \geq x\right\}
$$

where $\phi_{\mathfrak{f}}: \mathbb{R} \rightarrow[0,1]$,

$$
\phi_{\mathfrak{f}}(t):=\frac{1}{\mu_{m}(\hat{D})} \mu_{m}\left(\left\{(\boldsymbol{x}, \boldsymbol{\theta}) \in \hat{D}: p(\boldsymbol{x}) \sum_{k=1}^{\nu} \lambda_{k}\left(\boldsymbol{f}_{k}(\boldsymbol{\theta})\right) \leq t\right\}\right) .
$$

$\mathfrak{f}^{\dagger}(x)$ is the monotone rearrangement of $\mathfrak{f}(\boldsymbol{x}, \boldsymbol{\theta})=p(\boldsymbol{x}) \sum_{k=1}^{\nu} \lambda_{k}\left(\boldsymbol{f}_{k}(\boldsymbol{\theta})\right)$. Because of Lemma 3.1, with abuse of notation we call $\mathfrak{f}^{\dagger}(x)$ the monotone rearrangement of $\mathfrak{f}(\boldsymbol{x}, \boldsymbol{\theta})=p(\boldsymbol{x}) \boldsymbol{f}(\boldsymbol{\theta})$ as well. In the special case that $\mathfrak{f}(\boldsymbol{x}, \boldsymbol{\theta})=\boldsymbol{f}(\boldsymbol{\theta})$, then $\mathfrak{f}^{\dagger} \equiv f^{\dagger}$. 
Clearly, $\mathfrak{f}^{\dagger}$ is well-defined, univariate, monotone strictly increasing and rightcontinuous. The common "analyst" notation for the monotone rearrangement of a function uses the star ${ }^{*}$-symbol. In this manuscript we prefer to use the dagger ${ }^{\dagger}$ symbol to avoid confusion with the conjugate transpose notation. Nevertheless, it is anyway appropriate since from a probabilistic point of view, $\mathfrak{f}^{\dagger}$ is the pseudo-inverse of the cumulative distribution function $\phi_{\mathfrak{f}}$.

Within our assumptions on $\mathfrak{f}$, it is easy to extend [14, Theorem 3.4] for this multi-variate matrix-valued case, and it holds that

(i) $\mathfrak{f}^{\dagger}(0)=\min R_{\mathfrak{f}}, \mathfrak{f}^{\dagger}(1)=\max R_{\mathfrak{f}}$;

(ii) $\lim _{\boldsymbol{n} \rightarrow \infty} \Sigma_{\lambda}\left(F, X_{\boldsymbol{n}, \nu}\right)=\int_{0}^{1} F\left(\mathfrak{f}^{\dagger}(x)\right) d \mu_{1}(x)$.

We have the following results, see [2, Section 3]. The statements and the techniques used in the proofs are almost the same, we mostly adjusted them to fit in the notation we are adopting here. In order to make the paper self-contained, we report here the sketches of the proofs.

Theorem 3.2. Let $\left\{X_{n, \nu}\right\}_{n}$ be a matrix-sequence such that

$$
\left\{X_{\boldsymbol{n}, \nu}\right\}_{\boldsymbol{n}} \sim_{\lambda} \mathfrak{f}(\boldsymbol{x}, \boldsymbol{\theta})=p(\boldsymbol{x}) \boldsymbol{f}(\boldsymbol{\theta}) .
$$

Suppose that

$$
\mu_{m}\left(\left\{(\boldsymbol{x}, \boldsymbol{\theta}) \in \bar{\Omega} \times[-\pi, \pi]^{d}: p(\boldsymbol{x}) \sum_{k=1}^{\nu} \lambda_{k}\left(\boldsymbol{f}_{k}(\boldsymbol{\theta})\right)=t\right\}\right)=0 \quad \forall t \in R_{\mathfrak{f}}
$$

(or, equivalently, that $\phi_{\mathfrak{f}}$ is continuous). Then

$$
\begin{gathered}
\left\{X_{\boldsymbol{n}, \nu}\right\}_{\boldsymbol{n}} \sim_{\lambda} \mathfrak{f}^{\dagger}(x), \quad x \in(0,1) ; \\
\lim _{\boldsymbol{n} \rightarrow \infty} \frac{\left|\left\{k=1, \ldots, d_{\boldsymbol{n}}: \lambda_{k}\left(X_{\boldsymbol{n}, \nu}\right) \leq t\right\}\right|}{d_{\boldsymbol{n}}}=\phi_{\mathfrak{f}}(t), \quad \forall t \in \mathbb{R} .
\end{gathered}
$$

Let $k=k(\boldsymbol{n})$ be such that $k(\boldsymbol{n}) / d_{\boldsymbol{n}} \rightarrow x_{0}^{-} \in(0,1)$ as $\boldsymbol{n} \rightarrow \infty$. Then

$$
\lim _{\boldsymbol{n} \rightarrow \infty} \lambda_{k(\boldsymbol{n})}\left(X_{\boldsymbol{n}, \nu}\right)=t_{0} \in\left(\min R_{\mathfrak{f}}, \max R_{\mathfrak{f}}\right), \quad \sup _{t \in R_{\mathfrak{f}}}\left\{t \leq t_{0}\right\}=\lim _{x \rightarrow x_{0}^{-}} \mathfrak{f}^{\dagger}(x) .
$$

In particular, if $\mathfrak{f}^{\dagger}$ is continuous in $x_{0}$, then

$$
\lim _{\boldsymbol{n} \rightarrow \infty} \lambda_{k(\boldsymbol{n})}\left(X_{\boldsymbol{n}, \nu}\right)=\lim _{\boldsymbol{n} \rightarrow \infty} \mathfrak{f}^{\dagger}\left(\frac{k(\boldsymbol{n})}{d_{\boldsymbol{n}}}\right)=\mathfrak{f}^{\dagger}\left(x_{0}\right) .
$$

Finally, if $\lambda_{k(\boldsymbol{n})}\left(X_{\boldsymbol{n}, \nu}\right) \geq \min \left(R_{\mathfrak{f}}\right)\left(\leq \max \left(R_{\mathfrak{f}}\right)\right)$ definitely, then equation (7) holds for $x_{0}=0\left(x_{0}=1\right)$ as well.

Proof. Because of Lemma 3.1, it holds that

$$
\left\{X_{\boldsymbol{n}, \nu}\right\}_{\boldsymbol{n}} \sim_{\lambda} \mathfrak{f}(\boldsymbol{x}, \boldsymbol{\theta})=p(\boldsymbol{x}) \sum_{k=1}^{\nu} \lambda_{k}\left(\boldsymbol{f}_{k}(\boldsymbol{\theta})\right) .
$$


By hypothesis, $\phi_{\mathfrak{f}}$ is continuous and then by standard results in Probability Theory it holds that $\phi_{\mathfrak{f}} \circ \mathfrak{f}:=X$ is uniformly distributed on $(0,1)$, i.e. $X \sim U(0,1)$, which implies that $\mathfrak{f}^{\dagger}(X)$ and $\mathfrak{f}$ have the same distribution. Therefore,

$\frac{1}{\mu_{m}(\hat{D})} \iint_{\hat{D}} F(\mathfrak{f}(\boldsymbol{x}, \boldsymbol{\theta})) d \mu_{m}(\boldsymbol{x}, \boldsymbol{\theta})=\mathbb{E}(F(\mathfrak{f}))=\mathbb{E}\left(F\left(\mathfrak{f}^{\dagger}(X)\right)\right)=\int_{0}^{1} F\left(\mathfrak{f}^{\dagger}(x)\right) d \mu_{1}(x)$

for every $F \in C_{c}(\mathbb{R})$. The above identity, combined with (4) and Definition 2.3, gives (6a). Define now

$$
\begin{aligned}
\phi_{\boldsymbol{n}}(t) & :=\frac{N\left(X_{\boldsymbol{n}, \nu}, t\right)}{d_{\boldsymbol{n}}}=\frac{\left|\left\{k=1, \ldots, d_{\boldsymbol{n}}: \lambda_{k}\left(X_{\boldsymbol{n}, \nu}\right) \leq t\right\}\right|}{d_{\boldsymbol{n}}}, \\
\mu_{\boldsymbol{n}}(\cdot) & :=\frac{1}{d_{\boldsymbol{n}}} \sum_{k=1}^{d_{\boldsymbol{n}}} \mathbb{1}_{\left\{\lambda_{k}\left(X^{(\boldsymbol{n})}\right)\right\}}(\cdot) .
\end{aligned}
$$

It holds that $\mu_{\boldsymbol{n}}$ is a sub-probability measure and that $\phi_{\boldsymbol{n}}$ is the distribution function of $\mu_{\boldsymbol{n}}$, that is $\mu_{\boldsymbol{n}}((-\infty, t])=\phi_{\boldsymbol{n}}(t)$ for every $t \in \mathbb{R}$. Combining now (4) and $[9$, Theorem 4.4.1], it is not difficult to prove that $\mu_{\boldsymbol{n}}$ converges vaguely (see $[9$, p. 85 and Theorem 4.3.1]) to $\mu_{\mathfrak{f}}$, the probability measure on $\mathbb{R}$ associated with $\phi_{\mathfrak{f}}$, i.e., such that $\mu_{\mathfrak{f}}(-\infty, t]=\phi_{\mathfrak{f}}(t)$ for every $t \in \mathbb{R}$. Then,

$$
\lim _{\boldsymbol{n} \rightarrow \infty} \frac{N\left(X_{\boldsymbol{n}, \nu}, t\right)}{d_{\boldsymbol{n}}}=\lim _{\boldsymbol{n} \rightarrow \infty} \phi_{\boldsymbol{n}}(t)=\lim _{\boldsymbol{n} \rightarrow \infty} \mu_{\boldsymbol{n}}(-\infty, t]=\mu_{\mathfrak{f}}(-\infty, t]=\phi_{\mathfrak{f}}(t),
$$

for every $t \in \mathbb{R}$, which is exactly $(6 \mathrm{~b})$.

Define $\lambda_{k(\boldsymbol{n})}:=\lambda_{k(\boldsymbol{n})}\left(X_{\boldsymbol{n}, \nu}\right)$. By equation $(6 \mathrm{~b})$ and since $\phi_{\mathfrak{f}}$ is continuous, by a well known theorem of Pólya it holds that $\phi_{\boldsymbol{n}} \rightarrow \phi_{\mathfrak{f}}$ uniformly. On the other hand, it holds that

$$
x_{0}=\lim _{\boldsymbol{n} \rightarrow \infty} \frac{k(\boldsymbol{n})}{d_{\boldsymbol{n}}}=\lim _{\boldsymbol{n} \rightarrow \infty} \frac{\left.N\left(X_{\boldsymbol{n}, \nu}, \lambda_{k(\boldsymbol{n})}\right)\right)}{d_{\boldsymbol{n}}}=\lim _{\boldsymbol{n} \rightarrow \infty} \phi_{\boldsymbol{n}}\left(\lambda_{k(\boldsymbol{n})}\right) .
$$

Therefore, for every $\epsilon>0$ there exist $N_{1}=N_{1}(\epsilon), N_{2}=N_{2}(\epsilon) \in \mathbb{N}$ such that

$$
\begin{gathered}
\sup _{t \in \mathbb{R}}\left|\phi_{\boldsymbol{n}}(t)-\phi_{\mathfrak{f}}(t)\right|<\epsilon \quad \forall \boldsymbol{n}=\left(n_{1}, \ldots, n_{d}\right) \text { such that } \min _{r=1, \ldots, d} n_{r}>N_{1}, \\
\left|\phi_{\boldsymbol{n}}\left(\lambda_{k(\boldsymbol{n})}\right)-x_{0}\right|<\epsilon \quad \forall \boldsymbol{n}=\left(n_{1}, \ldots, n_{d}\right) \text { such that } \min _{r=1, \ldots, d} n_{r}>N_{2} .
\end{gathered}
$$

It follows easily that

$$
\lim _{\boldsymbol{n} \rightarrow \infty} \phi_{\mathfrak{f}}\left(\lambda_{k(\boldsymbol{n})}\right)=\phi_{\mathfrak{f}}\left(\lim _{\boldsymbol{n} \rightarrow \infty} \lambda_{k(\boldsymbol{n})}\right)=x_{0} .
$$

Since $x_{0} \in(0,1)$, then by $(5 \mathrm{~b})$ it holds that

$$
\lim _{\boldsymbol{n} \rightarrow \infty} \lambda_{k(\boldsymbol{n})}=t_{0} \in\left(\min R_{\mathfrak{f}}, \max R_{\mathfrak{f}}\right) .
$$

Finally, by the above relation and by (5a), we can conclude that

$$
\lim _{x \rightarrow x_{0}^{-}} \mathfrak{f}^{\dagger}(x)=\lim _{\boldsymbol{n} \rightarrow \infty} \mathfrak{f}^{\dagger}\left(\frac{k(\boldsymbol{n})}{d_{\boldsymbol{n}}}\right)=\sup _{t \in R_{\mathfrak{f}}}\left\{t \leq t_{0}\right\}
$$


Let us observe now that $x_{0}$ is a jump discontinuity point for $\mathfrak{f}^{\dagger}$ if and only if there exist $t_{1}<t_{2} \in R_{\mathfrak{f}}$ such that $R_{\mathfrak{f}} \subseteq\left[\min R_{\mathfrak{f}}, t_{1}\right] \cup\left[t_{2}, \max R_{\mathfrak{f}}\right]$ and $\phi_{\mathfrak{f}}(t)=x_{0}$ if and only if $t \in\left[t_{1}, t_{2}\right]$. Therefore, if $\mathfrak{f}^{\dagger}$ is continuous in $x_{0}$, then $t_{0}=t_{1}=t_{2} \in R_{\mathfrak{f}}$ and we have (7).

Corollary 3.3. With the same hypothesis as in Theorem 3.2, it holds that

$$
\lim _{n \rightarrow \infty} \frac{\left|\left\{k=1, \ldots, d_{\boldsymbol{n}}: \lambda_{k}\left(X_{\boldsymbol{n}, \nu}\right) \notin R_{\mathfrak{f}}\right\}\right|}{d_{\boldsymbol{n}}}=0,
$$

that is, the number of possible outliers is o $\left(d_{\boldsymbol{n}}\right)$.

Proof. It is immediate from (6b). Let us observe that

$$
\begin{aligned}
0 \leq \lim _{\boldsymbol{n} \rightarrow \infty} \frac{\left|\left\{k: \lambda_{k}\left(X_{\boldsymbol{n}, \nu}\right)<\min R_{\mathfrak{f}}\right\}\right|}{d_{\boldsymbol{n}}} & \leq \lim _{n \rightarrow \infty} \frac{N\left(X_{\boldsymbol{n}, \nu}, \min R_{\mathfrak{f}}\right)}{d_{\boldsymbol{n}}} \\
& =\phi_{\mathfrak{f}}\left(\min R_{\mathfrak{f}}\right)=0 .
\end{aligned}
$$

Moreover, since

$$
\begin{aligned}
\frac{\left|\left\{k: \lambda_{k}\left(X_{\boldsymbol{n}, \nu}\right) \notin R_{\mathfrak{f}}\right\}\right|}{d_{\boldsymbol{n}}} & =\frac{\left|\left\{k: \lambda_{k}\left(X_{\boldsymbol{n}, \nu}\right) \in \mathbb{R}\right\}\right|}{d_{\boldsymbol{n}}}-\frac{\left|\left\{k: \lambda_{k}\left(X_{\boldsymbol{n}, \nu}\right) \in R_{\mathfrak{f}}\right\}\right|}{d_{\boldsymbol{n}}} \\
& =1-\frac{\left|\left\{k: \lambda_{k}\left(X_{\boldsymbol{n}, \nu}\right) \in R_{\mathfrak{f}}\right\}\right|}{d_{\boldsymbol{n}}} \\
& =1-\frac{N\left(X_{\boldsymbol{n}, \nu}, \max R_{\mathfrak{f}}\right)}{d_{\boldsymbol{n}}}+\frac{\left|\left\{k: \lambda_{k}\left(X_{\boldsymbol{n}, \nu}\right)<\min R_{\mathfrak{f}}\right\}\right|}{d_{\boldsymbol{n}}}
\end{aligned}
$$

then, passing to the limit, we get

$$
\lim _{\boldsymbol{n} \rightarrow \infty} \frac{\left|\left\{k: \lambda_{k}\left(X_{\boldsymbol{n}, \nu}\right) \notin R_{\mathfrak{f}}\right\}\right|}{d_{\boldsymbol{n}}}=1-\phi_{\mathfrak{f}}\left(\max R_{\mathfrak{f}}\right)=1-1=0 .
$$

Corollary 3.4. With the same hypothesis as in Theorem 3.2, assume moreover that $\mathfrak{f}^{\dagger}$ is absolutely continuous. Let $\tau:\left[\min R_{\mathfrak{f}}, \max R_{\mathfrak{f}}\right] \rightarrow \mathbb{R}$ be a differentiable real function and let $\{k(\boldsymbol{n})\}_{\boldsymbol{n}}$ be a sequence of integers such that

(i) $\frac{k(\boldsymbol{n})}{d_{\boldsymbol{n}}} \rightarrow x_{0} \in[0,1]$;

(ii) $\lambda_{k(\boldsymbol{n})+1}\left(X_{\boldsymbol{n}, \nu}\right)>\lambda_{k(\boldsymbol{n})}\left(X_{\boldsymbol{n}, \nu}\right) \in\left[\min R_{\mathfrak{f}}\right.$, $\left.\max R_{\mathfrak{f}}\right]$ definitely for $\boldsymbol{n} \rightarrow \infty$.

Then

$$
\lim _{\boldsymbol{n} \rightarrow \infty} d_{\boldsymbol{n}}\left[\tau\left(\lambda_{k(\boldsymbol{n})+1}\left(X_{\boldsymbol{n}, \nu}\right)\right)-\tau\left(\lambda_{k(\boldsymbol{n})}\left(X_{\boldsymbol{n}, \nu}\right)\right)\right]=\lim _{x \rightarrow x_{0}}\left(\tau\left(\mathfrak{f}^{\dagger}(x)\right)\right)^{\prime} \quad \text { a.e. }
$$

Proof. Since $\mathfrak{f}^{\dagger}$ is absolutely continuous then it is differentiable almost everywhere. Let $x_{0} \in[0,1]$ such that $\left(\mathfrak{f}^{\dagger}\right)_{\mid x=x_{0}}^{\prime}$ exists and such that $\mathfrak{f}^{\dagger}\left(x_{0}\right) \neq 0$. Then from (7),

$$
\lim _{\boldsymbol{n} \rightarrow \infty} \frac{\lambda_{k(\boldsymbol{n})}\left(X_{\boldsymbol{n}, \nu}\right)}{\mathfrak{f}^{\dagger}\left(\frac{k(\boldsymbol{n})}{d_{\boldsymbol{n}}}\right)}=1
$$


and we get:

$$
\begin{aligned}
\lim _{n \rightarrow \infty} \frac{\tau\left(\lambda_{k(\boldsymbol{n})+1}\left(X_{\boldsymbol{n}, \nu}\right)\right)-\tau\left(\lambda_{k(\boldsymbol{n})}\left(X_{\boldsymbol{n}, \nu}\right)\right)}{\frac{1}{d_{n}}} & =\lim _{n \rightarrow \infty} \frac{\tau\left(\mathfrak{f}^{\dagger}\left(\frac{k(\boldsymbol{n})}{d_{\boldsymbol{n}}}+\frac{1}{d_{\boldsymbol{n}}}\right)\right)-\tau\left(\mathfrak{f}^{\dagger}\left(\frac{k(\boldsymbol{n})}{d_{\boldsymbol{n}}}\right)\right)}{\frac{1}{d_{\boldsymbol{n}}}} \\
& =\lim _{\boldsymbol{n} \rightarrow \infty} \frac{\tau\left(\mathfrak{f}^{\dagger}\left(x_{0}+\frac{1}{d_{\boldsymbol{n}}}\right)\right)-\tau\left(\mathfrak{f}^{\dagger}\left(x_{0}\right)\right)}{\frac{1}{d_{\boldsymbol{n}}}} \\
& =\lim _{x \rightarrow x_{0}}\left(\tau\left(\mathfrak{f}^{\dagger}(x)\right)\right)^{\prime} .
\end{aligned}
$$

Since $\mu_{1}\left(\left\{x \in[0,1]: \nexists\left(\mathfrak{f}^{\dagger}\right)^{\prime}(x)\right.\right.$ or $\left.\left.\mathfrak{f}^{\dagger}(x)=0\right\}\right)=0$, we conclude.

Remark 3.1. It may often happen that $\mathfrak{f}^{\dagger}$ does not have an analytical expression or it is not feasible to calculate, therefore it is needed an approximation. The simplest and easiest way to obtain it is by mean of sorting in non-decreasing order a uniform sampling of the original symbol function $p(\boldsymbol{x}) f(\boldsymbol{\theta})$, in the case of real-valued symbol, or of sorting in non-decreasing order uniform samplings of $p(\boldsymbol{x}) \lambda_{k}(\boldsymbol{f}(\boldsymbol{\theta}))$ for $k=$ $1, \ldots, \nu$, in the case of a matrix-valued symbol. See [27, Section 3] and [22, Remark 2]. These approximations converge to $\mathfrak{f}^{\dagger}$ as the mesh-refinement goes to zero, see [40].

\section{Diamond Toeplitz graphs}

In this section we are going to present the main (local) graph-structure which is used to build more general graphs as union of sequences of sub-graphs, i.e., diamond Toeplitz graphs. The resulting graphs are then immersed in bounded regular domains of $\mathbb{R}^{d}$ in Section 5 . We proceed step by step, gradually increasing the complexity of the graph structure.

As a matter of reference, we have the following scheme of inclusions, with the related variable coefficient versions:

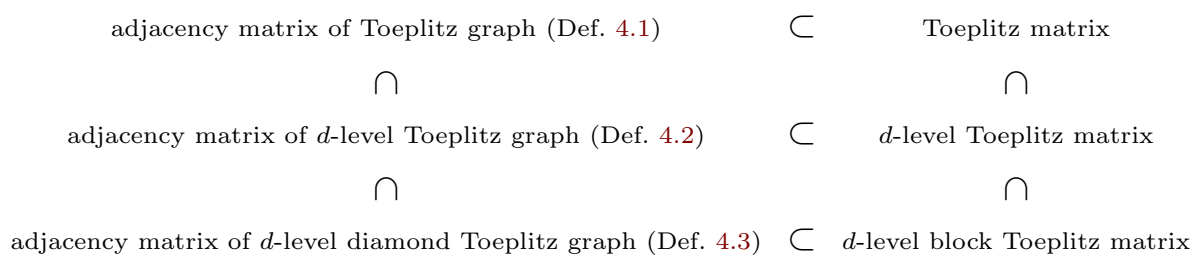

\subsection{Toeplitz graphs and $d$-level Toeplitz graphs}

We first focus on a particular type of graphs, namely Toeplitz graphs. These are graphs whose adjacency matrices are Toeplitz matrices.

Definition 4.1 (Toeplitz graph). Let $n, m, t_{1}, \ldots, t_{m}$ be positive integers such that $0<t_{1}<t_{2}<\ldots<t_{m}<n$, and fix $m$ nonzero real numbers $w_{t_{1}}, \ldots, w_{t_{m}}$. A Toeplitz 
graph, denoted by $T_{n}\left\langle\left(t_{1}, w_{t_{1}}\right), \ldots,\left(t_{m}, w_{t_{m}}\right)\right\rangle$, is an undirected graph defined by a node set $V_{n}=\left\{v_{1}, \ldots, v_{n}\right\}$ and a weight function $w$ such that

$$
w\left(v_{i}, v_{j}\right)= \begin{cases}w_{t_{k}} & \text { if }|i-j|=t_{k}, \\ 0 & \text { otherwise. }\end{cases}
$$

In the case of simple graphs, i.e., $w_{t_{k}}=1$ for every $k$, we indicate the Toeplitz graph just as $T_{n}\left\langle t_{1}, \ldots, t_{m}\right\rangle$. The number of edges in a Toeplitz graph is equal to $\sum_{k=1}^{m}\left(n-t_{k}\right)$. By construction, the adjacency matrix $W_{n}=\left(w_{|i-j|}\right)_{i, j=1}^{n}$ of a Toeplitz graph has a symmetric Toeplitz structure.

If we assume that $m, t_{1}, \ldots, t_{m}$, are fixed (independent of $n$ ) and we let the size $n$ grow, then the sequence of adjacency matrices $W_{n}$ can be related to a unique real integrable function $f$ (the symbol) defined on $[-\pi, \pi]$ and expanded periodically on $\mathbb{R}$. In this case, according to $(1)$, the entries $\left(W_{n}\right)_{i, j}=\hat{f}_{i-j}$ of the matrix $W_{n}$ are defined via the Fourier coefficients of $f$, where the $k$-th Fourier coefficient of $f$ is given by

$$
\hat{f}_{k}=\frac{1}{2 \pi} \int_{-\pi}^{\pi} f(\theta) e^{-\mathrm{i} k \theta} d \theta, \quad k \in \mathbb{Z} .
$$

We know that the Fourier coefficients $\hat{f}_{k}$ are all in $\left\{0, w_{t_{1}}, \ldots, w_{t_{m}}\right\}$ and that the matrix is symmetric. Note that obviously any such graph is uniquely defined by the first row of its adjacency matrix. On the other hand, we know that $w_{j-1}=$ $(W)_{1, j}=w\left(v_{1}, v_{j}\right)$ for $j=1, \ldots, n$, namely, $w_{j-1} \neq 0$ iff $j-1 \in\left\{t_{1}, \ldots, t_{m}\right\}$. From this condition we can infer that the symbol has a special polynomial structure and in fact it is equal to

$$
f(\theta)=\sum_{j=1-n}^{n-1} w_{|j|} e^{\mathrm{i} j \theta}=\sum_{k=1}^{m} 2 w_{t_{k}} \cos \left(t_{k} \theta\right) .
$$

In such a way, according to (8), our adjacency matrix $W_{n}$ is the matrix $T_{n}(f)$ (real and symmetric) having the following structure

$$
W_{n}=T_{n}(f)=\left(\begin{array}{cccc}
0 & w_{1} & \cdots & w_{n-1} \\
w_{1} & 0 & \ddots & \vdots \\
\vdots & \ddots & \ddots & w_{1} \\
w_{n-1} & \cdots & w_{1} & 0
\end{array}\right) \quad w_{j}= \begin{cases}w_{t_{k}} & \text { if } j=t_{k}, \\
0 & \text { otherwise }\end{cases}
$$

and, as expected, the symbol $f$ is real-valued and such that $f(\theta)=f(|\theta|)$ for every $\theta$. See Figure 1 for an example.

Along the same lines, we can define $d$-level Toeplitz graphs as a generalization of the Toeplitz graphs, but beforehand we need to define the set of directions associated with a $d$-index. Namely, given a $d$-index $\boldsymbol{t}_{k}=\left(\left(t_{k}\right)_{1}, \ldots,\left(t_{k}\right)_{d}\right)$ such that $\mathbf{0} \unlhd \boldsymbol{t}_{k}$ and $\boldsymbol{t} \neq \mathbf{0}$, define

$$
\begin{gathered}
I_{k}:=\left\{\boldsymbol{i} \in \mathbb{Z}^{d} \mid \boldsymbol{i}=\left( \pm\left(t_{k}\right)_{1}, \ldots, \pm\left(t_{k}\right)_{d}\right)\right\} \\
{\left[\boldsymbol{t}_{k}\right]:=I_{k} / \sim, \quad \text { where } \boldsymbol{i} \sim \boldsymbol{j} \text { iff } \boldsymbol{i}= \pm \boldsymbol{j} .}
\end{gathered}
$$




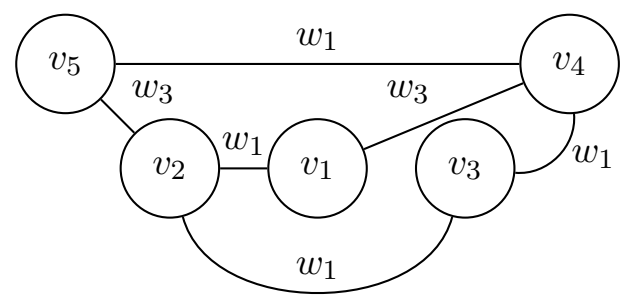

$$
W_{n}=\left(\begin{array}{ccccc}
0 & w_{1} & 0 & w_{3} & 0 \\
w_{1} & 0 & w_{1} & 0 & w_{3} \\
0 & w_{1} & 0 & w_{1} & 0 \\
w_{3} & 0 & w_{1} & 0 & w_{1} \\
0 & w_{3} & 0 & w_{1} & 0
\end{array}\right)
$$

Figure 1. Example of a 1-level Toeplitz graph $T_{n}\left\langle\left(1, w_{1}\right),\left(3, w_{3}\right)\right\rangle$, with $n=5$. The figure above is a visual representation of the graph while below it is explicated the associated adjacency matrix $W_{n}$ which presents the typical Toeplitz structure. In particular, $W_{n}$ has symbol function $f(\theta)=2 w_{1} \cos (\theta)+2 w_{3} \cos (3 \theta)$.

We call $\left[\boldsymbol{t}_{k}\right]$ the set of directions associated with $\boldsymbol{t}_{k}$.

Trivially, it holds that

$$
\left|I_{k}\right|=2^{\sum_{r=1}^{d} \mathbb{1}_{(0, \infty)}\left(\left|\left(t_{k}\right)_{r}\right|\right)}, \quad \text { where } \mathbb{1}_{(0, \infty)}(x)= \begin{cases}1 & \text { if } x \in(0, \infty) \\ 0 & \text { otherwise }\end{cases}
$$

and $\left|\left[\boldsymbol{t}_{k}\right]\right|=\left|I_{k}\right| / 2$. For $\alpha=1, \ldots,\left|\left[\boldsymbol{t}_{k}\right]\right|$, the elements $\left[\boldsymbol{t}_{k}\right]_{\alpha} \in\left[\boldsymbol{t}_{k}\right]$ are called directions and clearly $\left|\left[\boldsymbol{t}_{k}\right]_{\alpha}\right|=2$. We indicate with $\left[\boldsymbol{t}_{k}\right]_{\alpha}^{+}$the element in $\left[\boldsymbol{t}_{k}\right]_{\alpha}$ such that its first nonzero component is positive and with $\left[\boldsymbol{t}_{k}\right]_{\alpha}^{-}$the other one. Clearly, $-\left[\boldsymbol{t}_{k}\right]_{\alpha}^{+}=\left[\boldsymbol{t}_{k}\right]_{\alpha}^{-}$.

Definition 4.2 ( $d$-level Toeplitz graphs). Let $\boldsymbol{n}, \boldsymbol{t}_{1}, \ldots, \boldsymbol{t}_{m}$ be $d$-indices such that $\mathbf{0}<\boldsymbol{n}$, let

$$
0 \triangleleft \boldsymbol{t}_{1} \triangleleft \boldsymbol{t}_{2} \triangleleft \ldots \triangleleft \boldsymbol{t}_{m} \triangleleft \boldsymbol{n}-\mathbf{1},
$$

and fix $m$ nonzero real vectors $\boldsymbol{w}_{1}, \ldots, \boldsymbol{w}_{m}$, such that $\boldsymbol{w}_{k} \in \mathbb{R}^{c_{k}}$ with $c_{k}=\left|\left[\boldsymbol{t}_{k}\right]\right|$ for every $k=1, \ldots, m$, where $\left[\boldsymbol{t}_{k}\right]=\left\{\left[\boldsymbol{t}_{k}\right]_{1}, \ldots,\left[\boldsymbol{t}_{k}\right]_{c_{k}}\right\}$ is the set of directions associated with $\boldsymbol{t}_{k}$. We indicate the components of the vectors $\boldsymbol{w}_{k}$ using the following index notation,

$$
\boldsymbol{w}_{k}=\left(w_{\left[\boldsymbol{t}_{k}\right]_{1}}, w_{\left[\boldsymbol{t}_{k}\right]_{2}}, \ldots, w_{\left[\boldsymbol{t}_{k}\right]_{c_{k}}}\right) .
$$

A d-level Toeplitz graph, denoted by

$$
T_{\boldsymbol{n}}\left\langle\left\{\left[\boldsymbol{t}_{1}\right], \boldsymbol{w}_{1}\right\}, \ldots,\left\{\left[\boldsymbol{t}_{m}\right], \boldsymbol{w}_{m}\right\}\right\rangle,
$$

is an undirected graph defined by a node set $V_{\boldsymbol{n}}=\left\{v_{\boldsymbol{k}} \mid \mathbf{1} \unlhd \boldsymbol{k} \unlhd \boldsymbol{n}\right\}$ and a weight function $\omega$ such that

$$
w\left(v_{\boldsymbol{i}}, v_{\boldsymbol{j}}\right)= \begin{cases}w_{\left[\boldsymbol{t}_{k}\right]_{\alpha}} & \text { if }|\boldsymbol{i}-\boldsymbol{j}|=\boldsymbol{t}_{k} \text { and }(\boldsymbol{i}-\boldsymbol{j}) \in\left[\boldsymbol{t}_{k}\right]_{\alpha}=\left\{\left[\boldsymbol{t}_{k}\right]_{\alpha}^{+},\left[\boldsymbol{t}_{k}\right]_{\alpha}^{-}\right\} \\ & \text {for some } \alpha=1, \ldots, c_{k} \\ 0 & \text { otherwise }\end{cases}
$$


Let us observe that $w\left(v_{\boldsymbol{i}}, v_{\boldsymbol{j}}\right)=w\left(v_{\boldsymbol{j}}, v_{\boldsymbol{i}}\right)$, since $\boldsymbol{w}_{k}$ is defined over the classes of equivalence of $\left[\boldsymbol{t}_{k}\right]$. If there exist $m$ nonzero real numbers such that $\boldsymbol{w}_{k}=w_{k} \mathbf{1}$ for every $k=1, \ldots, m$, then the above relation translates into

$$
w\left(v_{\boldsymbol{i}}, v_{\boldsymbol{j}}\right)= \begin{cases}w_{k} & \text { if }|\boldsymbol{i}-\boldsymbol{j}|=\boldsymbol{t}_{k} \\ 0 & \text { otherwise }\end{cases}
$$

and we indicate the $d$-level Toeplitz graph as $T_{\boldsymbol{n}}\left\langle\left\{\boldsymbol{t}_{1}, w_{1}\right\}, \ldots,\left\{\boldsymbol{t}_{m}, w_{m}\right\}\right\rangle$. In the case of simple graph, i.e., $\boldsymbol{w}_{k}=\mathbf{1}$ for every $k$, we indicate the $d$-level Toeplitz graph just as $T_{n}\left\langle\boldsymbol{t}_{1}, \ldots, \boldsymbol{t}_{m}\right\rangle$. The number of nodes in a $d$-level Toeplitz graph is equal to $D(\boldsymbol{n})$ with $D(\boldsymbol{n})=\prod_{r=1}^{d} n_{r}$, while the number of edges is equal to $\sum_{r=1}^{m} D\left(\boldsymbol{n}-\boldsymbol{t}_{r}\right)$.

Lemma 4.1. A Toeplitz graph is a 1-level Toeplitz graph as in Definition 4.2.

Proof. We simply note that, for $d=1$, the quantities $\boldsymbol{n}, \boldsymbol{t}_{1}, \ldots, \boldsymbol{t}_{m}$ and the associated $\boldsymbol{w}_{1}, \ldots, \boldsymbol{w}_{m}$ are scalars, so that the resulting graph has $n$ points and weight function given by

$$
w\left(v_{i}, v_{j}\right)= \begin{cases}w_{t_{k}} & \text { if }|i-j|=t_{k} \\ 0 & \text { otherwise. }\end{cases}
$$

as in Definition 4.1, completing the proof.

If we assume that $m,\left\{\left[\boldsymbol{t}_{1}\right], \boldsymbol{w}_{1}\right\}, \ldots,\left\{\left[\boldsymbol{t}_{m}\right], \boldsymbol{w}_{m}\right\}$, are fixed (independent of $\boldsymbol{n}$ ) and we let the sizes $n_{j}$ grow, $j=1, \ldots, d$, then the sequence of adjacency matrices can be related to a unique real integrable function $f:[-\pi, \pi]^{d} \rightarrow \mathbb{R}$ (the symbol) and expanded periodically on $\mathbb{R}^{d}$. In this case, the entries $w_{\boldsymbol{i}, \boldsymbol{j}}=\hat{f}_{\boldsymbol{i}-\boldsymbol{j}}$ of the adjacency matrix are defined via the Fourier coefficients of $f$, where the $\boldsymbol{k}$-th Fourier coefficient of $f$ is defined according to the equations in (1). Following the same considerations which led to equation (8), we can summarize everything we said so far in the following proposition.

Proposition 4.2. Fix a d-level Toeplitz graph

$$
T_{\boldsymbol{n}}\left\langle\left\{\left[\boldsymbol{t}_{1}\right], \boldsymbol{w}_{1}\right\}, \ldots,\left\{\left[\boldsymbol{t}_{m}\right], \boldsymbol{w}_{m}\right\}\right\rangle,
$$

and assume that $m,\left\{\left[\boldsymbol{t}_{1}\right], \boldsymbol{w}_{1}\right\}, \ldots,\left\{\left[\boldsymbol{t}_{m}\right], \boldsymbol{w}_{m}\right\}$ are fixed and independent of $\boldsymbol{n}$. Then the adjacency matrix $W_{\boldsymbol{n}}$ of the graph is a symmetric matrix with a d-level Toeplitz structure (see Section 2.2),

$$
W_{\boldsymbol{n}}=\left(w_{\boldsymbol{i}-\boldsymbol{j}}\right)_{\boldsymbol{i}, \boldsymbol{j}=\mathbf{1}}^{\boldsymbol{n}}, \quad \text { where } w_{\boldsymbol{i}-\boldsymbol{j}}=w\left(v_{\boldsymbol{i}}, v_{\boldsymbol{j}}\right) \text {, as defined in (9). }
$$

In particular $W_{\boldsymbol{n}}=T_{\boldsymbol{n}}(f)$ with symbol function $f:[-\pi, \pi]^{d} \rightarrow \mathbb{R}$ given by

$$
f(\boldsymbol{\theta})=\sum_{k=1}^{m} \sum_{\alpha=1}^{c_{k}} 2 w_{\left[\boldsymbol{t}_{k}\right]_{\alpha}} \cos \left(\left[\boldsymbol{t}_{k}\right]_{\alpha}^{+} \cdot \boldsymbol{\theta}\right), \quad \text { with } c_{k}=\left|\left[\boldsymbol{t}_{k}\right]\right| \text { and } \boldsymbol{\theta}=\left(\theta_{1}, \ldots, \theta_{d}\right),
$$

that is,

$$
\left\{W_{\boldsymbol{n}}\right\}_{\boldsymbol{n}} \sim_{\lambda} \mathfrak{f} \equiv f
$$



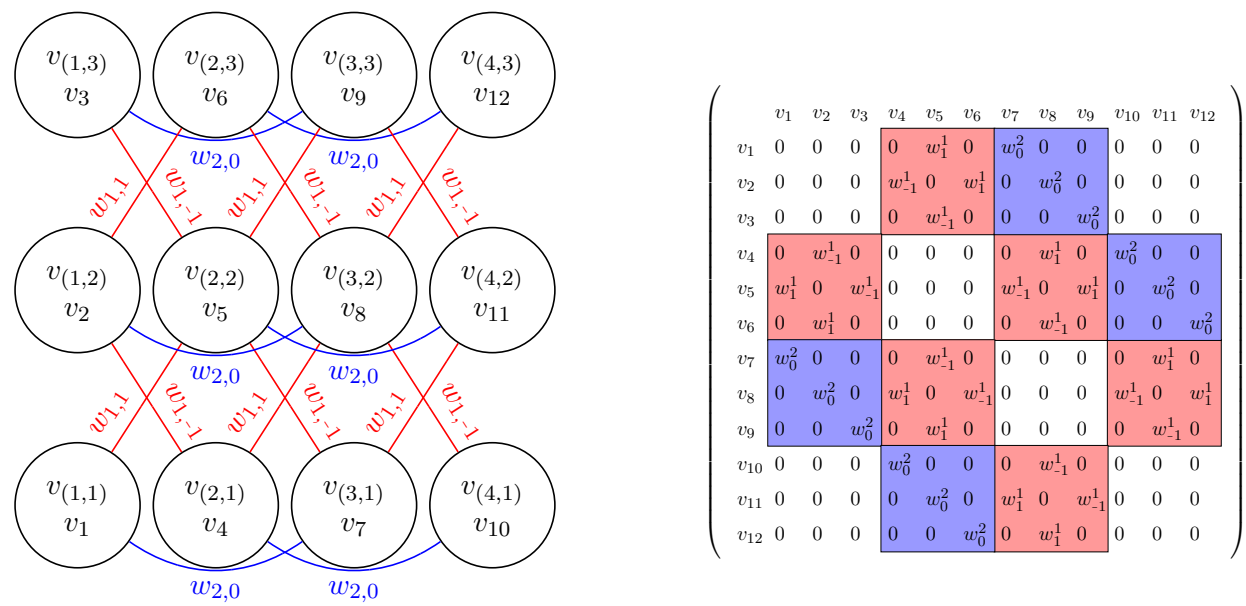

Figure 2. Example of a 2-level Toeplitz graph $T_{\boldsymbol{n}}\left\langle\left\{\left[\boldsymbol{t}_{1}\right], \boldsymbol{w}_{1}\right\},\left\{\left[\boldsymbol{t}_{2}\right], \boldsymbol{w}_{2}\right\}\right\rangle$ where $\boldsymbol{n}=(4,3),\left[\boldsymbol{t}_{1}\right]=[(1,1)]=\left\{[(1,1)]_{1},[(1,1)]_{2}\right\}, \boldsymbol{w}_{1}=$ $\left(w_{[(1,1)]_{1}}, w_{[(1,1)]_{2}}\right),\left[\boldsymbol{t}_{2}\right]=[(2,0)]=\left\{[(2,0)]_{1}\right\}$ and $\boldsymbol{w}_{2}=w_{[(2,0)]_{1}}$. In particular, $[(1,1)]_{1}=\{ \pm(1,-1)\},[(1,1)]_{2}=\{ \pm(1,1)\}$ and $[(2,0)]=\{ \pm(2,0)\}$. Combining the notation of (10) and (9), then we write $w_{[(1,1)]_{1}}=w_{1,-1}=w_{-1,1}, w_{[(1,1)]_{2}}=w_{1,1}=w_{-1,-1}$, $w_{[(2,0)]_{1}}=w_{2,0}=w_{-2,0}$. On the left there is a visual representation of the graph while on the right there is the associated adjacency matrix $W_{\boldsymbol{n}}$, where we used the standard lexicographic ordering to sort the nodes $\left\{v_{\boldsymbol{k}} \mid(1,1) \unlhd\left(k_{1}, k_{2}\right) \unlhd(4,3)\right\}$. Specifically, we write $v_{(1,1)}=v_{1}, v_{(1,2)}=v_{2}, \ldots, v_{(4,3)}=v_{12}$ and, for a better layout, we write $w_{1,-1}=w_{-1}^{1}, w_{1,1}=w_{1}^{1}, w_{2,0}=w_{0}^{2}$ for the adjacency matrix entries. $W_{\boldsymbol{n}}$ is a matrix which possesses a block Toeplitz with Toeplitz blocks (BTTB) structure and it has symbol function $f\left(\theta_{1}, \theta_{2}\right)=2 w_{2,0} \cos \left(2 \theta_{1}\right)+2 w_{1,1} \cos \left(\theta_{1}+\theta_{2}\right)+2 w_{1,-1} \cos \left(\theta_{1}-\theta_{2}\right)$ : notice that the coefficient of the variable $\theta_{1}$ refers to the diagonal blocks while the coefficient of $\theta_{2}$ refers to the diagonals inside the block. Finally, observe that $W_{\boldsymbol{n}}$ is not connected, since the graph can be decomposed into two disjoint subgraphs $G_{1}$ and $G_{2}$ having $\left\{v_{1}, v_{3}, v_{5}, v_{7}, v_{9}, v_{11}\right\}$ and $\left\{v_{2}, v_{4}, v_{6}, v_{8}, v_{10}, v_{12}\right\}$ as vertex sets, respectively.

Proof. The fact that

$$
W_{\boldsymbol{n}}=\left(w_{\boldsymbol{i}-\boldsymbol{j}}\right)_{\boldsymbol{i}, \boldsymbol{j}=\mathbf{1}}^{\boldsymbol{n}}
$$

is clear by Definition 4.2, while, by direct computation of the Fourier coefficients of $f$ and owing to the fact that $\cos \left(\left[\boldsymbol{t}_{k}\right]_{\alpha}^{+} \cdot \boldsymbol{\theta}\right)=\cos \left(\left[\boldsymbol{t}_{k}\right]_{\alpha}^{-} \cdot \boldsymbol{\theta}\right)$, we see that $\hat{f}_{\boldsymbol{i}-\boldsymbol{j}}=w_{\boldsymbol{i}-\boldsymbol{j}}=$ $w_{\boldsymbol{j}-\boldsymbol{i}}=\hat{f}_{\boldsymbol{j}-\boldsymbol{i}}$, so that $W_{\boldsymbol{n}}=T_{\boldsymbol{n}}(f)$.

See Figure 2 for an explicit example of a 2-level Toeplitz graph. 


\subsection{Graphs with uniform local structure: introducing the "diamond"}

The idea here is that each node in Definition 4.2 is replaced by a subgraph of fixed dimension $\nu$. For instance, fix a reference simple graph

$$
G=([\nu], E)
$$

with adjacency matrix $W$ and where $[\nu]:=\{1, \ldots, \nu\}$ is the standard set of cardinality $\nu \in \mathbb{N}$. Consider $0<n \in \mathbb{N}$ copies of such a graph, i.e., $G(k)=(V(k), E(k))$ such that $G(k) \simeq G$ for every $k=1, \ldots, n$. Indicating the distinct elements of each $V(k), k=1, \ldots, n$, with the notation $v_{(k, r)}$, for $r=1, \ldots, \nu$, we can define a new node set $V_{n}$ as the disjoint union of the sets $V(k)$, i.e.,

$$
V_{n}:=\bigsqcup_{k=1}^{n} V(k)=\left\{v_{(k, r)}:(1,1) \unlhd(k, r) \unlhd(n, \nu)\right\} .
$$

Fix now $m$ integers $0<t_{1}<\ldots<t_{m}$ with $1 \leq m \leq n-1$, and moreover fix $L_{t_{1}}, \ldots, L_{t_{m}}$ simple linking-graph operators for the reference node set $[\nu]:=$ $\{1, \ldots, \nu\}$, as in Definition 2.1, along with their uniquely determined edge sets $E_{t_{1}}, \ldots, E_{t_{m}} \subseteq[\nu] \times[\nu]$. Let us define the edge set $E_{n} \subseteq V_{n} \times V_{n},\left(v_{(i, r)}, v_{(j, s)}\right) \in E_{n}$ if and only if

$$
\begin{cases}i=j & \text { and }(r, s) \in E, \text { or } \\ i-j=t_{k} \text { for some } k=1, \ldots, m & \text { and }(r, s) \in E_{t_{k}, \text { or }} \\ i-j=-t_{k} \text { for some } k=1, \ldots, m & \text { and }(s, r) \in E_{t_{k}} .\end{cases}
$$

Namely, $E_{n}$ is the disjoint union of all the edge sets $E(k)$ plus all the edges which possibly connect nodes in a graph $G(i)$ with nodes in a graph $G(j)$ : two graphs $G(i), G(j)$ are connected if and only if $|i-j| \in\left\{t_{1}, \ldots, t_{m}\right\}$ and in that case the connection between the nodes of the two graphs is determined by the linking-graph operator $L_{t_{k}}$ (and by its transpose $L_{t_{k}}^{*}$ ). We can define then a kind of symmetric 'weight-graph function'

$$
\boldsymbol{w}:\{V(k) \mid k=1, \ldots, n\} \times\{V(k) \mid k=1, \ldots, n\} \rightarrow \mathbb{R}^{\nu \times \nu}
$$

such that

$$
\boldsymbol{w}[V(i), V(j)]:= \begin{cases}W & \text { if } i=j \\ L_{t_{k}} & \text { if } i-j \in\left\{t_{1}, \ldots, t_{m}\right\} \\ L_{t_{k}}^{*} & \text { if } i-j \in\left\{-t_{1}, \ldots,-t_{m}\right\} \\ 0 & \text { otherwise. }\end{cases}
$$

It is not difficult then to prove that the adjacency matrix $W_{n, \nu}^{G}$ of the graph $\left(V_{n}, E_{n}\right)$ is of the form

$$
W_{n, \nu}^{G}=\left(\begin{array}{cccc}
\boldsymbol{w}_{0} & \boldsymbol{w}_{1}^{*} & \cdots & \boldsymbol{w}_{n-1}^{*} \\
\boldsymbol{w}_{1} & \boldsymbol{w}_{0} & \ddots & \vdots \\
\vdots & \ddots & \ddots & \boldsymbol{w}_{1}^{*} \\
\boldsymbol{w}_{n-1} & \cdots & \boldsymbol{w}_{1} & \boldsymbol{w}_{0}
\end{array}\right), \quad \boldsymbol{w}_{j}= \begin{cases}W \in \mathbb{R}^{\nu \times \nu} & \text { if } j=0 \\
L_{t_{k}} \in \mathbb{R}^{\nu \times \nu} & \text { if } j=t_{k}, \\
\mathbf{0} & \text { otherwise. }\end{cases}
$$


Trivially, $W_{n, \nu}^{G}$ is a symmetric matrix with a block-Toeplitz structure and symbol function $\boldsymbol{f}$ given by

$$
\boldsymbol{f}(\theta)=W+\sum_{k=1}^{m}\left(L_{t_{k}}+L_{t_{k}}^{*}\right) \cos \left(t_{k} \theta\right)+\sum_{k=1}^{m}\left(L_{t_{k}}-L_{t_{k}}^{*}\right) \mathrm{i} \sin \left(t_{k} \theta\right) .
$$

Let us observe that $\boldsymbol{f}(\theta)$ is a Hermitian matrix in $\mathbb{C}^{\nu \times \nu}$ for every $\theta \in[-\pi, \pi]$, and therefore $\lambda_{j}(\boldsymbol{f}(\theta))$ are real for every $j=1, \ldots, \nu$, as we requested at the end of Subsection 2.3. We call

$$
T_{n}^{G}\left\langle\left(t_{1}, L_{t_{1}}\right), \ldots,\left(t_{m}, L_{t_{m}}\right)\right\rangle:=\left(V_{n}, E_{n}\right)
$$

a (simple) diamond Toeplitz graph associated with the graph $G$. A copy $G(k)$ of the graph $G$ is called $k$-th diamond.

See Figure 3 for an example. We can now generalize everything we said so far.

Definition 4.3 ( $d$-level diamond Toeplitz graph). Let $d, m, \nu$ be fixed integers and let $G \simeq([\nu], E, w)$ be a fixed undirected graph which we call mold graph.

Let $\boldsymbol{n}, \boldsymbol{t}_{1}, \ldots, \boldsymbol{t}_{m}$ be $d$-indices such that $\mathbf{0}<\boldsymbol{n}$, and $0 \triangleleft \boldsymbol{t}_{1} \triangleleft \boldsymbol{t}_{2} \triangleleft \ldots \triangleleft \boldsymbol{t}_{m} \triangleleft \boldsymbol{n}-\mathbf{1}$. For $k=1, \ldots, m$, let $\boldsymbol{L}_{k}$ be a collection of linking-graph operators of the standard set $[\nu]:=\{1, \ldots, \nu\}$ such that $\left|\boldsymbol{L}_{k}\right|=c_{k}$, with $c_{k}=\left|\left[\boldsymbol{t}_{k}\right]\right|$ for every $k=1, \ldots, m$, where $\left[\boldsymbol{t}_{k}\right]=\left\{\left[\boldsymbol{t}_{k}\right]_{1}, \ldots,\left[\boldsymbol{t}_{k}\right]_{c_{k}}\right\}$ is the set of directions associated with $\boldsymbol{t}_{k}$. We then indicate the elements of the set $\boldsymbol{L}_{k}$ by the following index notation,

$$
\begin{aligned}
& \boldsymbol{L}_{k}=\left\{L_{\left[\boldsymbol{t}_{k}\right]_{1}}, L_{\left[\boldsymbol{t}_{k}\right]_{2}}, \ldots, L_{\left[\boldsymbol{t}_{k}\right]_{c_{k}}}\right\}, \\
& \mathbb{R}^{\nu \times \nu} \ni L_{\left[\boldsymbol{t}_{k}\right]_{\alpha}}=\left(l_{\left[\boldsymbol{t}_{k}\right]_{\alpha}}(r, s)\right)_{r, s=1}^{\nu} \text { for } \alpha=1, \ldots, c_{k} .
\end{aligned}
$$

Finally, consider $\boldsymbol{n}$ copies $G(\boldsymbol{k}) \simeq G$ of the mold graph, which we call diamonds.

A d-level diamond Toeplitz graph, denoted by

$$
T_{\boldsymbol{n}, \nu}^{G}\left\langle\left\{\boldsymbol{t}_{1}, \boldsymbol{L}_{1}\right\}, \ldots,\left\{\boldsymbol{t}_{m}, \boldsymbol{L}_{m}\right\}\right\rangle,
$$

is an undirected graph with

$$
V_{\boldsymbol{n}}=\left\{v_{(\boldsymbol{k}, r)} \mid(\mathbf{1}, \mathbf{1}) \unlhd(\boldsymbol{k}, \mathbf{r}) \unlhd(\boldsymbol{n}, \nu)\right\}
$$

and characterized by the weight function $w_{\boldsymbol{n}}: V_{\boldsymbol{n}} \times V_{\boldsymbol{n}} \rightarrow \mathbb{R}$ such that

$$
w_{\boldsymbol{n}}\left(v_{(\boldsymbol{i}, r)}, v_{(\boldsymbol{j}, s)}\right):= \begin{cases}w(r, s) & \text { if } \boldsymbol{i}=\boldsymbol{j}, \\ l_{\left[\boldsymbol{t}_{k}\right]_{\alpha}}(r, s) & \text { if }|\boldsymbol{i}-\boldsymbol{j}|=\boldsymbol{t}_{k} \text { and }(\boldsymbol{i}-\boldsymbol{j})=\left[\boldsymbol{t}_{k}\right]_{\alpha}^{+}, \\ l_{\left[\boldsymbol{t}_{k}\right]_{\alpha}}(s, r) & \text { if }|\boldsymbol{i}-\boldsymbol{j}|=\boldsymbol{t}_{k} \text { and }(\boldsymbol{i}-\boldsymbol{j})=\left[\boldsymbol{t}_{k}\right]_{\alpha}^{-}, \\ 0 & \text { otherwise. }\end{cases}
$$

The number of nodes in a $d$-level diamond Toeplitz graph is equal to $\nu D(\boldsymbol{n})$ with $D(\boldsymbol{n})=\prod_{r=1}^{d} n_{r}$, while the number of edges is equal to $\nu \sum_{r=1}^{m} D\left(\boldsymbol{n}-\boldsymbol{t}_{r}\right)$.

Corollary 4.3. A d-level Toeplitz graph is a special case of a d-level diamond Toeplitz graph.

Proof. We simply need to notice that, for $\nu=1$, i.e., in the case of a diamond with only one element, the two Definitions 4.2 and 4.3 coincide with $\boldsymbol{L}_{k}=\boldsymbol{w}_{k}$. 
Proposition 4.4. Fix a d-level diamond Toeplitz graph

$$
T_{\boldsymbol{n}, \nu}^{G}\left\langle\left\{\boldsymbol{t}_{1}, \boldsymbol{L}_{1}\right\}, \ldots,\left\{\boldsymbol{t}_{m}, \boldsymbol{L}_{m}\right\}\right\rangle
$$

with $G \simeq([\nu], E, w)$ and $W$ the adjacency matrix of $G$.

Let $d, m, \nu,\left\{\boldsymbol{t}_{k}, \boldsymbol{L}_{k}\right\}, G$ be fixed and independent of $\boldsymbol{n}$. Then the adjacency matrix $W_{\boldsymbol{n}, \nu}^{G}$ of $T_{\boldsymbol{n}, \nu}^{G}\left\langle\left\{\boldsymbol{t}_{1}, \boldsymbol{L}_{1}\right\}, \ldots,\left\{\boldsymbol{t}_{m}, \boldsymbol{L}_{m}\right\}\right\rangle$ is a symmetric matrix with a d-level block Toeplitz structure (see Section 2.2 and equation (2)),

$$
W_{\boldsymbol{n}, \nu}^{G}=\left[\boldsymbol{w}_{\boldsymbol{i}-\boldsymbol{j}}\right]_{\boldsymbol{i}, \boldsymbol{j}=\mathbf{1}}^{\boldsymbol{n}}
$$

where

$$
\mathbb{R}^{\nu \times \nu} \ni \boldsymbol{w}_{\boldsymbol{i}-\boldsymbol{j}}= \begin{cases}W & \text { if } \boldsymbol{i}=\boldsymbol{j}, \\ L_{\left[\boldsymbol{t}_{k}\right]_{\alpha}} & \text { if }|\boldsymbol{i}-\boldsymbol{j}|=\boldsymbol{t}_{k} \text { and }(\boldsymbol{i}-\boldsymbol{j})=\left[\boldsymbol{t}_{k}\right]_{\alpha}^{+}, \\ L_{\left[\boldsymbol{t}_{k}\right]_{\alpha}}^{*} & \text { if }|\boldsymbol{i}-\boldsymbol{j}|=\boldsymbol{t}_{k} \text { and }(\boldsymbol{i}-\boldsymbol{j})=\left[\boldsymbol{t}_{k}\right]_{\alpha}^{-}, \\ \mathbf{0} & \text { otherwise. }\end{cases}
$$

In particular $W_{\boldsymbol{n}, \nu}^{G}=T_{\boldsymbol{n}, \nu}(\boldsymbol{f})$ with symbol function $\boldsymbol{f}:[-\pi, \pi]^{d} \rightarrow \mathbb{C}^{\nu \times \nu}$ given by

$$
\begin{aligned}
\boldsymbol{f}(\boldsymbol{\theta})= & W+\sum_{k=1}^{m} \sum_{\alpha=1}^{c_{k}}\left[\left(L_{\left[\boldsymbol{t}_{k}\right]_{\alpha}}+L_{\left[\boldsymbol{t}_{k}\right]_{\alpha}}^{*}\right) \cos \left(\boldsymbol{t}_{k} \cdot \boldsymbol{\theta}\right)\right. \\
& \left.+\left(L_{\left[\boldsymbol{t}_{k}\right]_{\alpha}}-L_{\left[\boldsymbol{t}_{k}\right]_{\alpha}}^{*}\right) i \sin \left(\boldsymbol{t}_{k} \cdot \boldsymbol{\theta}\right)\right], \quad \boldsymbol{\theta}=\left(\theta_{1}, \ldots, \theta_{d}\right)
\end{aligned}
$$

that is,

$$
\left\{W_{\boldsymbol{n}, \nu}^{G}\right\}_{\boldsymbol{n}} \sim_{\lambda} \mathfrak{f} \equiv \boldsymbol{f}
$$

The symbol function $\boldsymbol{f}$ is Hermitian matrix-valued for every $\boldsymbol{\theta} \in[-\pi, \pi]^{d}$.

Proof. We note that $W_{\boldsymbol{n}}^{G}=\left[\boldsymbol{w}_{\boldsymbol{i}-\boldsymbol{j}}\right]_{\boldsymbol{i}, \boldsymbol{j = 1}}^{\boldsymbol{n}}$ is immediate by Definition 4.3 and that the symbol $\boldsymbol{f}$ is a Hermitian matrix for every $\boldsymbol{\theta}$, so that it has real eigenvalues. Moreover we see that, as in Proposition $4.2, \hat{\boldsymbol{f}}_{\boldsymbol{i}-\boldsymbol{j}}=\boldsymbol{w}_{\boldsymbol{i}-\boldsymbol{j}}$. Now Theorem 2.1 concludes the proof.

\section{Grid graphs with uniform local structure and main spectral results}

This section is divided into two parts. In the first we give the definition of grid graphs with uniform local structure. In the second part we show the links of the above notions with Toeplitz and GLT sequences and we use the latter for proving the main spectral results. 


$$
\begin{aligned}
W & =\left(\begin{array}{llll}
0 & w & 0 & w \\
w & 0 & w & 0 \\
0 & w & 0 & w \\
w & 0 & w & 0
\end{array}\right) \\
L_{1} & =\left(\begin{array}{llll}
l_{11}^{1} & 0 & 0 & 0 \\
0 & 0 & 0 & 0 \\
0 & 0 & 0 & 0 \\
0 & 0 & 0 & 0
\end{array}\right) \\
L_{2} & =\left(\begin{array}{llll}
0 & 0 & 0 & 0 \\
0 & 0 & 0 & 0 \\
0 & 0 & l_{33}^{2} & 0 \\
0 & 0 & l_{43}^{2} & 0
\end{array}\right)
\end{aligned}
$$

$$
\left(\begin{array}{l|llllllllllllll}
\multicolumn{1}{c}{} & v_{1} & v_{2} & v_{3} & v_{4} & v_{5} & v_{6} & v_{7} & v_{8} & v_{9} & v_{10} & v_{11} & v_{12} \\
v_{1} & 0 & w & 0 & w & l_{11}^{1} & 0 & 0 & 0 & 0 & 0 & 0 & 0 \\
v_{2} & w & 0 & w & 0 & 0 & 0 & 0 & 0 & 0 & 0 & 0 & 0 \\
v_{3} & 0 & w & 0 & w & 0 & 0 & 0 & 0 & 0 & 0 & l_{33}^{2} & l_{43}^{2} \\
v_{4} & w & 0 & w & 0 & 0 & 0 & 0 & 0 & 0 & 0 & 0 & 0 \\
v_{5} & l_{11}^{1} & 0 & 0 & 0 & 0 & w & 0 & w & l_{11}^{1} & 0 & 0 & 0 \\
v_{6} & 0 & 0 & 0 & 0 & w & 0 & w & 0 & 0 & 0 & 0 & 0 \\
v_{7} & 0 & 0 & 0 & 0 & 0 & w & 0 & w & 0 & 0 & 0 & 0 \\
v_{8} & 0 & 0 & 0 & 0 & w & 0 & w & 0 & 0 & 0 & 0 & 0 \\
v_{9} & 0 & 0 & 0 & 0 & l_{11}^{1} & 0 & 0 & 0 & 0 & w & 0 & w \\
v_{10} & 0 & 0 & 0 & 0 & 0 & 0 & 0 & 0 & w & 0 & w & 0 \\
v_{11} & 0 & 0 & l_{33}^{2} & 0 & 0 & 0 & 0 & 0 & 0 & w & 0 & w \\
v_{12} & 0 & 0 & l_{43}^{2} & 0 & 0 & 0 & 0 & 0 & w & 0 & w & 0 \\
\hline
\end{array}\right.
$$

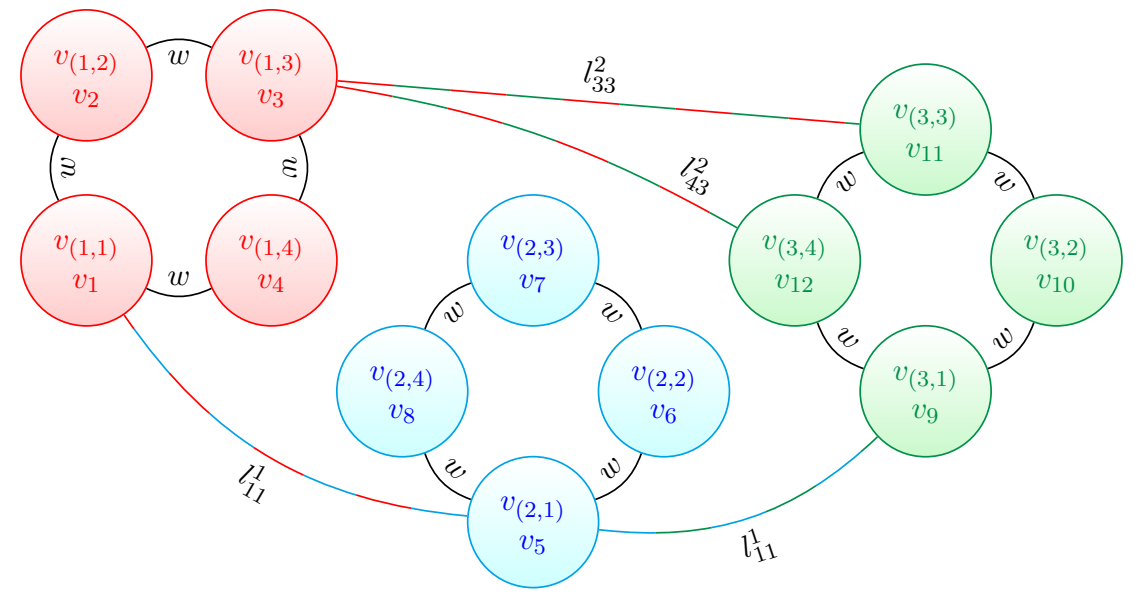

Figure 3. Example of a 1-level diamond Toeplitz graph $T_{n}^{G}\left\langle\left(1, L_{1}\right),\left(2, L_{2}\right)\right\rangle$, with $n=3$ and mold graph $G=T_{4}\langle(1, w),(3, w)\rangle$. The adjacency matrix of $G$ is $W$. The node sets of the diamond graphs $G(1), G(2), G(3)$ are $V(1)=\left\{v_{(1,1)}, v_{(1,2)}, v_{(1,3)}, v_{(1,4)}\right\}, V(2)=$ $\left\{v_{(2,1)}, v_{(2,2)}, v_{(2,3)}, v_{(2,4)}\right\}$ and $V(3)=\left\{v_{(3,1)}, v_{(3,2)}, v_{(3,3)}, v_{(3,4)}\right\}$, respectively. Clearly, all the diamond graphs are characterized by the same adjacency matrix $W$. The adjacency matrix $W_{n}$ of the whole graph is a 1-level block Toeplitz and has symbol function $\boldsymbol{f}(\theta)=W+2 L_{1} \cos (\theta)+\left(L_{2}+L_{2}^{*}\right) \cos (2 \theta)+\left(L_{2}-L_{2}^{*}\right) \mathrm{i} \sin (2 \theta)$.

\subsection{Sequence of grid graphs with uniform local structure}

The main idea in this section is to immerse the graphs presented in Section 2.2 inside a bounded regular domain $\Omega \subset \mathbb{R}^{d}$. We start with a series of definitions in order to give a mathematical rigor to our derivations. 
Definition 5.1 ( $d$-level Toeplitz grid graphs in the cube). Given a continuous almost everywhere (a.e.) function $p:[0,1]^{d} \rightarrow \mathbb{R}$, choose a $d$-level Toeplitz graph

$$
T_{\boldsymbol{n}}\left\langle\left\{\left[\boldsymbol{t}_{1}\right], \boldsymbol{w}_{1}\right\}, \ldots,\left\{\left[\boldsymbol{t}_{m}\right], \boldsymbol{w}_{m}\right\}\right\rangle,
$$

and consider the $d$-dimensional vector

$$
\boldsymbol{h}:=\left(h_{1}, \ldots, h_{d}\right)=\left(\frac{1}{n_{1}+1}, \ldots, \frac{1}{n_{d}+1}\right) .
$$

We introduce a bijective correspondence between the nodes $v_{j}$ of $T_{\boldsymbol{n}}\left\langle\left\{\left[\boldsymbol{t}_{1}\right], \boldsymbol{w}_{1}\right\}, \ldots,\left\{\left[\boldsymbol{t}_{m}\right], \boldsymbol{w}_{m}\right\}\right\rangle$ and the interior points $\boldsymbol{x}$ of the cube $[0,1]^{d}$ by the immersion map $\iota: V_{\boldsymbol{n}} \rightarrow(0,1)^{d}$ such that

$$
\iota\left(v_{\boldsymbol{j}}\right):=\boldsymbol{j} \circ \boldsymbol{h}=\left(j_{1} h_{1}, \ldots, j_{d} h_{d}\right)
$$

with $\circ$ being the Hadamard (component-wise) product. The $d$-level Toeplitz graph induces a grid graph in $[0,1]^{d}, G=\left(V_{\boldsymbol{n}}^{\prime}, E_{\boldsymbol{n}}^{\prime}, w^{p}\right)$ with

$$
V_{\boldsymbol{n}}^{\prime}:=\left\{\boldsymbol{x}_{\boldsymbol{k}}=\iota\left(v_{\boldsymbol{k}}\right) \mid \mathbf{1} \unlhd \boldsymbol{k} \unlhd \boldsymbol{n}\right\}, \quad E_{\boldsymbol{n}}^{\prime}:=\left\{\left(\boldsymbol{x}_{\boldsymbol{i}}, \boldsymbol{x}_{\boldsymbol{j}}\right) \mid w^{p}\left(\boldsymbol{x}_{\boldsymbol{i}}, \boldsymbol{x}_{\boldsymbol{j}}\right) \neq 0\right\},
$$

where

$$
w^{p}\left(\boldsymbol{x}_{\boldsymbol{i}}, \boldsymbol{x}_{\boldsymbol{j}}\right):=p\left(\frac{\boldsymbol{x}_{\boldsymbol{i}}+\boldsymbol{x}_{\boldsymbol{j}}}{2}\right) w\left(v_{\boldsymbol{i}}, v_{\boldsymbol{j}}\right),
$$

and $w$ is the weight function defined in (9). With abuse of notation we identify $V_{\boldsymbol{n}}=V_{\boldsymbol{n}}^{\prime}$ and we write

$$
T_{\boldsymbol{n}}\left\langle\left\{\left[\boldsymbol{t}_{1}\right], \boldsymbol{w}_{1}^{p}\right\}, \ldots,\left\{\left[\boldsymbol{t}_{m}\right], \boldsymbol{w}_{m}^{p}\right\}\right\rangle,
$$

for a $d$-level grid graph in $[0,1]^{d}$.

Observe that now $\boldsymbol{w}_{k}^{p}$, for $k=1, \ldots, m$, are not constant vectors as $\boldsymbol{w}_{k}$, but vector-valued functions $\boldsymbol{w}_{k}^{p}:[0,1]^{d} \times[0,1]^{d} \rightarrow \mathbb{R}^{c_{k}}$, with $c_{k}=\left|\left[\boldsymbol{t}_{k}\right]\right|$, such that

$$
\left(\boldsymbol{w}_{k}^{p}\right)_{\alpha}\left(\boldsymbol{x}_{\boldsymbol{i}}, \boldsymbol{x}_{\boldsymbol{j}}\right)= \begin{cases}p\left(\frac{\boldsymbol{x}_{\boldsymbol{i}}+\boldsymbol{x}_{\boldsymbol{j}}}{2}\right) w_{\left[\boldsymbol{t}_{k}\right]_{\alpha}^{+}} & \text {if }|\boldsymbol{i}-\boldsymbol{j}|=\boldsymbol{t}_{k} \\ & \text { and }(\boldsymbol{i}-\boldsymbol{j}) \in\left[\boldsymbol{t}_{k}\right]_{\alpha}=\left\{\left[\boldsymbol{t}_{k}\right]_{\alpha}^{+},\left[\boldsymbol{t}_{k}\right]_{\alpha}^{-}\right\} \\ 0 & \text { otherwise }\end{cases}
$$

for $\alpha=1, \ldots, c_{k}$. It is then not difficult to see that we can express the weight function $w^{p}$ as

$$
w^{p}\left(\boldsymbol{x}_{\boldsymbol{i}}, \boldsymbol{x}_{\boldsymbol{j}}\right)=\sum_{k=1}^{m} \sum_{\alpha=1}^{c_{k}}\left(\boldsymbol{w}_{k}^{p}\right)_{\alpha}\left(\boldsymbol{x}_{\boldsymbol{i}}, \boldsymbol{x}_{\boldsymbol{j}}\right) .
$$

In other words, taking in mind the role of the reference domain $[0,1]^{d}, \boldsymbol{x}_{\boldsymbol{i}}$ can be connected to $\boldsymbol{x}_{\boldsymbol{j}}$ only if $\left|\left(\boldsymbol{x}_{\boldsymbol{j}}\right)_{r}-\left(\boldsymbol{x}_{\boldsymbol{i}}\right)_{r}\right|=O\left(h_{r}\right)$, for all $r=1, \ldots, d$. From this property we derive the name of 'grid graphs with local structure'. Naturally, the above notion can be generalized to any domain $\Omega \subset[0,1]^{d}$ : as we see in the next subsection, the only restriction in order to have meaningful spectral properties of the related sequences, is that $\Omega$ is regular. 
Definition $5.2(d$-level Toeplitz grid graphs in $\Omega)$. Given a regular domain $\Omega \subseteq[0,1]^{d}$ and a continuous a.e. function $p:[0,1]^{d} \rightarrow \mathbb{R}$, choose a $d$-level Toeplitz graph

$$
T_{\boldsymbol{n}}\left\langle\left\{\left[\boldsymbol{t}_{1}\right], \boldsymbol{w}_{1}\right\}, \ldots,\left\{\left[\boldsymbol{t}_{m}\right], \boldsymbol{w}_{m}\right\}\right\rangle,
$$

and consider its associated $d$-level Toeplitz grid graph $T_{\boldsymbol{n}}\left\langle\left\{\left[\boldsymbol{t}_{1}\right], \boldsymbol{w}_{1}^{p}\right\}, \ldots\right.$, $\left.\left\{\left[\boldsymbol{t}_{m}\right], \boldsymbol{w}_{m}^{p}\right\}\right\rangle$. We define the $d$-level Toeplitz grid graph immersed in $\Omega$ as the graph $G=\left(V_{n^{\prime}}^{\Omega}, E_{n^{\prime}}^{\Omega}, w^{\Omega, p}\right)$ such that

$$
V_{n^{\prime}}^{\Omega}:=V_{\boldsymbol{n}} \cap \Omega, \quad w^{\Omega, p}:=w_{\mid V_{\boldsymbol{n}}^{\Omega} \times V_{\boldsymbol{n}}^{\Omega}}^{p} .
$$

Clearly, $\left|V_{n^{\prime}}^{\Omega}\right|=n^{\prime} \leq \prod_{r=1}^{d} n_{r}=\left|V_{\boldsymbol{n}}\right|$. Nevertheless, $n^{\prime}=n^{\prime}(\boldsymbol{n}) \rightarrow \infty$ as $\boldsymbol{n} \rightarrow \infty$. Therefore, with abuse of notation, we keep writing $\boldsymbol{n}$ instead of $n^{\prime}$. We indicate such a graph with the notation

$$
T_{\boldsymbol{n}}^{\Omega}\left\langle\left\{\left[\boldsymbol{t}_{1}\right], \boldsymbol{w}_{1}^{p}\right\}, \ldots,\left\{\left[\boldsymbol{t}_{m}\right], \boldsymbol{w}_{m}^{p}\right\}\right\rangle .
$$

In the application, as we see in Section 7 , once it is chosen the domain $\Omega$ and the kind of discretization technique to solve a differential equation, the weight function $w$ is fixed accordingly, and consequently the coefficients $\boldsymbol{w}_{1}, \ldots, \boldsymbol{w}_{m}$. In particular, it is important to remark that the weight function of $T_{\boldsymbol{n}}\left\langle\left\{\left[\boldsymbol{t}_{1}\right], \boldsymbol{w}_{1}\right\}, \ldots,\left\{\left[\boldsymbol{t}_{m}\right], \boldsymbol{w}_{m}\right\}\right\rangle$ depends on the differential equation and on the discretization technique.

Finally, we immerse the diamond graphs in the cube $[0,1]^{d}$ (and then in a generic regular domain $\left.\Omega \subset[0,1]^{d}\right)$.

Definition 5.3 ( $d$-level diamond Toeplitz grid graphs in the cube). The same definition as in Definition 5.1 where the $d$-level Toeplitz graph is replaced by a $d$-level diamond Toeplitz graph. The only difference now is that

$$
\boldsymbol{h}:=\left(h_{1}, \ldots, h_{d}\right)=\left(\frac{1}{\nu n_{1}+1}, \frac{1}{n_{2}+1}, \ldots, \frac{1}{n_{d}+1}\right),
$$

and

$$
\iota\left(v_{(\boldsymbol{j}, r)}\right):=(\boldsymbol{j}, r) \circ \boldsymbol{h}=\left(\left(j_{1}+r-1\right) h_{1}, j_{2} h_{2}, \ldots, j_{d} h_{d}\right), \quad r=1, \ldots, \nu .
$$

With abuse of notation we write

$$
T_{\boldsymbol{n}, \nu}^{G}\left\langle\left\{\left[\boldsymbol{t}_{1}\right], \boldsymbol{L}_{1}^{p}\right\}, \ldots,\left\{\left[\boldsymbol{t}_{m}\right], \boldsymbol{L}_{m}^{p}\right\}\right\rangle,
$$

for a $d$-level diamond Toeplitz grid graph in $[0,1]^{d}$.

While in the case of a $d$-level Toeplitz graph the immersion map $\iota$ was introduced naturally as the Hadamard product between the indices of the graph nodes and the natural Cartesian representation of points in $\mathbb{R}^{d}$, diamond Toeplitz graphs grant another degree of freedom for the immersion map. In Definition 5.3 we decided for the simplest choice, namely lining-up all the nodes of the diamonds along the first axis. Clearly, other choices of the immersion map $\iota$ would be able to describe more complex grid geometries. 
Definition 5.4 ( $d$-level diamond Toeplitz grid graphs in $\Omega$ ). The same definition as in Definition 5.2 where the $d$-level Toeplitz grid graph is replaced by a $d$-level diamond Toeplitz grid graph. We indicate such a graph with the notation

$$
T_{\boldsymbol{n}, \nu}^{G, \Omega}\left\langle\left\{\left[\boldsymbol{t}_{1}\right], \boldsymbol{L}_{1}^{p}\right\}, \ldots,\left\{\left[\boldsymbol{t}_{m}\right], \boldsymbol{L}_{m}^{p}\right\}\right\rangle .
$$

\subsection{Asymptotic spectral results}

We start this section, containing the spectral results, by giving the distribution theorem in the Weyl sense in its maximal generality, i.e. for a sequence of weighted (diamond) local grid graphs in $\Omega$, according to the case depicted in Definition 5.4.

Theorem 5.1. Given a regular domain $\Omega \subseteq[0,1]^{d}$ and a continuous a.e. function $p: \Omega \rightarrow \mathbb{R}$, fix a d-level Toeplitz grid graph

$$
T_{\boldsymbol{n}}^{\Omega}\left\langle\left\{\left[\boldsymbol{t}_{1}\right], \boldsymbol{w}_{1}^{p}\right\}, \ldots,\left\{\left[\boldsymbol{t}_{m}\right], \boldsymbol{w}_{m}^{p}\right\}\right\rangle
$$

as in Definition 5.2, and assume that $m,\left\{\left[\boldsymbol{t}_{1}\right], \boldsymbol{w}_{1}\right\}, \ldots,\left\{\left[\boldsymbol{t}_{m}\right], \boldsymbol{w}_{m}\right\}$ are fixed and independent of $\boldsymbol{n}$. Then, indicating with $\left\{W_{\boldsymbol{n}}^{\Omega, p}\right\}_{\boldsymbol{n}}$ the sequence of adjacency matrix of the d-level Toeplitz grid graph as $\boldsymbol{n} \rightarrow \infty$, it holds that

$$
\left\{W_{\boldsymbol{n}}^{\Omega, p}\right\}_{\boldsymbol{n}} \sim_{\lambda} \mathfrak{f}, \quad \mathfrak{f}: \Omega \times[-\pi, \pi]^{d} \subset \mathbb{R}^{2 d} \rightarrow \mathbb{R}
$$

and

$$
\mathfrak{f}(\boldsymbol{x}, \boldsymbol{\theta})=p(\boldsymbol{x}) f(\boldsymbol{\theta}),
$$

where $f(\boldsymbol{\theta})$ is the symbol function defined in (11).

Proof. We note that, in the case where $\nu=1$, a $d$-level diamond Toeplitz grid graph reduces to a $d$-level Toeplitz grid graph according to Definition 5.2. The conclusion of the theorem is then obvious once we prove our next result, Theorem 5.2.

Theorem 5.2. Given a regular domain $\Omega \subseteq[0,1]^{d}$ and a continuous a.e. function $p: \Omega \rightarrow \mathbb{R}$, fix a d-level diamond Toeplitz grid graph

$$
T_{\boldsymbol{n}, \nu}^{G, \Omega}\left\langle\left\{\left[\boldsymbol{t}_{1}\right], \boldsymbol{L}_{1}^{p}\right\}, \ldots,\left\{\left[\boldsymbol{t}_{m}\right], \boldsymbol{L}_{m}^{p}\right\}\right\rangle
$$

as in Definition 5.4, and assume that $m,\left\{\left[\boldsymbol{t}_{1}\right], \boldsymbol{L}_{1}\right\}, \ldots,\left\{\left[\boldsymbol{t}_{m}\right], \boldsymbol{L}_{m}\right\}$ are fixed and independent of $\boldsymbol{n}$. Then, indicating with $\left\{W_{\boldsymbol{n}, \nu}^{G, \Omega, p}\right\}_{\boldsymbol{n}}$ the sequence of adjacency matrix of the d-level diamond Toeplitz grid graph as $\boldsymbol{n} \rightarrow \infty$, it holds that

$$
\left\{W_{\boldsymbol{n}, \nu}^{G, \Omega, p}\right\}_{\boldsymbol{n}} \sim_{\lambda} \mathfrak{f}, \quad \mathfrak{f}: \Omega \times[-\pi, \pi]^{d} \subset \mathbb{R}^{2 d} \rightarrow \mathbb{C}^{\nu \times \nu}
$$

with $\mathfrak{f}$ a matrix-valued function and

$$
\mathfrak{f}(\boldsymbol{x}, \boldsymbol{\theta})=p(\boldsymbol{x}) \boldsymbol{f}(\boldsymbol{\theta}),
$$

where $\boldsymbol{f}(\boldsymbol{\theta})$ is the symbol function defined in (12).

Proof. First of all we observe that our assumption of $\Omega$ regular is equivalent to require $\Omega$ to be measurable according to Peano-Jordan, which is the fundamental assumption to apply the GLT theory (see [23]).

Assume $\Omega=[0,1]^{d}$ and $p(x) \equiv 1$ over $\Omega$. Then our sequence of graphs reduces to a sequence of $d$-level diamond Toeplitz graphs and the proof is over using Proposition 4.4 . 
Assuming now that $\Omega=[0,1]^{d}$ and $p$ is just a Riemann-integrable function over $\Omega$, we decompose the adjacency matrix $W_{\boldsymbol{n}, \nu}^{G, p}$ as $W_{\boldsymbol{n}, \nu}^{G, p}=\operatorname{diag}_{\boldsymbol{n}}(p) T_{\boldsymbol{n}}(\boldsymbol{f})+E_{\boldsymbol{n}}$. The only observation needed here is that $\left\{\operatorname{diag}_{\boldsymbol{n}}(p)\right\}$ is a multilevel block GLT with symbol function $p$, while $\left\{T_{\boldsymbol{n}}(\boldsymbol{f})\right\}$ is a multilevel block GLT with symbol function $\boldsymbol{f}$ (see item (GLT 3)). Moreover, by direct calculation, we see that $E_{\boldsymbol{n}}$, for $\boldsymbol{n}$ large, can be written as a term of small spectral norm, plus a term of relatively small rank. Therefore, $E_{\boldsymbol{n}}$ is a zero-distributed sequence of matrices and hence a multilevel block GLT with symbol function 0. Summing up we have

$$
\left\{T_{\boldsymbol{n}}(\boldsymbol{f})\right\}_{\boldsymbol{n}} \sim_{\mathrm{GLT}} \boldsymbol{f} \text { over }[-\pi, \pi]^{d},\left\{\operatorname{diag}_{\boldsymbol{n}}(\mathrm{p})\right\}_{\boldsymbol{n}} \sim_{\mathrm{GLT}} p \text { over } \Omega,\left\{E_{\boldsymbol{n}}\right\}_{\boldsymbol{n}} \sim_{\mathrm{GLT}} 0 .
$$

Now, by the structure of algebra of multilevel block GLT sequences and using the symmetry of the sequence (see item (GLT 1) and (GLT 4)), we conclude that $\left\{W_{\boldsymbol{n}, \nu}^{G, p}\right\}_{\boldsymbol{n}} \sim_{\lambda} p(\boldsymbol{x}) \boldsymbol{f}(\boldsymbol{\theta})$ over $[0,1]^{d} \times[-\pi, \pi]^{d}$.

For the general case where $\Omega$ is a generic regular subset of $[0,1]^{d}$, we simply notice that, using Definition 5.4, we can see $W_{\boldsymbol{n}, \nu}^{G, p}$ as a principal sub-matrix of $W_{\boldsymbol{n}, \nu}^{G, \Omega, p}$, where $\left\{W_{\boldsymbol{n}, \nu}^{G, \Omega, p}\right\}$ is constructed according to Definition 5.3 and the function $p$ is substituted by $p_{\mid \Omega}:=p(i(\boldsymbol{x}))$, where $i: \Omega \hookrightarrow[0,1]^{d}$ is the inclusion map. Since $\Omega$ is regular we conclude that $p_{\mid \Omega}$ is Riemann-integrable, $p_{\mid \Omega} \equiv p$ over $\Omega$, and that

$$
\left\{W_{\boldsymbol{n}, \nu}^{G, \Omega, p}\right\}_{\boldsymbol{n}} \sim_{\mathrm{GLT}} p(\boldsymbol{x}) \boldsymbol{f}(\boldsymbol{\theta}),
$$

with $p(\boldsymbol{x}) \boldsymbol{f}(\boldsymbol{\theta})$ defined over $\Omega \times[-\pi, \pi]^{d}$, which implies the desired conclusion.

Corollary 5.3. Let $G_{\boldsymbol{n}, \nu}=\left(T_{\boldsymbol{n}, \nu}^{G, \Omega}\left\langle\left(\left[\boldsymbol{t}_{1}\right], \boldsymbol{L}_{1}^{p}\right), \ldots,\left(\left[\boldsymbol{t}_{m}\right], \boldsymbol{L}_{m}^{p}\right)\right\rangle, \kappa_{n}\right)$ be a d-level diamond Toeplitz grid graph as in Definition 5.4, plus a potential term $\kappa_{n}: V_{\boldsymbol{n}} \rightarrow[0, \infty)$. Let $D, K$ be as in Definition 2.2. If

$$
D+K=c I \cdot \operatorname{diag}_{(\mathbf{1}, 1) \unlhd(\boldsymbol{i}, r) \unlhd(\boldsymbol{n}, \nu)}\left\{p\left(\boldsymbol{x}_{(\boldsymbol{i}, r)}\right)\right\}+o(\mathbf{1}),
$$

where $c \in \mathbb{R}$ is a fixed constant, $I$ is the identity matrix and $p: \Omega \subset[0,1]^{d} \rightarrow \mathbb{R}$ is a continuous a.e. function as in Definition 5.4, then it holds that

$$
\left\{\Delta_{G_{\boldsymbol{n}, \nu}}\right\} \sim_{\lambda} \mathfrak{f}(\boldsymbol{x}, \boldsymbol{\theta})=p(\boldsymbol{x})(c I-\boldsymbol{f}(\boldsymbol{\theta})), \quad(\boldsymbol{x}, \boldsymbol{\theta}) \in \Omega \times[-\pi, \pi]^{d},
$$

where $\Delta_{G_{\boldsymbol{n}, \nu}}$ is the graph-Laplacian as in Definition 2.2 and $\boldsymbol{f}(\boldsymbol{\theta})$ is defined in (12).

Proof. From Definition 2.2, $\Delta_{G_{n, \nu}}=D+K-W_{n, \nu}^{G, \Omega, p}$. By assumption, note that $D+K$ is a GLT sequence with symbol function $c p$, so that the conclusion follows once again by the algebra structure of the GLT sequences.

\section{Spectral gaps}

In this section we report general results on the gaps between the extreme eigenvalues of adjacency and graph-Laplacian matrices of the type considered so far. We 
recall that the study of the spectral gaps has applications in several directions including the algebraic connectivity, the theory of the consensus among multi-agents, the superdiffusivity etc. (see e.g. [7, 16, 17, 18, 19, 20]).

The spectral properties of a Toeplitz matrix $T_{n}(f)$ are well understood by considering $f$; in fact, it is known (see e.g. [29, 30]) that the spectrum of $T_{n}(f)$ is contained in $\left(m_{f}, M_{f}\right)$, where $m_{f}=\min f$ and $M_{f}=\max f$, and moreover

$$
\lim _{n \rightarrow \infty} \lambda_{1}^{(n)}=m_{f}
$$

and

$$
\lim _{n \rightarrow \infty} \lambda_{n}^{(n)}=M_{f}
$$

Recall the following result due to Kac, Murdoch, Szegö, Parter, Widom in the early fifties and, later, to Serra-Capizzano, Böttcher, Grudsky (see also [5] and references therein for more details and for the history of such results).

Theorem 6.1. Let $f \in C_{2 \pi}[-\pi, \pi]$ be a continuous function on $[-\pi, \pi]$ extended periodically on $\mathbb{R}$, let $m_{f}=\min f$ and $M_{f}=\max f$. Let $T_{n}(f)$ be the associated Toeplitz matrix and let

$$
\lambda_{1}^{(n)} \leq \lambda_{2}^{(n)} \leq \ldots \leq \lambda_{n-1}^{(n)} \leq \lambda_{n}^{(n)}
$$

be the eigenvalues of $T_{n}(f)$ ordered in non-decreasing order.

Then, for all fixed $j$ we have that

$$
\lim _{n \rightarrow \infty} \lambda_{j}^{(n)}=m_{f} \quad \lim _{n \rightarrow \infty} \lambda_{n+1-j}^{(n)}=M_{f}
$$

and, moreover,

$$
\lim _{n \rightarrow \infty} \frac{M_{f}-\lambda_{n+1-j}^{(n)}}{c\left(j^{-1} n\right)^{\alpha}}=1, \quad c \in \mathbb{R}
$$

where $\alpha \in \mathbb{R}$ is such that, if $f\left(x_{0}\right)=M_{f},\left|f\left(x_{0}\right)-f(x)\right| \sim c\left|x-x_{0}\right|^{\alpha}$ as $x \rightarrow x_{0}$.

Thanks to the results reported in Section 3, we can make the following statements concerning spectral properties of $d$-level diamond Toeplitz (grid) graphs.

Corollary 6.2. Let $\left\{W_{\boldsymbol{n}, \nu}^{G, \Omega, p}\right\}_{\boldsymbol{n}}$ be a sequence of adjacency matrices of d-level diamond Toeplitz grid graphs as in Theorem 5.2, with $\left\{\lambda_{k}^{(\boldsymbol{n})}\right\}_{k=1}^{d_{\boldsymbol{n}}}$ the collection of their eigenvalues sorted in non-decreasing order and where $d_{\boldsymbol{n}}=\nu \prod_{j=1}^{d} n_{j}$ is the dimension of the matrices. Let $p(\boldsymbol{x})$ be piecewise continuous and let $\mathfrak{f}^{\dagger}:[0,1] \rightarrow\left[\min R_{\mathfrak{f}}, \max R_{\mathfrak{f}}\right]$ be the monotone rearrangement of the symbol function $\mathfrak{f}(\boldsymbol{x}, \boldsymbol{\theta})=p(\boldsymbol{x}) \boldsymbol{f}(\boldsymbol{\theta})$ of $\left\{W_{\boldsymbol{n}, \nu}^{G, \Omega, p}\right\}$, as in Definition 3.1. Finally, let $\tau:\left[\min R_{\mathfrak{f}}, \max R_{\mathfrak{f}}\right] \rightarrow \mathbb{R}$ be a function that is differentiable in $\max R_{\mathfrak{f}}$ and define $x_{n}:=1-\frac{1}{d_{n}}$. If

(i) $\lambda_{d_{\boldsymbol{n}}-1}^{(\boldsymbol{n})}<\lambda_{d_{\boldsymbol{n}}}^{(\boldsymbol{n})} \leq \max R_{\mathfrak{f}}$ definitely for $\boldsymbol{n} \rightarrow \infty$,

(ii) $\mathfrak{f}^{\dagger}$ is differentiable at $x=1$ with $\mathfrak{f}^{\dagger}(1) \neq 0$,

then it holds that

$$
\lim _{\boldsymbol{n} \rightarrow \infty} d_{\boldsymbol{n}}\left[\tau\left(\lambda_{d_{\boldsymbol{n}}}^{(\boldsymbol{n})}\right)-\tau\left(\lambda_{d_{\boldsymbol{n}}-1}^{(\boldsymbol{n})}\right)\right]=\lim _{\boldsymbol{n} \rightarrow \infty} \tau^{\prime}\left(\mathfrak{f}\left(x_{\boldsymbol{n}}\right)\right) \mathfrak{f}^{\prime}\left(x_{\boldsymbol{n}}\right)
$$


Proof. From Lemma 3.1 we have that

$$
\left\{W_{\boldsymbol{n}, \nu}^{G, \Omega, p}\right\}_{\boldsymbol{n}} \sim_{\lambda} \mathfrak{f}(\boldsymbol{x}, \boldsymbol{\theta})=p(\boldsymbol{x}) \sum_{k=1}^{\nu} \lambda_{k}\left(\boldsymbol{f}_{k}(\boldsymbol{\theta})\right),
$$

where $\boldsymbol{f}$ is defined in (12) and consequently $\boldsymbol{f}_{k}$ in Lemma 3.1. Therefore it is easy to check that

$$
\mu_{m}\left(\left\{(\boldsymbol{x}, \boldsymbol{\theta}) \in \bar{\Omega} \times[-\pi, \pi]^{d}: p(\boldsymbol{x}) \sum_{k=1}^{\nu} \lambda_{k}\left(\boldsymbol{f}_{k}(\boldsymbol{\theta})\right)=t\right\}\right)=0 \quad \forall t \in R_{\mathfrak{f}},
$$

and then all the hypothesis of Theorem 3.2 are satisfied. Because of (i)-(ii), it holds (7) at $x_{0}=1$ and we can then apply the same proof of Corollary 3.4.

Corollary 6.3. In the case $p(\boldsymbol{x}) \equiv c$ and $d=1$, then $\mathfrak{f}^{\dagger}$ is differentiable almost everywhere in $[0,1]$ and

$$
\left(\mathfrak{f}^{\dagger}\right)^{\prime}(1)=0
$$

for any choice of $\left\{\left(t_{1}, L_{t_{1}}\right), \ldots,\left(t_{m}, L_{t_{m}}\right)\right\}$ (or $\left\{\left(t_{1}, w_{t_{1}}\right), \ldots,\left(t_{m}, w_{t_{m}}\right)\right\}$ in the case $\nu=1)$. In particular, if $\tau \equiv$ id and $\mathfrak{f}^{\dagger}(1) \neq 0$, then

$$
\nu n\left[\lambda_{\nu n}^{(n)}-\lambda_{\nu n-1}^{(n)}\right] \rightarrow 0 \quad \text { as } n \rightarrow \infty .
$$

Proof. Let us fix $c=1$. Since all the components $f_{i, j}(\theta)$ of $\boldsymbol{f}(\theta)$ from (12) are analytic with $\boldsymbol{f}(\theta)$ Hermitian for every $\theta \in[-\pi, \pi]$, then there exists an ordering (not necessarily the usual ordering by increasing magnitude) such that $\hat{\lambda}_{k}(\boldsymbol{f}(\theta))$ are analytic real-valued functions for every $k=1, \ldots, \nu$ (see e.g. [31, Chapter 2]). Clearly, this kind of ordering does not affect the validity of Lemma 3.1. In particular, the function $\mathfrak{f}(\theta):=\sum_{k=1}^{\nu} \hat{\lambda}_{k}\left(\boldsymbol{f}_{k}(\theta)\right)$, as in Definition 3.1 is piecewise analytical: it can have a finite number of jumps at $\theta_{k}=\frac{(2 k-\nu) \pi}{\nu}$ for $k=1, \ldots, \nu-1$, and so its image set $R_{\mathrm{f}}$ can be a finite union of disjoint closed intervals. Then, by an appropriate modification of [32, Lemma 2.3], the monotone rearrangement $\mathfrak{f}^{\dagger}$ is piecewise Lipschitz continuous. Clearly, $\mathfrak{f}^{\dagger}$ is differentiable almost everywhere on $[0,1]$ and it is differentiable in $x=1$ iff $\lim _{x \rightarrow 1}\left(\mathfrak{f}^{\dagger}\right)^{\prime}(x)<\infty$. Let us prove then that $\lim _{x \rightarrow 1}\left(\mathfrak{f}^{\dagger}\right)^{\prime}(x)=0$. Suppose for the moment that there exists only one interior point $\theta_{M} \in(-\pi, \pi), \theta_{M} \neq \theta_{k}$, where $\mathfrak{f}$ achieves its maximum $M$, i.e., such that $\mathfrak{f}\left(\theta_{M}\right)=M=\max _{\theta \in[-\pi, \pi]} \mathfrak{f}(\theta)=\mathfrak{f}^{\dagger}(1)$. By regularity of $\mathfrak{f}$, there exists then $\delta>0$ such that $\mathfrak{f}$ is invertible on $I_{l}=\left(\theta_{M}-\delta, \theta_{M}\right.$ ] and $I_{r}=\left[\theta_{M}, \theta_{M}+\delta\right)$. By equations (5a)-(5b), there exists $\epsilon=\epsilon(\delta)$ such that

$$
\begin{aligned}
\phi(t) & =\phi(M-\epsilon)+\mathfrak{f}_{\mid I_{l}}^{-1}(t)+\mathfrak{f}_{\mid I_{r}}^{-1}(t) \\
& =\phi(M-\epsilon)+\left(\hat{\lambda}_{k} \circ \boldsymbol{f}_{k}\right)_{\mid I_{l}}^{-1}(t)+\left(\hat{\lambda}_{k} \circ \boldsymbol{f}_{k}\right)_{\mid I_{r}}^{-1}(t)
\end{aligned}
$$

for every $t \in[M-\epsilon, M]$, for a $k=1, \ldots, \nu$, and then,

$$
\left(\mathfrak{f}^{\dagger}\right)^{\prime}(x)=\frac{1}{\left[\left(\mathfrak{f}^{-1}\right)_{\mid I_{l}}^{\prime}(t)+\left(\mathfrak{f}^{-1}\right)_{\mid I_{r}}^{\prime}(t)\right]_{\mid t=\mathfrak{f}^{\dagger}(x)}}
$$


for every $x \in(1-\eta, 1]$ with $\eta=\eta(\epsilon)$ small enough. Since $t \rightarrow M$ as $x \rightarrow 1$ and $\mathfrak{f}_{\mid I_{l}}^{\prime}\left(\theta_{M}\right)=\mathfrak{f}_{\mid I_{r}}^{\prime}\left(\theta_{M}\right)=\mathfrak{f}^{\prime}\left(\theta_{M}\right)=0$, it follows that $\left(\mathfrak{f}^{\dagger}\right)^{\prime}(1)=0$. In the case that $\theta_{M}=\theta_{k}$ or $\theta_{M}= \pm \pi$, then

$$
\mathfrak{f}_{\mid I_{l}}^{-1}(t)=\left(\hat{\lambda}_{k} \circ \boldsymbol{f}_{k}\right)_{\mid I_{l}}^{-1}(t), \quad \mathfrak{f}_{\mid I_{r}}^{-1}(t)=\left(\hat{\lambda}_{k+1} \circ \boldsymbol{f}_{k+1}\right)_{\mid I_{r}}^{-1}(t),
$$

and we can conclude again that $\left(\mathfrak{f}^{\dagger}\right)^{\prime}(1)=0$ if we show that

$$
\left[\hat{\lambda}_{k}\left(\boldsymbol{f}_{k}\left(\theta_{k}\right)\right)\right]^{\prime}=\left[\hat{\lambda}_{k}(\boldsymbol{f}(\pi))\right]^{\prime}=0=\left[\hat{\lambda}_{k+1}(\boldsymbol{f}(-\pi))\right]^{\prime}=\left[\hat{\lambda}_{k+1}\left(\boldsymbol{f}_{k+1}\left(\theta_{k}\right)\right)\right]^{\prime} .
$$

Due to the peculiar structure of the matrix function $\boldsymbol{f}$ from (12), for $d=1$, by direct computation it can be shown that the coefficients of its characteristic polynomial $\chi(\lambda)$ are functions of $\left(\cos \left(t_{1} \theta\right), \ldots, \cos \left(t_{m} \theta\right)\right)$. Therefore, $\hat{\lambda}_{k}(\boldsymbol{f}(\theta))=$ $h_{k}\left(\left(\cos \left(t_{1} \theta\right), \ldots, \cos \left(t_{m} \theta\right)\right)\right)$ with $h_{k}$ an analytic function, and then it follows easily that $\left[\hat{\lambda}_{k}(\boldsymbol{f}( \pm \pi))\right]^{\prime}=0$. The generalization to the case of existence of countable many points $\theta_{M, j}$ such that $g\left(\theta_{M, j}\right)=M$ is straightforward.

Finally, to prove (13) we suppose $\lambda_{\nu n}^{(n)}>\lambda_{\nu n-1}^{(n)}$, otherwise the thesis would be trivial. By [36, Theorem 2.2] we have that $\lambda_{\nu n}^{(n)} \leq \max _{\theta \in[-\pi, \pi]} \mathfrak{f}(\theta)$. Therefore all the hypotheses of Corollary 6.2 are satisfied and the thesis follows at once.

In general, $\mathfrak{f}^{\dagger}$ is absolute continuous which means that it is a.e. differentiable. Therefore, it is differentiable in $x=1$ iff $\sup _{x \in[0,1]}\left(\mathfrak{f}^{\dagger}\right)^{\prime}(x)<\infty$. If it happens that $\lim _{x \rightarrow 1}\left(\mathfrak{f}^{\dagger}\right)^{\prime}(x)=\infty$, then $d_{\boldsymbol{n}}\left(\lambda_{d_{\boldsymbol{n}}}^{(\boldsymbol{n})}-\lambda_{d_{\boldsymbol{n}}-1}^{(\boldsymbol{n})}\right) \rightarrow \infty$ but it could diverge at a different rate with respect to $\left(\mathfrak{f}^{\dagger}\right)^{\prime}\left(x_{\boldsymbol{n}}\right)$.

\subsection{Examples}

Example 1. As a first example we consider the Toeplitz graph

$$
T_{n}\langle(1,1),(2,-6),(3,1),(4,1)\rangle
$$

with corresponding symbol function

$$
\mathfrak{f}(\boldsymbol{x}, \boldsymbol{\theta})=f(\theta)=2 \cos (\theta)-12 \cos (2 \theta)+2 \cos (3 \theta)+2 \cos (4 \theta),
$$

according to Proposition 4.2. In this case, by symmetry of the symbol $f$ over $[-\pi, \pi]$ we can restrict it to $\theta \in[0, \pi]$ without affecting the validity of the identity $(3)$. It is easy to verify that the graph is connected and that $\max _{[0, \pi]} f(\theta)=f^{\dagger}(1)>0$. In Figure 4 and Table 1 it is possible to check the numerical validity of Corollary 6.2, Corollary 6.3 and Theorem 3.2.

Example 2. For this example we consider a 2-level Toeplitz graph on $[0,1]^{2}$ given by

$$
T_{\boldsymbol{n}}\left\langle\left\{\left[\boldsymbol{t}_{1}\right], \boldsymbol{w}_{1}\right\},\left\{\left[\boldsymbol{t}_{2}\right], \boldsymbol{w}_{2}^{p}\right\},\left\{\left[\boldsymbol{t}_{3}\right], \boldsymbol{w}_{3}\right\},\left\{\left[\boldsymbol{t}_{4}\right], \boldsymbol{w}_{4}\right\}\right\rangle,
$$

like in Definition 5.1. We set $\boldsymbol{n}=(n, n)$ and

$$
\begin{array}{ll}
{\left[\boldsymbol{t}_{1}\right]=[(1,0)]=\left\{[(1,0)]_{1}\right\},} & {\left[\boldsymbol{t}_{2}\right]=[(0,1)]=\left\{[(0,1)]_{1}\right\},} \\
{\left[\boldsymbol{t}_{3}\right]=[(1,1)]=\left\{[(1,1)]_{1},[(1,1)]_{2}\right\},} & {\left[\boldsymbol{t}_{4}\right]=[(2,2)]=\left\{[(2,2)]_{1},[(2,2)]_{2}\right\},}
\end{array}
$$




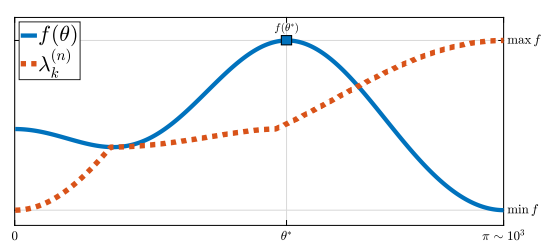

(A) Comparison between the distributions of $f$ and $\lambda_{k}^{(n)}$

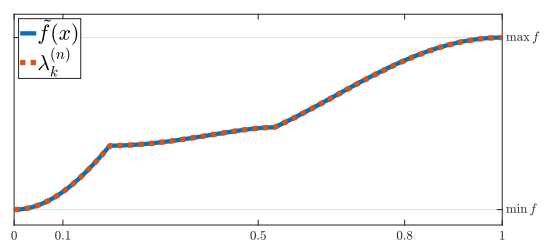

(в) Comparison between the distributions of $f^{\dagger}$ and $\lambda_{k}^{(n)}$

Figure 4. In Figure 4a we compare the distributions of $f(\theta)$, for $\theta \in$ $[0, \pi]$ uniformly sampled over $10^{3}$ sampling points, and of the eigenvalues $\lambda_{k}^{(n)}$ of the adjacency matrix of $T_{n}\langle(1,1),(2,-6),(3,1),(4,1)\rangle$, for $k=1, \ldots, 10^{3}$. With the notation of Definition 3.1, notice that $\lambda_{k}^{(n)} \in\left[\min R_{f}, \max R_{f}\right]=\left[\min _{[0, \pi]} f(\theta), \max _{[0, \pi]} f(\theta)\right]$. In Figure $4 \mathrm{~b}$, instead, it is possible to observe the validity of Theorem 3.2, comparing the distribution of an approximation of the monotone rearrangement $f^{\dagger}$, for $x \in[0,1]$, and the distribution of $\lambda_{k}^{(n)}$, for $k(n) / n \in[0,1]$ and $n=10^{3}$.

\begin{tabular}{|c|c|c|c|c|c|}
\hline & \multicolumn{4}{|c|}{ relative errors } \\
\hline & & $n=10^{2}$ & $n=5 \cdot 10^{2}$ & $n=10^{3}$ & $n=2 \cdot 10^{3}$ \\
\hline \multirow{4}{*}{$\frac{k(n)}{n}$} & 0.1 & 0.0039 & $8.1567 \mathrm{e}-04$ & $4.0990 \mathrm{e}-04$ & $2.0547 \mathrm{e}-04$ \\
\hline & 0.5 & 0.0013 & $6.7743 \mathrm{e}-04$ & 0.0025 & $1.0263 \mathrm{e}-04$ \\
\hline & 0.8 & 0.0502 & 0.0097 & 0.0035 & 0.0019 \\
\hline & 1 & 0.0028 & $1.1539 \mathrm{e}-04$ & $3.0532 \mathrm{e}-05$ & $6.1804 \mathrm{e}-06$ \\
\hline \multicolumn{2}{|c|}{$\bar{~} n\left[\lambda_{n}^{(n)}-\lambda_{n-1}^{(n)}\right]$} & 0.1662 & 0.0045 & 0.0019 & $2.0245 \mathrm{e}-04$ \\
\hline
\end{tabular}

TABLE 1. The first four rows present the relative errors between the eigenvalue $\lambda_{k(n)}^{(n)}$ and the sampling of the monotone rearrangement $f^{\dagger}\left(\frac{k(n)}{n}\right)$, i.e.: $\left|\frac{\lambda_{k(n)}^{(n)}}{f^{\dagger}(k(n) / n)}-1\right|$. The index $k(n)$ of the eigenvalue $\lambda_{k(n)}^{(n)}$ is chosen such that $k(n) / n$ is constant for every fixed $n=10^{2}, 5$. $10^{2}, 10^{3}, 2 \cdot 10^{3}$. As it can be seen, the relative errors decrease as $n$ increases, in accordance with Theorem 3.2. The convergence to zero is not uniform: one of the reasons is that we are using a linear approximation of $f^{\dagger}$ instead of $f^{\dagger}$ itself. In the last row we show the computation of the gap between the biggest and the second-biggest eigenvalues, confirming the prediction of Corollary 6.3.

where

$$
\begin{array}{ll}
{[(1,0)]_{1}=\{ \pm(1,0)\},} & {[(0,1)]_{1}=\{ \pm(0,1)\},} \\
{[(1,1)]_{1}=\{ \pm(1,-1)\},} & {[(1,1)]_{2}=\{ \pm(1,1)\},} \\
{[(2,2)]_{1}=\{ \pm(2,-2)\},} & {[(2,2)]_{2}=\{ \pm(2,2)\} .}
\end{array}
$$


Finally,

$$
\begin{array}{ll}
\boldsymbol{w}_{1}=w_{1,0}=1, & \boldsymbol{w}_{2}=w_{0,1}=2, \\
\boldsymbol{w}_{3}=\left(w_{1,-1}, w_{1,1}\right)=(-3,-3), & \boldsymbol{w}_{4}=\left(w_{2,-2}, w_{2,2}\right)=(1,1) .
\end{array}
$$

By Theorem 5.1, the sequence of adjacency matrices $\left\{W_{\boldsymbol{n}}\right\}_{\boldsymbol{n}}$ has symbol

$$
\begin{aligned}
\mathfrak{f}(\boldsymbol{x}, \boldsymbol{\theta})= & f\left(\theta_{1}, \theta_{2}\right) \\
= & 2 \cos \left(\theta_{1}\right)+4 \cos \left(\theta_{2}\right)-6 \cos \left(\theta_{1}-\theta_{2}\right)-6 \cos \left(\theta_{1}+\theta_{2}\right) \\
& +2 \cos \left(2 \theta_{1}-2 \theta_{2}\right)+2 \cos \left(2 \theta_{1}+2 \theta_{2}\right),
\end{aligned}
$$

with $\left(\theta_{1}, \theta_{2}\right) \in[-\pi, \pi]^{2}$ and $\max _{[-\pi, \pi]^{2}} f\left(\theta_{1}, \theta_{2}\right)=f^{\dagger}(1)>0$. Due to the symmetry of the symbol $f$ over $[-\pi, \pi]^{2}$ we can restrict it to $\left(\theta_{1}, \theta_{2}\right) \in[0, \pi]^{2}$ without affecting the validity of the identity (3). In Table 2 and Figure 5 it is possible to check numerically the validity of Theorem 3.2 and Corollary 6.2.

Example 3. We consider a sequence of adjacency matrices $\left\{W_{n}^{G}\right\}_{n}$ from the 1-level diamond Toeplitz graph given in Figure 3, namely $T_{n}^{G}\left\langle\left(1, L_{1}\right),\left(2, L_{2}\right)\right\rangle$ with mold graph $G=T_{4}\langle(1,1),(3,1)\rangle$ and

$$
\begin{gathered}
W=\left(\begin{array}{llll}
0 & 1 & 0 & 1 \\
1 & 0 & 1 & 0 \\
0 & 1 & 0 & 1 \\
1 & 0 & 1 & 0
\end{array}\right) \quad L_{1}=\left(\begin{array}{cccc}
-2 & 0 & 0 & 0 \\
0 & 0 & 0 & 0 \\
0 & 0 & 0 & 0 \\
0 & 0 & 0 & 0
\end{array}\right) \\
L_{2}=\left(\begin{array}{llll}
0 & 0 & 0 & 0 \\
0 & 0 & 0 & 0 \\
0 & 0 & \frac{1}{2} & 0 \\
0 & 0 & 6 & 0
\end{array}\right),
\end{gathered}
$$

where $W$ is the adjacency matrix of the mold graph $G$. From Proposition 4.4, $\left\{W_{n}^{G}\right\}_{n} \sim_{\lambda} \mathfrak{f}(\boldsymbol{x}, \boldsymbol{\theta})=\boldsymbol{f}(\theta)$, where

$$
\boldsymbol{f}(\theta)=W+2 L_{1} \cos (\theta)+\left(L_{2}+L_{2}^{*}\right) \cos (2 \theta)+\left(L_{2}-L_{2}^{*}\right) \mathrm{i} \sin (2 \theta), \theta \in[-\pi, \pi] .
$$

In this case, by symmetry of the symbol $\boldsymbol{f}$ over $[-\pi, \pi]$ we can restrict it to $\theta \in[0, \pi]$ without affecting the validity of the identity (3). Moreover, due to Lemma 3.1 and taking into account that we restricted $\theta$ to $[0, \pi]$, we write

$$
f(\theta)=\sum_{k=1}^{4} \mathbb{1}_{I_{k}}(\theta) \lambda_{k}\left(\boldsymbol{f}_{k}(\theta)\right),
$$

where

$$
\boldsymbol{f}_{k}(\theta)=\boldsymbol{f}(4 \theta-(k-1) \pi) \quad I_{k}=\left[\frac{(k-1) \pi}{4}, \frac{k \pi}{4}\right]
$$




\begin{tabular}{|c|c|c|c|c|}
\hline & \multicolumn{3}{|c|}{ relative errors } \\
\hline & & $n=10$ & $n=50$ & $n=100$ \\
\hline \multirow{4}{*}{$\frac{k(\boldsymbol{n})}{d_{n}}$} & 0.2 & 0.0422 & 0.0053 & 0.0029 \\
\hline & 0.5 & 0.2553 & 0.1211 & 0.0705 \\
\hline & 0.7 & 0.0396 & 0.0096 & 0.0089 \\
\hline & 1 & 0.0071 & $7.8515 \mathrm{e}-05$ & $1.0184 \mathrm{e}-05$ \\
\hline \multicolumn{2}{|c|}{$\left|\frac{\lambda_{d \boldsymbol{n}}^{(\boldsymbol{n})}-\lambda_{d \boldsymbol{n}-1}^{(\boldsymbol{n})}}{\mid f^{\dagger}(1)-f^{\dagger}\left(1-1 / d_{\boldsymbol{n}}\right)-1}\right|$} & 0.0487 & 0.0145 & 0.0075 \\
\hline
\end{tabular}

TABLE 2. The first four rows present the relative errors between the eigenvalue $\lambda_{k(\boldsymbol{n})}^{(\boldsymbol{n})}$ and the sampling of the monotone rearrangement $f^{\dagger}\left(\frac{k(\boldsymbol{n})}{d_{\boldsymbol{n}}}\right)$. The index $k(\boldsymbol{n})$ is chosen such that $k(\boldsymbol{n}) / d_{\boldsymbol{n}}$ is constant for every fixed $\boldsymbol{n}=(n, n)$ with $n=10,50,100$. The relative errors decrease as $n$ increases, in accordance with Theorem 3.2. The convergence speed is not uniform: one of the reasons is that we are using a simple linear approximation of $f^{\dagger}$. In the last row we show relative errors between the gap of the biggest and the second-biggest eigenvalues and the gap between the maximum and second-maximum values of a uniform sampling of $f^{\dagger}$, confirming the prediction of Corollary 6.2. In particular, $d_{\boldsymbol{n}}\left(\lambda_{d_{\boldsymbol{n}}}^{(\boldsymbol{n})}-\lambda_{d_{\boldsymbol{n}^{-1}}}^{(\boldsymbol{n})}\right) \rightarrow\left(f^{\dagger}\right)^{\prime}(1) \in(0, \infty)$.

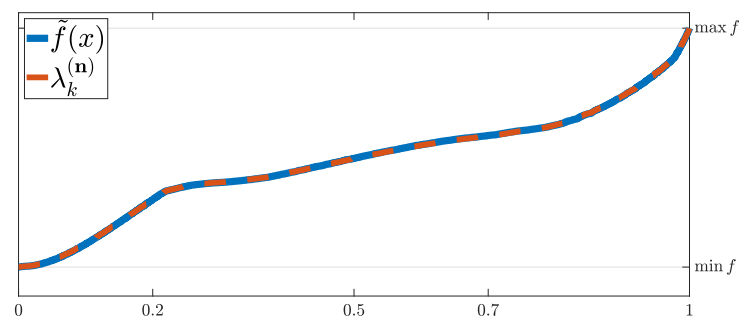

Figure 5. It is possible to observe the validity of Theorem 3.2, comparing the distribution of an approximation of the monotone rearrangement $f^{\dagger}$, for $x \in[0,1]$, and the distribution of $\lambda_{k}^{(\boldsymbol{n})}$, for $k(\boldsymbol{n}) / d_{\boldsymbol{n}} \in[0,1]$ and $\boldsymbol{n}=(60,60)$, i.e., $d_{\boldsymbol{n}}=36 \cdot 10^{2}$.

The map $\theta \mapsto 4 \boldsymbol{\theta}-(k-1) \pi$ is a diffeomorphism between $I_{k}$ and $[0, \pi]$, and the eigenvalues functions are ordered by magnitude, namely, $\lambda_{k}\left(\boldsymbol{f}_{k}(\theta)\right)$ is the $k$-th eigenvalue function of the matrix (14) over $\theta \in[0, \pi]$. In particular, in this case it holds that

$$
\begin{aligned}
\max _{\theta \in I_{k}} \lambda_{k}\left(\boldsymbol{f}_{k}(\theta)\right)=\max _{\theta \in[0, \pi]} \lambda_{k}(\boldsymbol{f}(\theta)) & <\min _{\theta \in[0, \pi]} \lambda_{k+1}(\boldsymbol{f}(\theta)) \\
& =\min _{\theta \in I_{k}} \lambda_{k+1}\left(\boldsymbol{f}_{k+1}(\theta)\right), \quad \text { for } k=1,2,3,
\end{aligned}
$$

and $\max _{\theta \in I_{4}} \lambda_{4}\left(\boldsymbol{f}_{4}(\theta)\right)=f^{\dagger}(1)>0$. In Figure 6 and Table 3 it is possible to check numerically the validity of Theorem 3.2 and Corollary 6.3. 


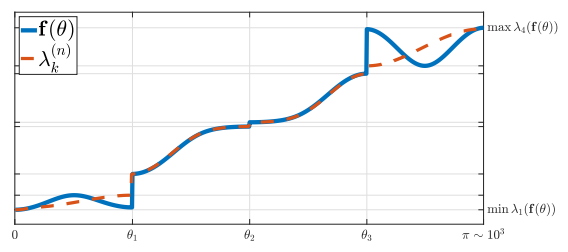

(A) Comparison between the distributions of $\boldsymbol{f}$ and $\lambda_{k}^{(n)}$

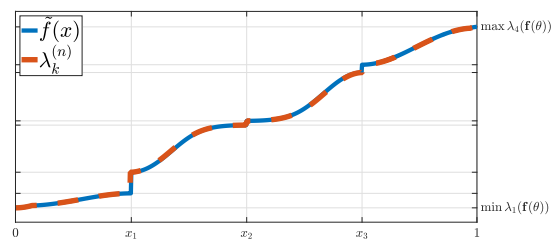

(B) Comparison between the distributions of $f^{\dagger}$ and $\lambda_{k}^{(n)}$

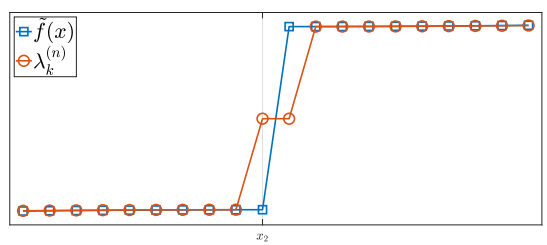

(C) Maginification of distribution graphs of $f^{\dagger}$ and $\lambda_{k}^{(n)}$ in a neighborhood of $x_{2}$.

Figure 6. In Figure 6a we compare the distributions of $f(\theta)$ from equation (15), for $\theta \in[0, \pi]$, and of the eigenvalues $\lambda_{k}^{(n)}$ of the adjacency matrix $W_{n}^{G}$, for $k=1, \ldots, 10^{3}$. We have that $\theta_{1}=\pi / 4$, $\theta_{2}=\pi / 2, \theta_{3}=3 \pi / 4$ are points of discontinuity since the inequalities in (16) are strict. With the notations of Definition 3.1, notice that in this example $R_{f}$ is the union of four disjoint intervals and that $\lambda_{k}^{(n)} \in\left[\min R_{f}, \max R_{f}\right]=\left[\min _{[0, \pi]} \lambda_{1}(\boldsymbol{f}(\theta)), \max _{[0, \pi]} \lambda_{4}(\boldsymbol{f}(\theta))\right]$. In Figure $4 \mathrm{~b}$, instead, it is possible to observe the validity of Theorem 3.2 , comparing the distribution of an approximation of the monotone rearrangement $f^{\dagger}$, for $x \in[0,1]$, and the distribution of $\lambda_{k}^{(n)}$, for $k(n) /(4 n) \in[0,1]$ and $4 n=10^{3}$. The points of discontinuity are now located at $x_{1}=1 / 4, x_{2}=1 / 2, x_{3}=3 / 4$. Finally, in figure $6 \mathrm{c}$ we zoom in a neighborhood of the discontinuity point $x_{2}$ : it is possible to observe the presence of two outliers which do not belong to $R_{f}$. This is not in contradiction with Theorem 3.2 since by Corollary 3.3 it admits at most a number of $o(4 n)$ of outliers.

\section{Applications to PDEs approximation}

The section is divided into three parts where we show that the approximation of a model differential problem by three celebrated approximation techniques leads to sequences of matrices that fall in the theory developed in the previous sections. 


\begin{tabular}{|c|c|c|c|c|}
\hline & \multicolumn{3}{|c|}{ relative errors } \\
\hline & & $4 n=10^{2}$ & $4 n=10^{3}$ & $4 n=5 \cdot 10^{3}$ \\
\hline \multirow{4}{*}{$\frac{k(n)}{4 n}$} & 0.1 & $5.2984 \mathrm{e}-04$ & $3.8322 \mathrm{e}-05$ & $1.0184 \mathrm{e}-05$ \\
\hline & 0.4 & 0.0285 & 0.0029 & $5.7782 \mathrm{e}-04$ \\
\hline & 0.8 & 0.0074 & $6.2919 \mathrm{e}-04$ & $1.2811 \mathrm{e}-04$ \\
\hline & 1 & $4.5189 \mathrm{e}-06$ & $4.6087 \mathrm{e}-09$ & $3.6928 \mathrm{e}-11$ \\
\hline \multicolumn{2}{|c|}{$4 n\left[\lambda_{4 n}^{(n)}-\lambda_{4 n-1}^{(n)}\right]$} & 3.2287 & 0.3253 & 0.0651 \\
\hline
\end{tabular}

TABLE 3. The first four rows present the relative errors between the eigenvalue $\lambda_{k(n)}^{(n)}$ and the sampling of the monotone rearrangement $f^{\dagger}\left(\frac{k(n)}{4 n}\right)$, i.e.: $\left|\frac{\lambda_{k(n)}^{(n)}}{f^{\dagger}(k(n) / 4 n)}-1\right|$. The index $k(n)$ of the eigenvalue $\lambda_{k(n)}^{(n)}$ is chosen such that $k(n) /(4 n)$ is constant for every fixed $4 n=10^{2}, 10^{3}, 5 \cdot 10^{3}$. As it can be seen, the relative errors decrease as $n$ increases, in accordance with Theorem 3.2. The convergence speed is not uniform: one of the reasons is that we are using a linear approximation of $f^{\dagger}$ instead of $f^{\dagger}$ itself. In the last row we show the computation of the gap between the biggest and the second-biggest eigenvalues, confirming the prediction of Corollary 6.3.

\subsection{Approximations of PDEs vs sequences of weighted d-level grid graphs: FD}

As a first example we consider the discretization of a self-adjoint operator $\mathcal{L}$ with (homogeneous) Dirichlet boundary conditions (BCs) on the disk $B_{1 / 2} \subset[0,1]^{2}$ by an equispaced Finite Difference (FD) approximation with $(4 m+1)$-points. That is, our model operator with Dirichlet BCs is given by

$$
\begin{aligned}
& \mathcal{L}: W_{0}^{1,2}\left(B_{\frac{1}{2}}\right) \rightarrow \mathrm{L}^{2}\left(B_{\frac{1}{2}}\right), \\
& \mathcal{L}[u](x, y):=-\operatorname{div}[p(x, y) \nabla u(x, y)]+q(x, y) u(x, y) \quad(x, y) \in B_{\frac{1}{2}},
\end{aligned}
$$

where

$$
B_{\frac{1}{2}}=\left\{(x, y) \in \mathbb{R}^{2}: 4\left(x-\frac{1}{2}\right)^{2}+4\left(y-\frac{1}{2}\right)^{2}<1\right\} .
$$

Fixing the diffusion term $p(x, y)=1+(x-1 / 2)^{2}+(y-1 / 2)^{2}$ and the potential term $q(x, y)=\mathrm{e}^{x y}$, then the operator $\mathcal{L}$ is self-adjoint and has purely point spectrum, see [13].

Now, if we fix $m=1, n \in \mathbb{N}$ and $i, j \in \mathbb{Z}$, then the uniform second-order 5-point FD approximation of the (negative) Laplacian operator (i.e., $\left.\Delta[\cdot]=-\left(\partial_{x^{2}}^{2}+\partial_{y^{2}}^{2}\right)[\cdot]\right)$ is given by

$$
\begin{aligned}
\Delta[u]\left(x_{i}, y_{j}\right) \approx & h^{-2}\left(-u\left(x_{i}, y_{j-1}\right)-u\left(x_{i-1}, y_{j}\right)+4 u\left(x_{i}, y_{j}\right)-u\left(x_{i+1}, y_{j}\right)\right. \\
& \left.-u\left(x_{i}, y_{j+1}\right)\right)
\end{aligned}
$$


for every $u \in C^{\infty}\left(\mathbb{R}^{2}\right)$, where $h=(n+1)^{-1}, x_{i}=\frac{i}{n+1}, y_{j}=\frac{j}{n+1}$. The same approximation applies for every $u \in C^{\infty}\left(B_{1 / 2}\right)$ and $i, j, n$ such that $\left\{\left(x_{i}, y_{j}\right),\left(x_{i \pm 1}, y_{j \pm 1}\right)\right\} \subset$ $B_{1 / 2}$. Notice that the weight of the central point $u\left(x_{i}, y_{j}\right)$ is the sum of all the other weights, changed of sign.

Let us consider the 2-level Toeplitz graph $T_{\boldsymbol{n}}\langle\{[1,0], 1\},\{[0,1], 1\}\rangle$ with $\boldsymbol{n}=$ $(n, n)$ as in Definition 4.2 and immerse it in $B_{1 / 2}$ as in Definition 5.2, i.e., $T_{\boldsymbol{n}}^{B_{1 / 2}}\left\langle\left\{[1,0], w^{p}\right\},\left\{[0,1], w^{p}\right\}\right\rangle$ such that

$$
\begin{aligned}
& V_{\boldsymbol{n}}=\left\{\left(x_{i}, y_{j}\right) \in[0,1]^{2}:\left(x_{i}, y_{j}\right) \in B_{\frac{1}{2}}\right\} \\
& w^{p}\left(\left(x_{i}, y_{j}\right),\left(x_{r}, y_{s}\right)\right):=1+\left(\frac{x_{i}+x_{r}}{2}-\frac{1}{2}\right)^{2}+\left(\frac{y_{j}+y_{s}}{2}-\frac{1}{2}\right)^{2}
\end{aligned}
$$

if $(|i-r|,|j-s|) \in\{(1,0),(0,1)\}$. Extend now continuously the diffusion term $p(x, y)$ outside $B_{1 / 2}$, that is,

$$
\bar{p}(x, y)= \begin{cases}\frac{5}{4} & \text { if }(x, y) \in \mathbb{R}^{2} \backslash B_{\frac{1}{2}} \\ p(x, y) & \text { if }(x, y) \in B_{\frac{1}{2}}\end{cases}
$$

and define the graph

$$
G_{\boldsymbol{n}}=\left(T_{\boldsymbol{n}}^{B_{1 / 2}}\left\langle\left\{[1,0], w^{p}\right\},\left\{[0,1], w^{p}\right\}\right\rangle, \kappa\right),
$$

as a sub-graph of

$$
\begin{aligned}
\bar{G}_{\boldsymbol{n}}=\left(T_{\boldsymbol{n}}\left\langle\left\{[1,0], w^{\bar{p}}\right\},\left\{[0,1], w^{\bar{p}}\right\}\right\rangle, \bar{\kappa}\right) \text { where } & \bar{V}_{\boldsymbol{n}}=\left\{\left(x_{i}, y_{j}\right) \in[0,1]^{2}\right\}, \\
& \bar{p} \text { as in }(19), \\
& \bar{\kappa}\left(x_{i}, y_{j}\right)=h^{2} q\left(x_{i}, y_{j}\right) .
\end{aligned}
$$

Namely, the host graph $\bar{G}_{\boldsymbol{n}}$ is the 2-level grid graph on $[0,1]^{2}$ obtained by extending continuously the diffusivity function $p$ to $[0,1]^{2}$ and adding a nontrivial potential term $\bar{\kappa}$ which naturally depends on $q$. On the other hand, the potential term $k$ of the sub-graph $G_{\boldsymbol{n}}$ describes the edge deficiency of nodes in $G_{\boldsymbol{n}}$ compared to the same nodes in $\bar{G}_{\boldsymbol{n}}$,

$$
\begin{aligned}
\kappa\left(x_{i}, y_{j}\right)=h^{2} q\left(x_{i}, y_{j}\right)+ & \sum_{\substack{\left(x_{r}, y_{s}\right) \sim\left(x_{i}, y_{j}\right) \\
\left(x_{r}, y_{s}\right) \in \bar{V}_{\boldsymbol{n}} \backslash V_{\boldsymbol{n}}}} w^{\bar{p}}\left(\left(x_{r}, y_{s}\right),\left(x_{i}, x_{j}\right)\right), \\
\left|\kappa\left(x_{i}, y_{j}\right)-\bar{\kappa}\left(x_{i}, y_{j}\right)\right|= & \sum_{\substack{\left(x_{r}, y_{s}\right) \sim\left(x_{i}, y_{j}\right) \\
\left(x_{r}, y_{s}\right) \in \bar{V}_{\boldsymbol{n}} \backslash V_{\boldsymbol{n}}}} w^{\bar{p}}\left(\left(x_{r}, y_{s}\right),\left(x_{i}, x_{j}\right)\right),
\end{aligned}
$$

see [33, p. 197]. It is easy to check that the boundary points $\left(x_{i}, x_{j}\right) \in \partial V_{\boldsymbol{n}}$ are connected at most with two points of $\bar{V}_{\boldsymbol{n}} \backslash V_{\boldsymbol{n}}$, therefore the potential term $\kappa$ can be 


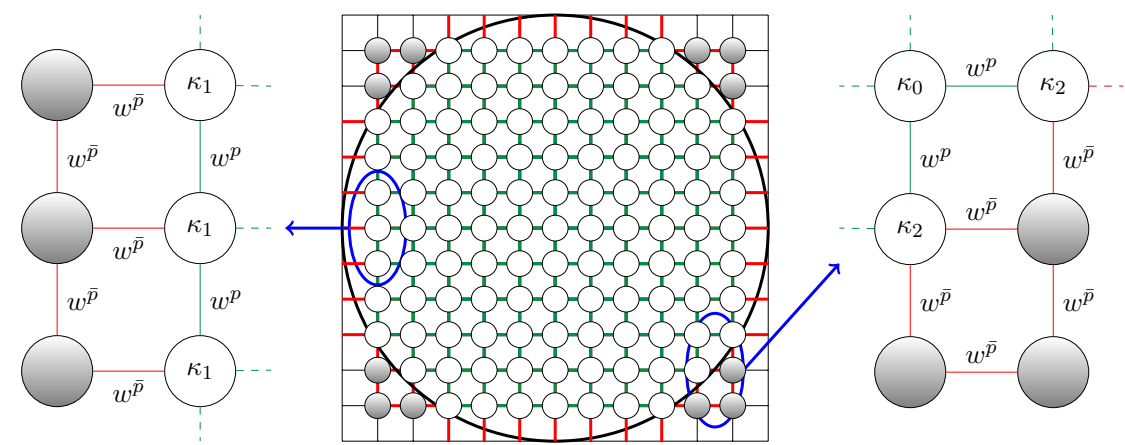

FIGURE 7. Immersion of a 2-level grid graph inside the disk $B_{1 / 2} \subset$ $[0,1]^{2}$. The white nodes are the nodes of $V_{\boldsymbol{n}}$ while the gray nodes belong to $\bar{V}_{\boldsymbol{n}} \backslash V_{\boldsymbol{n}}$. The potential term $\bar{\kappa}$ of the host graph $\bar{G}_{n}$ is determined only by the potential term $q$ from (17) while the potential term $\kappa$ of the sub-graph $G_{\boldsymbol{n}}$ is influenced by the nodes in $\bar{V}_{\boldsymbol{n}} \backslash V_{\boldsymbol{n}}$ on the boundary set $\partial V_{\boldsymbol{n}}$. This influence is due to the presence of Dirichlet BCs in (18). The green connections represent the weighted edges whose end-nodes are both interior nodes of $V_{\boldsymbol{n}}$, while the red connections represent the weighted edges which have at least one end-node that belongs to $\bar{V}_{\boldsymbol{n}} \backslash V_{\boldsymbol{n}}$. The potential term $\kappa$ sums the weight of a red edge to every of its end-nodes which belong to $V_{\boldsymbol{n}}$.

split into three terms

$$
\kappa\left(x_{i}, y_{j}\right)= \begin{cases}\kappa_{0}\left(x_{i}, y_{j}\right)=h^{2} q\left(x_{i}, y_{j}\right) & \text { if }\left(x_{i}, y_{j}\right) \in \stackrel{\circ}{V}_{\boldsymbol{n}}, \\ \kappa_{1}\left(x_{i}, y_{j}\right)=h^{2} q\left(x_{i}, y_{j}\right)+\operatorname{deg}\left(\left(x_{i}, y_{j}\right)\right), & \text { if } \exists ! \text { one neighbor } \\ \kappa_{2}\left(x_{i}, y_{j}\right)=h^{2} q\left(x_{i}, y_{j}\right)+\operatorname{deg}\left(\left(x_{i}, y_{j}\right)\right), & \text { in } \bar{V}_{\boldsymbol{n}} \backslash V_{\boldsymbol{n}}, \\ & \text { if } \exists ! \text { two neighbors } \\ & \text { in } \bar{V}_{\boldsymbol{n}} \backslash V_{\boldsymbol{n}} .\end{cases}
$$

See Figure 7.

The given graph-Laplacian $\Delta_{G_{n}}$ approximates the weighted operator $h^{2} \mathcal{L}$. Moreover, by Corollary 5.3 it holds that

$$
\left\{\Delta_{G_{n}}\right\} \sim_{\lambda} \mathfrak{f}\left(x, y, \theta_{1}, \theta_{2}\right), \quad\left(x, y, \theta_{1}, \theta_{2}\right) \in B_{\frac{1}{2}} \times[-\pi, \pi]^{2}
$$

where

$$
\begin{aligned}
\mathfrak{f}\left(x, y, \theta_{1}, \theta_{2}\right) & =p(x, y)\left(4-f\left(\theta_{1}, \theta_{2}\right)\right) \\
& =\left[1+(x-1 / 2)^{2}+(y-1 / 2)^{2}\right]\left(4-2 \cos \left(\theta_{1}\right)-2 \cos \left(\theta_{2}\right)\right) .
\end{aligned}
$$

By the symmetry of $\mathfrak{f}$ over $[-\pi, \pi]^{2}$ we can restrict it to $B_{1 / 2} \times[0, \pi]^{2}$ without affecting the validity of the identity (3). If we consider now the monotone rearrangement $\mathfrak{f}^{\dagger}:[0,1] \rightarrow[0,10]$ of the symbol $\mathfrak{f}$ as in Definition 3.1 , then we can see from Table 4 


\begin{tabular}{|c|c|ccc|}
\cline { 3 - 5 } \multicolumn{2}{c|}{} & \multicolumn{3}{c|}{ relative errors } \\
\cline { 3 - 5 } \multicolumn{2}{c|}{} & $n=10$ & $n=50$ & $n=80$ \\
\hline \multirow{3}{*}{$\frac{k(\boldsymbol{n})}{d_{\boldsymbol{n}}}$} & 0.1 & 0.0788 & 0.0094 & 0.0020 \\
\cline { 2 - 5 } & 0.5 & 0.0055 & $3.3995 \mathrm{e}-04$ & $1.2565 \mathrm{e}-04$ \\
\cline { 2 - 5 } & 0.8 & 0.0100 & $7.2173 \mathrm{e}-04$ & $3.9353 \mathrm{e}-05$ \\
\hline
\end{tabular}

TABLE 4. Relative errors between the eigenvalue $\lambda_{k(\boldsymbol{n})}^{(\boldsymbol{n})}$ and the sampling of the monotone rearrangement $\mathfrak{f}^{\dagger}\left(\frac{k(\boldsymbol{n})}{d_{\boldsymbol{n}}}\right)$, i.e.: $\left|\frac{\lambda_{k(\boldsymbol{n})}^{(\boldsymbol{n})}}{\mathfrak{f}^{\dagger}\left(k(\boldsymbol{n}) / d_{\boldsymbol{n}}\right)}-1\right|$. The index $k(\boldsymbol{n})$ of the eigenvalue $\lambda_{k(\boldsymbol{n})}^{(\boldsymbol{n})}$ is chosen such that $k(\boldsymbol{n}) / d_{\boldsymbol{n}}$ is constant for every fixed $n=10,50,80$. As it can be seen, the relative errors decrease as $n$ increases, in accordance with Theorem 3.2. The convergence to zero is not uniform and slower in some subintervals.

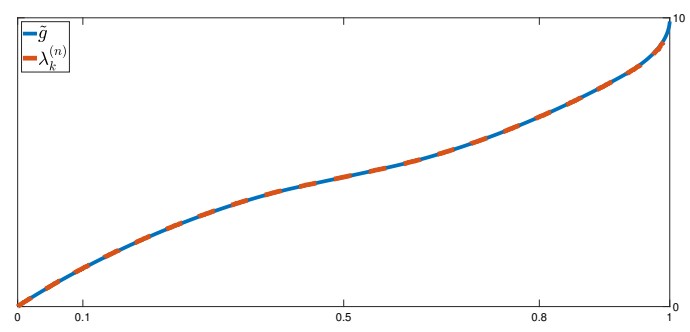

Figure 8. Plots of the monotone rearrangement $\mathfrak{f}^{\dagger}$ (blue-continuous line) and the eigenvalues $\left\{\lambda_{k}^{(\boldsymbol{n})}\right\}$ (orange-dotted line), for $n=80$, of the graph-Laplacian $\Delta_{G_{n}}$ associated with the graph $G_{\boldsymbol{n}}$ defined in (20). In this case we have that $d_{\boldsymbol{n}}=5140<80^{2}$.

and Figure 8 that

$$
\lim _{n \rightarrow \infty} \lambda_{k(\boldsymbol{n})}^{(\boldsymbol{n})} \rightarrow \mathfrak{f}^{\dagger}\left(\frac{k(\boldsymbol{n})}{d_{\boldsymbol{n}}}\right)
$$

for any index sequence $\{k(\boldsymbol{n})\}, 1 \leq k(\boldsymbol{n}) \leq d_{\boldsymbol{n}}$, such that $\frac{k(\boldsymbol{n})}{d_{\boldsymbol{n}}} \rightarrow x \in[0,1]$ as $\boldsymbol{n} \rightarrow \infty$, where $d_{\boldsymbol{n}}$ is the dimension of the graph-Laplacian $\Delta_{G_{\boldsymbol{n}}}$. We want to stress out that $d_{\boldsymbol{n}}<n^{2}$ since $V_{\boldsymbol{n}} \subsetneq \bar{V}_{\boldsymbol{n}}$, but clearly it holds that $d_{\boldsymbol{n}} \rightarrow \infty$ as $n \rightarrow \infty$, and the Hausdorff distance between the node set $V_{\boldsymbol{n}}$ and the disk $B_{1 / 2}$ is going to zero.

Since $\mathfrak{f}^{\dagger}$ does not posses an easy analytical expression to calculate, it has been approximated by an equispaced sampling of $\mathfrak{f}$ over $B_{1 / 2} \times[0, \pi]^{2}$ by $d_{\boldsymbol{n}}$-points and then rearranging it in non-decreasing order. The approximation converges to $\mathfrak{f}^{\dagger}$ as $n \rightarrow \infty$, see for example [40]. Finally, see Remark 3.1.

For other applications of the GLT techniques for approximation of partial differential operators see [3]. 


\subsection{Approximations of PDEs vs sequences of weighted diamond graphs: FEM}

Consider the model boundary value problem

$$
\begin{cases}-\Delta u=g & \text { in } \Omega, \\ u=0 & \text { on } \partial \Omega,\end{cases}
$$

where $\Omega=(0,1)$ and $g \in L^{2}(\Omega)$. We approximate (21) by using the quadratic FEM over the uniform mesh with stepsize $\frac{1}{n+1}$, defined on the knot sequence

$$
\left\{\frac{1}{n+1}, \frac{1}{n+1}, \frac{2}{n+1}, \frac{2}{n+1}, \ldots, \frac{n}{n+1}, \frac{n}{n+1}\right\} .
$$

The Finite Element basis is chosen as the quadratic $C^{0} \mathrm{~B}$-spline basis over the knot sequence.

Proceeding as in [25], we trace the problem back to solving a linear system whose stiffness matrix reads as follows:

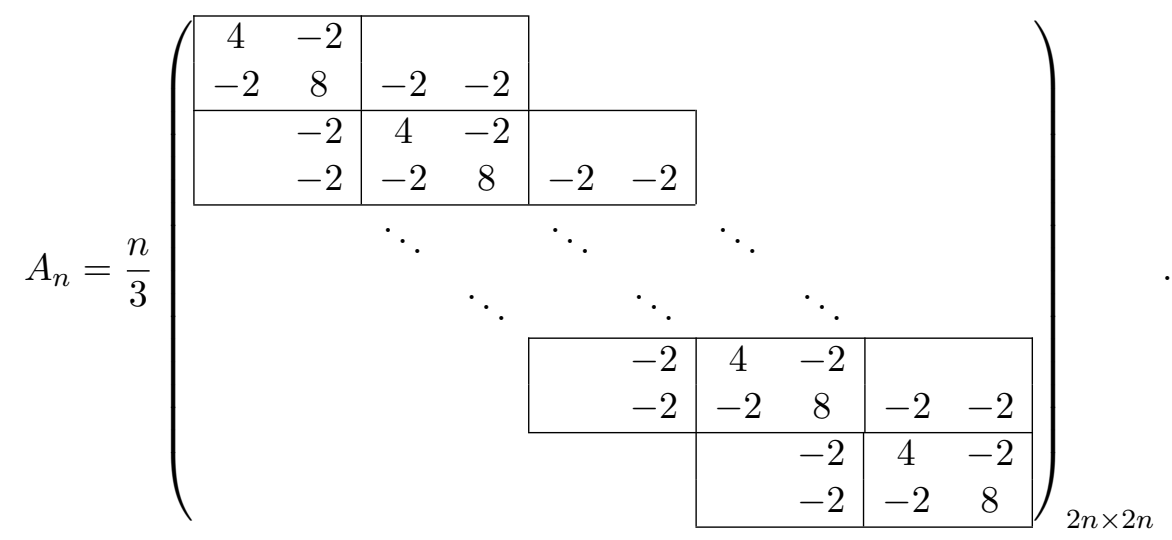

We note that $\frac{1}{n} A_{n}$ can be seen as the graph-Laplacian of a 1-level diamond Toeplitz graph with nonzero potential term. Namely, according to Definition 2.2, we have that

$$
\frac{1}{n} A_{n}=K_{n}-W_{n, 2}^{G}
$$

where $K_{n}$ is the diagonal matrix given by

$$
\left(K_{n}\right)_{i i}= \begin{cases}\frac{4}{3} & \text { if } i \text { is even } \\ \frac{8}{3} & \text { otherwise }\end{cases}
$$

and $W_{n, 2}^{G}$ is the adjacency matrix of the 1-level diamond Toeplitz graph $T_{3}^{G}\left\langle\left(1, L_{1}\right)\right\rangle$, with

$$
G=\left(\begin{array}{ll}
0 & 2 \\
2 & 0
\end{array}\right)
$$

and

$$
L_{1}=\left(\begin{array}{cc}
0 & 0 \\
2 & 2
\end{array}\right)
$$


Combining now Proposition 4.4 and Corollary 5.3 we get that $\frac{1}{n} A_{n}$ has asymptotic spectral distribution with symbol function $f:[-\pi, \pi] \rightarrow \mathbb{C}^{2 \times 2}$ given by

$$
\begin{aligned}
\boldsymbol{f}(\theta) & =\frac{1}{3}\left\{\left(\begin{array}{cc}
4 & -2 \\
-2 & 8
\end{array}\right)+\left(\begin{array}{cc}
0 & -2 \\
0 & -2
\end{array}\right) \mathrm{e}^{\mathrm{i} \theta}+\left(\begin{array}{cc}
0 & 0 \\
-2 & -2
\end{array}\right) \mathrm{e}^{-\mathrm{i} \theta}\right\} \\
& =\frac{1}{3}\left\{\left(\begin{array}{cc}
4 & -2 \\
-2 & 8
\end{array}\right)+\left(\begin{array}{cc}
0 & -2 \\
-2 & -4
\end{array}\right) \cos (\theta)+\left(\begin{array}{cc}
0 & -2 \\
2 & 0
\end{array}\right) \mathrm{i} \sin (\theta)\right\} .
\end{aligned}
$$

It is possible to study the multi-dimensional case of the problem in the example above using the fact that for every $\boldsymbol{m}, \boldsymbol{s} \in \mathbb{N}^{d}$ there exists a permutation matrix $\Gamma_{\boldsymbol{m}, \boldsymbol{s}}$ of dimension $\prod_{j=1}^{d} m_{j} s_{j}$ such that

$$
T_{m_{1}}\left(\boldsymbol{p}_{1}\right) \otimes T_{m_{2}}\left(\boldsymbol{p}_{2}\right) \cdots \otimes T_{m_{d}}\left(\boldsymbol{p}_{d}\right)=\Gamma_{\boldsymbol{m}, \boldsymbol{s}}\left[T_{\boldsymbol{m}}\left(\boldsymbol{p}_{1}\left(\theta_{1}\right) \otimes \cdots \otimes \boldsymbol{p}\left(\theta_{d}\right)\right)\right] \Gamma_{\boldsymbol{m}, \boldsymbol{s}}^{*}
$$

for any choice of trigonometric polynomials $\boldsymbol{p}:[-\pi, \pi] \rightarrow \mathbb{C}^{s_{j} \times s_{j}}, j=1, \ldots, d$, as stated in Lemma 4 of [25], where $\otimes$ denotes the usual tensor product. In the $d$ dimensional case (see, again, [25] and references therein), the discretizing matrix is given by

$$
A_{\boldsymbol{n}}=\sum_{k=1}^{d}\left(\bigotimes_{r=1}^{k-1} \frac{1}{n_{r}} M_{n_{r}}\right) \otimes A_{n_{k}} \otimes\left(\bigotimes_{r=k+1}^{d} \frac{1}{n_{r}} M_{n_{r}}\right),
$$

where $A_{n}$ is defined as above and $M_{n}$ is a $2 n \times 2 n$ matrix with the same block Toeplitz structure as $A_{n}$ and, hence, an analogous symbol function, which we denote by $\boldsymbol{h}$. Assuming now that the multi-index $\boldsymbol{n}=\boldsymbol{\nu} n=\left(\nu_{1} n, \nu_{2} n, \ldots, \nu_{d} n\right)$ for a fixed

$$
\boldsymbol{\nu} \in \mathbb{Q}_{>0}^{d}:=\left\{\left(\nu_{1}, \ldots, \nu_{d}\right) \in \mathbb{Q}: \nu_{1}, \ldots, \nu_{d}>0\right\},
$$

it is immediate to see by the considerations above that $n^{d-2} A_{\boldsymbol{n}}$ is the linear combination of graph-Laplacians of $d$-level diamond Toeplitz graphs with spectral distribution given by the following symbol function $\mathfrak{f}:[-\pi, \pi]^{d} \rightarrow \mathbb{C}^{2^{d} \times 2^{d}}$,

$$
\mathfrak{f}(\boldsymbol{\theta})=\sum_{k=1}^{d} c_{k}(\boldsymbol{\nu})\left(\bigotimes_{r=1}^{k-1} \boldsymbol{h}\left(\theta_{r}\right)\right) \otimes \boldsymbol{f}\left(\theta_{k}\right) \otimes\left(\bigotimes_{r=k+1}^{d} \boldsymbol{h}\left(\theta_{r}\right)\right),
$$

with

$$
c_{k}(\boldsymbol{\nu})=\frac{\nu_{k}}{\nu_{1} \cdots \nu_{k-1} \nu_{k+1} \cdots \nu_{d}}, k=1, \ldots, d .
$$

\subsection{Approximations of PDEs vs sequences of weighted d-level graphs: IgA approach}

In this subsection we consider the approximation of the same differential equation (21) by using the Galerkin B-splines approximation of degree $\nu$ in every direction: while the approximation of standard $\mathbb{Q}_{\nu}$ Lagrangian FEM considered in Subsection 7.2 leads to a symbol which is a linear trigonometric polynomial in every variable, but $\mathbb{C}^{\nu^{d} \times \nu^{d}}$ Hermitian matrix-valued, here the symbol is scalar-valued, but the degree of the trigonometric polynomial is much higher. For example, by means of cubic $C^{2}$ B-spline discretization the (normalized) stiffness matrix is given by 


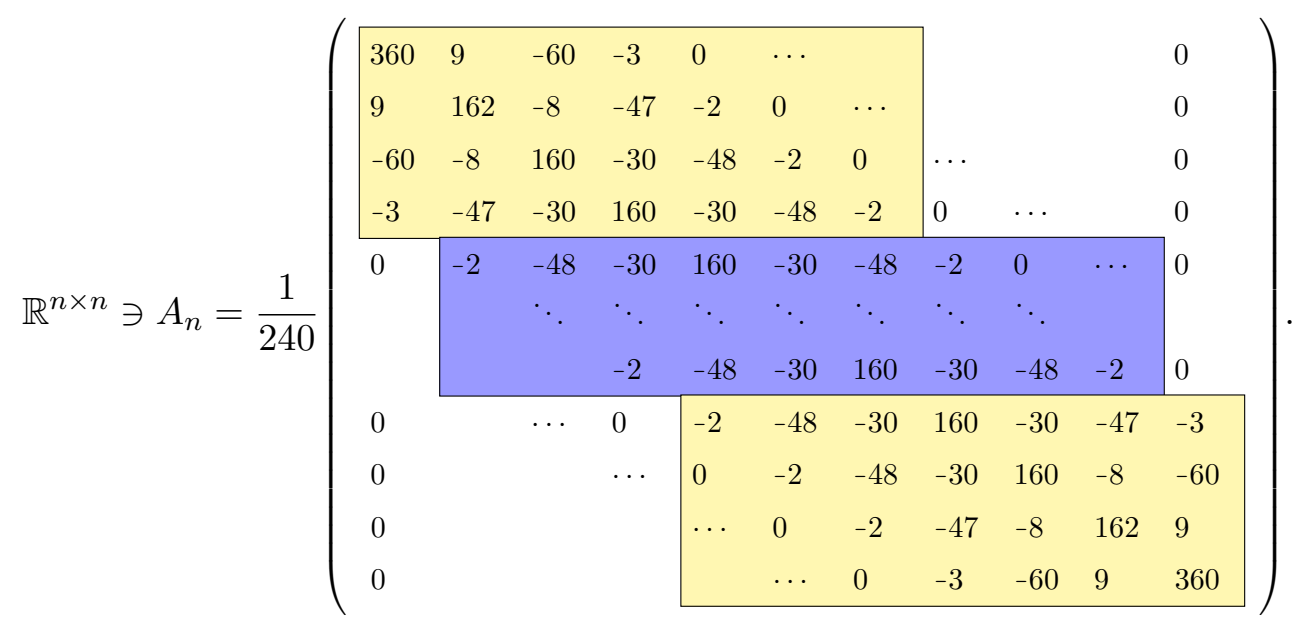

Observe that the principal $\mathbb{R}^{(n-4) \times(n-4)}$-submatrix (highlighted in blue) is an exact symmetric Toeplitz matrix while globally $A_{n}$ is not Toeplitz due to the presence of perturbations near the boundary points (highlighted in yellow). This behavior is influenced by the presence of BCs and the specific choice for the test-functions (B-spline with $C^{3}$ local regularity and $C^{2}$ global regularity). For those reasons, $A_{n}$ can not be representative of the graph-Laplacian of a graph in the form $G_{n}=$ $\left(T_{n}\left\langle\left(t_{1}, w_{1}\right), \ldots,\left(t_{m}, w_{m}\right)\right\rangle, \kappa\right)$, even if it is clearly the graph-Laplacian for another kind of graph $G_{n}$ which does not own globally the symmetries of the graphs studied in Sections 4 and 5. Nevertheless, since the perturbations are local, it happens that $\left\{A_{n}\right\}_{n} \sim_{\lambda} f(\theta)$ where $f$ is the same symbol function of the graph-Laplacian of

$$
G_{n}=\left(T_{n}\left\langle\left(1, \frac{30}{240}\right),\left(1, \frac{48}{240}\right),\left(1, \frac{2}{240}\right)\right\rangle, \kappa\right), \quad \kappa \equiv 0,
$$

namely, $f(\theta)=\frac{160}{240}-\frac{60}{240} \cos (\theta)-\frac{96}{240} \cos (2 \theta)-\frac{4}{240} \cos (3 \theta)$. For a complete treatment of the study of the eigenvalue distribution for Galerkin B-splines approximations we refer to [27], where many examples are provided along the exposure.

There is another quite important difference between this case and the case of Subsection 7.1. In the FD case, the nodes of the graph were representative of the physical domain while in this case, even if the node set can be immersed in $[0,1]$, the nodes represent the base functions of the test-functions set. That said, given $A_{n}$, it is of interest to calculate the corresponding weight function $w$ on the node set of the physical domain: this approach could lead some insight about the problem of the presence of a fixed number of outliers in the spectrum of $A_{n}$, see [11, Chapter 5.1.2 p. 153].

\section{Conclusions and future work}

We have defined general classes of graph sequences having a grid geometry with a uniform local structure in a domain $\Omega \subset[0,1]^{d}, d \geq 1$. With the only weak 
requirement that $\Omega$ is Lebesgue measurable with boundary of zero Lebesgue measure, we have shown that the underlying sequences of adjacency matrices have a canonical eigenvalue distribution, in the Weyl sense, with a symbol $\mathfrak{f}$ being a trigonometric polynomial in the $d$ Fourier variables: as specific cases, we mention standard Toeplitz graphs, when $\Omega=[0,1]$, and $d$-level Toeplitz graphs when $\Omega=[0,1]^{d}$, but also matrices coming from the approximation of differential operators by local techniques, including Finite Differences, Finite Elements, Isogeometric Analysis etc. In such a case we considered block structures and weighted graphs, from the perspective of GLT sequences, where the tools taken from the latter field have resulted crucial for deducing all the asymptotic spectral results. In particular, the knowledge of the symbol and of basic analytical features have been employed for deducing a lot of information on the eigenvalue structure, including precise asymptotics on the gaps between the largest eigenvalues, specific preconditioners and projectors for the fast solution of the underlying (large) linear systems (see [1]).

Many open problems remain, ranging from a deeper analysis of the matrixsequences arising from different families of Finite Element approximations of multidimensional differential problems to the study of the convergence features of the ordered asymptotic spectra, to more computational techniques for the related large linear systems, to the rearrangement of the corresponding symbol (see also the study and discussions in $[2,27])$.

Acknowledgements. We thank the Editors and the anonymous referees for their careful reading and for their pertinent suggestions that helped us to improve the quality of the paper. We also thank INdAM - GNCS for the support in conducting our researches.

Funding. Open access funding provided by Università degli Studi dell'Insubria within the CRUI-CARE Agreement.

Open Access. This article is licensed under a Creative Commons Attribution 4.0 International License, which permits use, sharing, adaptation, distribution and reproduction in any medium or format, as long as you give appropriate credit to the original author(s) and the source, provide a link to the Creative Commons licence, and indicate if changes were made. The images or other third party material in this article are included in the article's Creative Commons licence, unless indicated otherwise in a credit line to the material. If material is not included in the article's Creative Commons licence and your intended use is not permitted by statutory regulation or exceeds the permitted use, you will need to obtain permission directly from the copyright holder. To view a copy of this licence, visit http://creativecommons.org/licenses/by/4.0/.

Publisher's Note. Springer Nature remains neutral with regard to jurisdictional claims in published maps and institutional affiliations. 


\section{References}

[1] A. Adriani, D. Bianchi, P. Ferrari, S. Serra-Capizzano, M. Usman, Asymptotic spectra of large (grid) graphs with a uniform local structure (part II): numerical applications, submitted (2020).

[2] D. Bianchi, Analysis of the spectral symbol associated to discretization schemes of linear self-adjoint differential operators, submitted (2020): arXiv:2004.10058.

[3] D. Bianchi, S. Serra-Capizzano, Spectral analysis of finite-dimensional approximations of 1d waves in non-uniform grids, Calcolo 55 (2018), paper no. 47, 28pp.

[4] R. Bhatia, Matrix Analysis, Springer-Verlag, New York, 1997.

[5] A. Böttcher and B. Silbermann, Introduction to Large Truncated Toeplitz Matrices, Springer-Verlag, New York, 1999.

[6] A. E. Brouwe, W.H. Haemers, Spectra of graphs, Springer Science \& Business Media, New York, 2011.

[7] J. Bu, C. Chen, C. Wang, X. Wang, Y. Wu, Z.Yu, Friend recommendation with content spread enhancement in social networks, Inf. Sci. 309 (2015), pp. 102-118.

[8] D. Burago, S. Ivanov, Y. Kurylev, A graph discretization of the Laplace-Beltrami operator, J. Spectr. Theor. 4 no. 4 (2014), 675-715.

[9] K.L. Chung, A course in probability theory, Academic Press, Cambridge, 2001.

[10] P. Ciarlet, The Finite Element Method for Elliptic Problems, North Holland, Amsterdam, 1978.

[11] J.A. Cottrell, T.J.R. Hughes, Y. Bazilevs, Isogeometric analysis: toward integration of CAD and FEA, John Wiley \& Sons, Hoboken, 2009.

[12] D. Cvetkovic, M. Doob, H. Sachs, Spectra of Graphs, Academic Press, New York, 1979.

[13] E.B. DAvies, Spectral theory and differential operators, Cambridge University Press, 1996.

[14] F. Di Benedetto, G. Fiorentino, S. Serra-Capizzano, CG preconditioning for Toeplitz matrices, Comput. Math. with Appl. 25 no. 6 (1993), 35-45.

[15] B.E. Eichinger, An approach to distribution functions for Gaussian molecules, Macromolecules 10 no. 3 (1977), 671-675.

[16] E. Estrada, Path Laplacian matrices: Introduction and application to the analysis of consensus in networks, Linear Algebra Appl. 436 no. 9 (2012), 3373-3391.

[17] E. Estrada, E. Hameed, N. Hatano, M. Langer, Path Laplacian operators and superdiffusive processes on graphs, I. One-dimensional case, Linear Algebra Appl. $\mathbf{5 2 3}$ (2017), 307-334.

[18] E. Estrada, E. Hameed, M. Langer, A. Puchalska, Path Laplacian operators and superdiffusive processes on graphs, II. Two-dimensional lattice, Linear Algebra Appl. 555 (2018), 373-397 (2018).

[19] J.A. Fax, R.M. Murray, R. Olfati-Saber, Consensus and cooperation in networked multi-agent systems, Proc. IEEE 95 no. 1 (2007), 215-233.

[20] M. FiedLer, Algebraic connectivity of graphs, Czechoslov. Math. J. 23 no. 2 (1973), 298-305. 
[21] H. Galina, M. Syslo, Some applications of graph theory to the study of polymer configuration, Discret. Appl. Math. 19 (1988), 167-176.

[22] C. Garoni, M. Mazza, S. Serra-Capizzano, Block generalized locally Toeplitz sequences: from the theory to the applications, Axioms 7 no. 3 (2018), paper no. 49.

[23] C. Garoni and S. Serra-Capizzano, The theory of Generalized Locally Toeplitz sequences: theory and applications, vol. I, Springer Monographs in Mathematics, Springer, New York, 2017.

[24] C. Garoni and S. Serra-Capizzano, The theory of multilevel Generalized Locally Toeplitz sequences: theory and applications, vol. II, Springer Monographs in Mathematics, Springer, New York, 2018.

[25] C. Garoni, S. Serra-Capizzano, D. Sesana, Spectral analysis and spectral symbol of $d$-variate $\mathbb{Q}_{p}$ Lagrangian FEM stiffness matrices, SIAM J. Matrix Anal. Appl. 36 no. 3 (2015), 1100-1128.

[26] C. Garoni, S. Serra-Capizzano, D. Sesana, The Theory of Block Generalized Locally Toeplitz Sequences, Technical Report, no. 1, January 2018, Department of Information Technology, Uppsala University, http://www.it.uu.se/research/publications/reports/2018-001/

[27] C. Garoni, H. Speleers, S.-E. Ekstrøm, A. Reali, S. Serra-Capizzano, T.J.R. Hughes, Symbol-based analysis of finite element and isogeometric B-spline discretizations of eigenvalue problems: Exposition and review, Arch. Comput. Methods Eng. 26 (2019), 1639-1690.

[28] S. Hossein Ghorban, Toeplitz graph decomposition, Trans. Combinat. 1 no. 4 (2012), $35-41$.

[29] U. Grenander And G. Szegő, Toeplitz Forms and Their Applications, 2nd ed., Chelsea, New York, 1984.

[30] M. Kac, W.L. Murdoch, G. Szegö, On the eigenvalues of certain Hermitian forms, J. Rational Mech. Anal. 2 (1953), 767-800.

[31] T. Kato, Perturbation theory for linear operators, 2nd ed., Springer, Heidelberg, 1980.

[32] B. KAwOHL, Rearrangements and convexity of level sets in PDE, Springer, Heidelberg, 1985 .

[33] M. Keller And D. Lenz, Dirichlet forms and stochastic completeness of graphs and subgraphs, J. Reine Angew. Math. 666 (2012), 189-223.

[34] C. MaAs, Transportation in graphs and the admittance spectrum, Discret. Appl. Math. 16 no. 1 (1987), 31-49.

[35] B. Mohar, Y. Alavi, G. Chartrand, O.R. Oellermann, The Laplacian spectrum of graphs, Graph theory, combinatorics, and applications 2 no. 12 (1991), 871-898.

[36] S. Serra-Capizzano, Asymptotic results on the spectra of block Toeplitz preconditioned matrices, SIAM J. Matrix Anal. Appl. 20 no. 1 (1998), 31-44.

[37] S. Serra-Capizzano, Generalized locally Toeplitz sequences: spectral analysis and applications to discretized partial differential equations, Linear Algebra Appl. 366 (2003), 371-402.

[38] S. Serra-Capizzano, The GLT class as a generalized Fourier analysis and applications, Linear Algebra Appl. 419 no. 1 (2006), 180-233. 
[39] J.C. Strikwerda, Finite Difference Schemes and Partial Differential Equations, Chapman and Hall, International Thompson Publ., New York, 1989.

[40] G. TAlenti, Rearrangements of functions and partial differential equations, in: Nonlinear Diffusion Problems, Springer, Berlin, Heidelberg, 1986, pp. 153-178.

[41] P. TilLI, Locally Toeplitz matrices: spectral theory and applications, Linear Algebra Appl. 278 (1998), 91-120.

[42] P. TILli, A note on the spectral distribution of Toeplitz matrices, Linear Multilinear Algebra 45 (1998), 147-159.

[43] N. G. Trillos, M. Gerlach, M. Hein, D. Slepčev, Error estimates for spectral convergence of the graph Laplacian on random geometric graphs toward the Laplace-Beltrami operator, Found. Computh. Math. (2019), DOI: 10.1007/s10208-01909436-w.

[44] N. G. Trillos, D. Sanz-Alonso, Continuum limits of posteriors in graph Bayesian inverse problems, SIAM J. Math. Anal. 50 no. 4 (2018), 4020-4040.

[45] N. G. Trillos, D. SAnz-Alonso, R. Yang, Local Regularization of Noisy Point Clouds: Improved Global Geometric Estimates and Data Analysis, J. Mach. Learn. Res. 20 (2019), paper no. 136, 37pp.

[46] E. Tyrtyshnikov and N. Zamarashinin, Spectra of multilevel Toeplitz matrices: advanced theory via simple matrix relationships, Linear Algebra Appl. 270 (1998), 1527.

Andrea Adriani and Davide Bianchi

Department of Theoretical and Applied Sciences, University of Insubria

Via Valleggio 11, 22100 Como, Italy

e-mail: aadriani@uninsubria.it

d.bianchi9@uninsubria.it

Stefano Serra-Capizzano

Department of Humanities and Innovation, University of Insubria

Via Valleggio 11, 22100 Como, Italy

and

Department of Information Technology, Uppsala University, Uppsala, Sweden

e-mail: stefano.serrac@uninsubria.it, stefano.serra@it.uu.se

Received: June 2, 2020.

Accepted: July 26, 2020. 